\title{
Geometric quantization of symplectic maps and Witten's asymptotic conjecture
}

\author{
Louis IOOS
}

\begin{abstract}
We use the theory of Berezin-Toeplitz operators of Ma and Marinescu to study the spaces of holomorphic sections of a prequantizing line bundle over compact Kähler manifolds under deformations of the complex structure. We show that the parallel transport in the induced vector bundle over the deformation space behaves like a Toeplitz operator, and compute its first coefficient. We then use this result to establish a semi-classical trace formula for the induced quantization of symplectic maps, and give an application to Witten's asymptotic expansion conjecture for the quantum representations of the mapping class group.
\end{abstract}

\section{Introduction}

Geometric quantization is a set of geometric methods to construct a quantum mechanical system, represented by a Hilbert space of quantum states, from the underlying system of classical mechanics, represented by a symplectic manifold $(X, \omega)$. In this context, we require $(X, \omega)$ to be prequantized, so that $X$ is endowed with a Hermitian line bundle $\left(L, h^{L}\right)$ together with a Hermitian connection $\nabla^{L}$ of curvature $R^{L} \in \Omega^{2}(X, \mathbb{C})$ satisfying

$$
\omega=\frac{\sqrt{-1}}{2 \pi} R^{L} .
$$

Assume now that $(X, \omega)$ admits a compatible integrable complex structure $J \in \operatorname{End}(T X)$, making $(X, J, \omega)$ into a Kähler manifold and $\left(L, h^{L}, \nabla^{L}\right)$ into a holomorphic Hermitian line bundle equipped with its Chern connection. Then the Kähler quantization of $(X, \omega)$ at level $p \in \mathbb{N}^{*}$ is the Hilbert space $\mathscr{H}_{p}$ of $L^{2}$-holomorphic sections of $L^{p}:=L^{\otimes p}$ for the natural $L^{2}$-Hermitian product (2.8). The integer $p \in \mathbb{N}^{*}$ represents a quantum number, usually inversely proportional to the Planck constant, and asymptotic results when $p$ tends to infinity are then supposed to describe the so-called semi-classical limit, when the scale gets so large that we recover the laws of classical mechanics as an approximation of the laws of quantum mechanics. In this paper, we will restrict to the case of $(X, \omega)$ compact of dimension $\operatorname{dim} X=2 n$, so that $(X, \omega)$ represents a bounded mechanical system and the associated Hilbert space of quantum states $\mathscr{H}_{p}$ is finite dimensional for all $p \in \mathbb{N}^{*}$. 
A fundamental problem in this context is the dependence of the quantization on the choice of a complex structure $J \in \operatorname{End}(T X)$. A natural way to study this question is to consider the quantization of $(X, \omega)$ at level $p \in \mathbb{N}^{*}$ as a Hermitian vector bundle $\mathscr{H}_{p}$ over a space $B$ of compatible complex structures, whose fibre at $b \in B$ identifies with the induced Hilbert space $\mathscr{H}_{p, b}$ of holomorphic sections. One can then compare the quantizations associated with different complex structures via parallel transport with respect to the natural $L^{2}$-connection (2.18) on the vector bundle $\mathscr{H}_{p}$ over $B$. This point of view can be applied in particular to the quantization of symplectic maps, that is diffeomorphisms $\varphi: X \rightarrow X$ preserving $\omega$, which we require in addition to lift to the prequantization $\left(L, h^{L}, \nabla^{L}\right)$. In the particular case when $\varphi: X \rightarrow X$ preserves the complex structure, one can define its quantization at level $p \in \mathbb{N}^{*}$ as the induced unitary operator on holomorphic sections of $L^{p}$, but a symplectic map does not preserves any compatible complex structure in general. Instead, consider a path

$$
\left\{J_{t} \in \operatorname{End}(T X)\right\}_{t \in \mathbb{R}}
$$

of compatible complex structures such that $J_{0}:=J$ and $J_{1}:=\varphi^{*} J$. Then for any $p \in \mathbb{N}^{*}$, there is an induced pullback map $\varphi_{p}^{*}: \mathscr{H}_{p, 1} \rightarrow \mathscr{H}_{p, 0}$ from the space of holomorphic sections of $L^{p}$ with respect to $J_{1}$ to the space of holomorphic sections of $L^{p}$ with respect to $J_{0}$. One can then consider the parallel transport $\mathcal{T}_{p}: \mathscr{H}_{p, 0} \rightarrow \mathscr{H}_{p, 1}$ with respect to the $L^{2}$-connection along this path to get by composition a unitary operator

$$
\varphi_{p}^{*} \mathcal{T}_{p}: \mathscr{H}_{p, 0} \longrightarrow \mathscr{H}_{p, 0}
$$

giving a geometric definition of the quantization of the symplectic map $\varphi: X \rightarrow X$.

In this paper, we use the theory of Bergman kernels of Ma and Marinescu in [42] to study the parallel transport $\mathcal{T}_{p}$ as $p \rightarrow+\infty$. To describe our main result, let $\pi$ : $\mathbb{R} \times X \rightarrow \mathbb{R}$ be the fibration of complex manifolds with fibre $X_{t}:=\left(X, J_{t}\right)$ at $t \in$ $\mathbb{R}$, and write $\tau_{t}^{K_{X}}: K_{X_{0}} \rightarrow K_{X_{t}}$ for the parallel transport in the induced relative canonical line bundle $K_{X}:=\operatorname{det}\left(T^{(1,0) *} X\right)$ over $\mathbb{R} \times X$ with respect to the natural connection (5.4) along the horizontal directions of the fibration. Using the identification $T^{(0,1)} X_{t} \simeq T^{(1,0) *} X_{t}$ induced by the $\mathbb{C}$-bilinear form $g_{t}^{T X}:=\omega\left(\cdot, J_{t} \cdot\right)$ over $T X_{\mathbb{C}}$ for all $t \in \mathbb{R}$, we denote by $\operatorname{det}\left(\overline{\Pi_{t}^{0}}\right): K_{X, 0} \rightarrow K_{X, t}$ the line bundle isomorphism induced by the projection $\overline{\Pi_{t}^{0}}: T^{(0,1)} X_{0} \rightarrow T^{(0,1)} X_{t}$ on $T^{(0,1)} X_{t}$ with kernel $T^{(1,0)} X_{0}$ inside $T X_{\mathbb{C}}$. The following theorem, which follows from Theorem 3.16 and Lemma 5.1, expresses the parallel transport $\mathcal{T}_{p, t}: \mathscr{H}_{p, 0} \rightarrow \mathscr{H}_{p, t}$ with respect to the $L^{2}$-connection (2.18) as a Toeplitz operator from one quantum space to another, and is the central result of this paper.

Theorem 1.1. There exists a family of functions $\left\{\mu_{l, t} \in \mathscr{C}^{\infty}(X, \mathbb{C})\right\}_{l \in \mathbb{N}}$, smooth in $t \in[0,1]$, such that for all $k \geqslant 0$, there exists $C_{k}>0$ such that for all $p \in \mathbb{N}^{*}$,

$$
\left\|\mathcal{T}_{p, t}-\sum_{l=0}^{k-1} p^{-l} P_{p, t} \mu_{l, t} P_{p, 0}\right\| \leqslant C_{k} p^{-k},
$$


in operator norm, where $P_{p, t}: \mathscr{C}^{\infty}\left(X, L^{p}\right) \rightarrow \mathscr{H}_{p, t}$ is the orthogonal projection with respect to the $L^{2}$-Hermitian product on the space of holomorphic sections of $L^{p}$ over $X_{t}$.

Furthermore, via the canonical isomorphism $\operatorname{End}\left(K_{X, 0}\right) \simeq \mathbb{C}$, we have the following formula for the first coefficient,

$$
\bar{\mu}_{0, t}^{2}=\operatorname{det}\left(\overline{\Pi_{t}^{0}}\right)^{-1} \tau_{t}^{K_{X}} .
$$

Theorem 1.1 generalizes and refines the asymptotic expansion established by Andersen in [1, Th.6] for the parallel transport in the endomorphism bundle End $\left(\mathscr{H}_{p}\right)$ as $p \rightarrow+\infty$ induced by Hitchin connections, as defined in Section 5.2 following [2, $\S 1]$. In particular, Andersen uses his expansion to establish in [1, Th.1] the asymptotic faithfulness of the quantum representations of the mapping class group, while our explicit formula (1.5) for the first term of the expansion (1.4) will be crucial here to obtain Witten's formula for the quantum representations of the mapping class group in Theorems 1.3 and 1.4.

On the other hand, Theorem 1.1 has already been applied in [34] to the quantization of Hamiltonian flows $\varphi_{t}: X \rightarrow X, t \in \mathbb{R}$, via the parallel transport over the induced path $\left\{\varphi_{t}^{*} J \in \operatorname{End}(T X)\right\}_{t \in \mathbb{R}}$ of complex structures. In particular, our explicit formula (1.5) for the first coefficient is used in [34, Th.1.2, Th.1.3] to establish a Gutzwiller trace formula in this context. The parallel transport over complex structures induced by Hamiltonian flows has also been studied by Foth and Uribe in [30], where it is shown that it satisfies a Schrödinger equation, and by Charles in [26], who studies its semi-classical properties in the metaplectic case.

In Section 4, we use Theorem 1.1 to estimate the trace of the quantization (1.3) of a symplectic map $\varphi: X \rightarrow X$ as $p \rightarrow+\infty$, showing in particular that it localizes around the fixed point set $X^{\varphi} \subset X$ of $\varphi$. In the following theorem, which follows from Theorem 4.3 and Lemma 5.2, we assume for simplicity that $X^{\varphi}$ is connected.

Theorem 1.2. Assume that $X^{\varphi}$ is a smooth submanifold with $T X^{\varphi}=\operatorname{Ker}\left(\operatorname{Id}_{T X}-d \varphi\right)$. Then there are densities $\nu_{r}$ over $X^{\varphi}$ for all $r \in \mathbb{N}$, such that for any $k \in \mathbb{N}$ and as $p \rightarrow+\infty$,

$$
\operatorname{Tr}_{\mathscr{H}_{p}}\left[\varphi_{p}^{*} \mathcal{T}_{p}\right]=p^{\frac{\operatorname{dim} X^{\varphi}}{2}} \lambda^{p}\left(\sum_{r=0}^{k-1} p^{-r} \int_{X^{\varphi}} \nu_{r}+O\left(p^{-k}\right)\right),
$$

where $\bar{\lambda} \in \mathbb{C}$ is the value of the action of $\varphi: X \rightarrow X$ on $L$ over $X^{\varphi}$. Furthermore, there is an explicit local formula for $\nu_{0}$, given in (4.27).

If $X^{\varphi}$ is a complex submanifold of $X$ and if $\varphi$ preserves a complex subbundle $N$ transverse to $T X^{\varphi} \subset T X$ over $X^{\varphi}$, then

$$
\nu_{0}=(-1)^{\frac{2 n-\operatorname{dim} X^{\varphi}}{4}}\left(\varphi^{K_{X}} \tau^{K_{X},-1}\right)^{1 / 2} \operatorname{det}_{N}\left(\operatorname{Id}_{N}-\left.d \varphi\right|_{N}\right)^{-1 / 2}|d v|_{T X / N},
$$

for some natural choices of square roots, where $\tau^{K_{X}}: K_{X_{0}} \rightarrow K_{X_{1}}$ is the parallel transport with respect to the natural connection (5.4) and $|d v|_{T X / N}$ is the density over $X^{\varphi}$ induced by $g_{0}^{T X}:=\omega\left(\cdot, J_{0} \cdot\right)$ and the decomposition $T X=T X^{\varphi} \oplus N$ as in (4.25). 
Note that the assumption of (1.7) is automatically satisfied when $\operatorname{dim} X^{\varphi}=0$ or when $\varphi$ is holomorphic. The general version of Theorem 1.2, which is Theorem 4.3, gives a general formula for the higher order term of (1.6) without the assumption of (1.7), and includes the tensor product with a general Hermitian vector bundle $E$ over the fibration $\pi: \mathbb{R} \times X \rightarrow \mathbb{R}$. In Section 4.1, we first establish the general version of Theorem 1.2 as above in the case $\operatorname{dim} X^{\varphi}=0$, which generalizes a result of Charles in [27, Th.5.3.1], who only handles the metaplectic case, that is taking $E=K_{X}^{1 / 2}$ to be a square root of the relative canonical bundle. He then applies it in [28, Th.1.2] to prove an asymptotic projective version of Witten's asymptotic conjecture for the quantum representations of the mapping class group. As we will see below, it is crucial to be able to consider the case without metaplectic correction to handle the actual conjecture, as formulated in the language of modular functors of Segal in $[48, \S 5]$. Furthermore, Theorem 1.2 deals with $X^{\varphi}$ of arbitrary dimension, which allows to extend the conjecture to that case.

In case the symplectic map $\varphi$ preserves the complex structure, so that the pullback map $\varphi_{p}^{*}$ preserves $\mathscr{H}_{p}$ for all $p \in \mathbb{N}^{*}$, we recover via Theorem 4.3 the asymptotics of $\operatorname{Tr}_{\mathscr{H}_{p}}\left[\varphi_{p}^{*}\right]$ as $p \rightarrow+\infty$ of the following equivariant Riemann-Roch-Hirzebruch formula

$$
\operatorname{Tr}_{\mathscr{H}_{p}}\left[\varphi_{p}^{*}\right]=\int_{X^{\varphi}} \operatorname{Td}_{\varphi^{-1}}\left(T^{(1,0)} X\right) \operatorname{ch}_{\varphi^{-1}}\left(L^{p}\right)
$$

where $\operatorname{Td}_{\varphi^{-1}}\left(T^{(1,0)} X\right)$ represents the equivariant Todd class of $(X, J)$ and $\operatorname{ch}_{\varphi^{-1}}\left(L^{p}\right)$ represents the equivariant Chern character of $L^{p}$ for the action induced by $\varphi^{-1}$, as defined in [19, Def. 1.5].

In Section 5, we show how Theorem 1.2 can be applied to establish Witten's asymptotic expansion conjecture for the quantum representations of the mapping class group, as described for instance in [3, Conj.1.1], and to compute the formula given by Witten in $[50,(2.17)]$ for the first coefficient. For simplicity and concreteness, we will only consider the case $X:=\mathscr{M}$ to be the moduli space of gauge equivalence classes of flat $\mathrm{SU}(m)$-connections over $\Sigma \backslash D$ with holonomy around the boundary equal to $e^{\frac{2 \sqrt{-1} \pi d}{m}}$ for some fixed $d \in(\mathbb{Z} / m \mathbb{Z})^{*}$, where $\Sigma$ is a compact oriented surface of genus $g \geqslant 2$ and $D \subset \Sigma$ is an embedded disk. Then as explained in Propositions 5.9 and 5.10, the moduli space $\mathscr{M}$ admits a natural structure of a compact prequantized symplectic manifold. We then consider the map $\varphi: \mathscr{M} \rightarrow \mathscr{M}$ naturally induced by an orientation preserving diffeomorphism $f \in \operatorname{Diff}^{+}(\Sigma, D)$ preserving $D$ pointwise. Then $\varphi$ is a smooth symplectic map lifting naturally to the prequantization, and only depends on $f \in \operatorname{Diff}^{+}(\Sigma, D)$ up to isotopies of $\Sigma$ preserving $D$, so that it represents an element of the mapping class group of $\Sigma$ acting symplectically on $\mathscr{M}$.

An element $\sigma \in \mathscr{T}_{\Sigma}$ of the Teichmüller space of $\Sigma$ induces canonically a compatible complex structure $J_{\sigma} \in \operatorname{End}(T \mathscr{M})$, and we can consider the associated quantum bundle $\mathscr{H}_{p}$ over $\mathscr{T}_{\Sigma}$ for all $p \in \mathbb{N}^{*}$, called the Verlinde bundle. It is endowed with a canonical connection for all $p \in \mathbb{N}^{*}$, which was introduced independently by Hitchin in [33, Th.3.6] and Axelrod, S. Della Pietra and Witten in [12, §4.b]. Note that this canonical connection is defined in [33] only up to an additive scalar form, which is fixed canonically in [12]. Let $\mathcal{T}_{p}$ be the induced parallel transport along a path $\gamma:[0,1] \rightarrow \mathscr{T}_{\Sigma}$ joining 
$\sigma \in \mathscr{T}_{\Sigma}$ to $f^{*} \sigma \in \mathscr{T}_{\Sigma}$. Then the trace $\operatorname{Tr}_{\mathscr{H}_{p}}\left[\varphi^{*} \mathcal{T}_{p}\right]$ is the anomalous Witten-ReshetikhinTuraev invariant of the mapping torus $\Sigma_{f}$ with one link lifting the canonical fibration $\pi_{f}: \Sigma_{f} \rightarrow \mathbb{R} / \mathbb{Z}$ colored by $e^{\frac{2 \sqrt{-1} \pi d}{m}}$, as considered in Segal's definition of conformal field theory in $[48, \S 4]$ after Witten's description in $[50,(2.1)]$ using path integrals.

In fact, let $\mathscr{M}_{f}$ be the moduli space of gauge equivalence classes of flat $\mathrm{SU}(m)$ connections over the mapping torus $(\Sigma \backslash D)_{f}$ of $f \in \operatorname{Diff}^{+}(\Sigma, D)$ with holonomy around the boundary equal to $e^{\frac{2 \sqrt{-1} \pi d}{m}}$, and assume that $\mathscr{M}_{f}$ admits a smooth structure as in Proposition 5.9. Then there is a smooth covering $r: \mathscr{M}_{f} \rightarrow \mathscr{M}^{\varphi}$ defined by restriction on any fibre, where $\mathscr{M}^{\varphi} \subset \mathscr{M}$ is the fixed point set of $\varphi$. For any $\left[A_{f}\right] \in \mathscr{M}_{f}$, the Chern-Simons invariant of $\left[A_{f}\right] \in \mathscr{M}_{f}$ is given by the formula

$$
\mathrm{CS}\left(\left[A_{f}\right]\right)=\frac{m}{8 \pi^{2}}\left(\operatorname{Tr}\left[\xi_{1} \xi_{2}\right]+\int_{\Sigma_{f}} \operatorname{Tr}\left[\alpha_{f} \wedge d \alpha_{f}+\frac{2}{3} \alpha_{f} \wedge \alpha_{f} \wedge \alpha_{f}\right]\right) \in \mathbb{R} / \mathbb{Z},
$$

where we took a connection form $\alpha_{f} \in \Omega^{1}\left(\Sigma_{f}, \mathfrak{s u}(m)\right)$ of $\left[A_{f}\right]$ equal to $\xi_{1} d \theta_{1}+\xi_{2} d \theta_{2}$ on the boundary of $(\Sigma / D)_{f}$ seen as a torus with coordinates $\theta_{1}, \theta_{2} \in S^{1}$, with $\xi_{1}, \xi_{1} \in \mathfrak{s u}(m)$ constant.

On the other hand, the path $\gamma:[0,1] \rightarrow \mathscr{T}_{\Sigma}$ in Teichmüller space determines a complex structure on the relative tangent bundle of the canonical fibration $\pi_{f}: \Sigma_{f} \rightarrow$ $\mathbb{R} / \mathbb{Z}$ up to isotopy, and we endow the fibres with the compatible hyperbolic Riemannian metric. For any $\left[A_{f}\right] \in \mathscr{M}_{f}$, write $\left[\operatorname{ad} A_{f}\right]$ for the induced connection on the trivial adjoint $\mathfrak{s u}(m)$-bundle over the whole $\Sigma_{f}$.

Theorem 1.3. Let $\mathscr{M}_{f}=\coprod_{j=1}^{q} \mathscr{M}_{f, j}$ be the decomposition into connected components. Then there exist densities $\nu_{r}$ over $\mathscr{M}_{f}$ for all $r \in \mathbb{N}$, such that for any $k \in \mathbb{N}$, the anomalous Witten-Reshetikhin-Turaev invariant satisfies as $p \rightarrow+\infty$,

$$
\operatorname{Tr}_{\mathscr{H}_{p}}\left[\varphi_{p}^{*} \mathcal{T}_{p}\right]=\frac{1}{m} \sum_{j=1}^{q} p^{\frac{\operatorname{dim} \mathscr{M}_{f, j}}{2}} e^{2 \sqrt{-1} \pi p \mathrm{CS}_{j}}(\sqrt{-1})^{k_{j}}\left(\sum_{r=0}^{k-1} p^{-r} \int_{\mathscr{M}_{f, j}} \nu_{r}+O\left(p^{-k}\right)\right)
$$

where $k_{j} \in \mathbb{Z} / 4 \mathbb{Z}$ and where $\mathrm{CS}_{j} \in \mathbb{R} / \mathbb{Z}$ is the constant value of the Chern-Simons invariant (1.9) over $\mathscr{M}_{f, j}$. Furthermore, there is an explicit local formula for $\nu_{0}$.

If $r: \mathscr{M}_{f} \rightarrow \mathscr{M}_{\Sigma}$ is holomorphic and if $\varphi$ preserves a complex subbundle transverse to $T \mathscr{M}^{\varphi} \subset T \mathscr{M}$ over $\mathscr{M}^{\varphi}$, we get

$$
\nu_{0}\left(\left[A_{f}\right]\right)=\exp \left(\frac{\sqrt{-1} \pi}{4} \eta^{0}\left(\operatorname{ad} A_{f}\right)\right)\left|\tau_{\Sigma_{f}}\left(\operatorname{ad} A_{f}\right)\right|^{1 / 2},
$$

for any $\left[A_{f}\right] \in \mathscr{M}_{f}$, where $\eta^{0}\left(\operatorname{ad} A_{f}\right)$ is the adiabatic limit of the $\eta$-invariant of the odd signature operator of ad $A_{f}$ restricted to odd forms over the Riemannian fibration $\pi_{f}: \Sigma_{f} \rightarrow \mathbb{R} / \mathbb{Z}$, and $\left|\tau_{\Sigma_{f}}\left(\operatorname{ad} A_{f}\right)\right|^{1 / 2}$ is the square root of the absolute value of the Reidemeister torsion of ad $A_{f}$, seen as a density over $\mathscr{M}_{f}$ via Poincaré duality.

Note that the hypothesis of (1.11) is automatically satisfied when $\operatorname{dim} \mathscr{M}_{f}=0$ or when $f$ preserves $\sigma \in \mathscr{T}_{\Sigma}$. The presence of the $\eta$-invariant in the formula (1.11) is 
a consequence of the holonomy theorem of Bismut and Freed in [15, Th.3.16] as well as the study of holomorphic determinant line bundles of Bismut, Gillet and Soule in $[18, \S 1]$, and depends on the chosen path in Teichmüller space. On the other hand, as showed in [33, Th.4.9] and in [12, §4.b], the canonical connection over the Verlinde bundle is projectively flat. By the explicit computation of its curvature in $[12, \S 4 . \mathrm{b}]$ and in the language of modular functors of [48, (5.9)], the Witten-Reshetikhin-Turaev invariant of the mapping torus with one colored link as above is computed by the trace $\operatorname{Tr} \widetilde{\mathscr{H}}_{p}\left[\varphi_{p}^{*} \mathcal{T}_{p}\right]$, where $\mathcal{T}_{p}$ is the flat parallel transport in

$$
\widetilde{\mathscr{H}_{p}}:=\mathscr{H}_{p} \otimes \operatorname{det}\left(\bar{\partial}_{\Sigma}\right)^{-\frac{\left(m^{2}-1\right) p}{2(p+m)}}
$$

induced by the canonical projectively flat connection on $\mathscr{H}_{p}$ and the Chern connection associated with the Quillen metric on the holomorphic determinant line bundle $\operatorname{det}\left(\bar{\partial}_{\Sigma}\right)$ of the universal family of $\bar{\partial}$-operators over $\mathscr{T}_{\Sigma}$, for which fractional powers exist as Teichmüller space is contractible and on which $\varphi$ lifts naturally up to choices of compatible lifts on each fractional power. The following result then computes its first order as $p \rightarrow+\infty$.

Theorem 1.4. The Witten-Reshetikhin-Turaev invariant defined above satisfies the expansion (1.10), with first coefficient as in (1.11) given for any $\left[A_{f}\right] \in \mathscr{M}_{f}$ by

$$
\nu_{0}\left(\left[A_{f}\right]\right)=\exp \left(\frac{\sqrt{-1} \pi}{4} \rho\left(\operatorname{ad} A_{f}\right)\right)\left|\tau_{\Sigma_{f}}\left(\operatorname{ad} A_{f}\right)\right|^{1 / 2},
$$

where $\rho\left(\operatorname{ad} A_{f}\right)$ is the topological $\rho$-invariant of ad $A_{f}$ defined in (5.35).

As explained for instance in [3, p.10], the choice of a lift of $\varphi$ to fractional powers of $\operatorname{det}\left(\bar{\partial}_{\Sigma}\right)$ corresponds to the canonical central extension of the mapping class group induced by the Atiyah 2-framing of [9], which can be used to get the corresponding correction in $[50,(2.23)]$. The map associating an element of this extension to the endomorphism $\varphi_{p}^{*} \mathcal{T}_{p} \in \operatorname{End}\left(\widetilde{\mathscr{H}}_{p}\right)$ is called a quantum representation of the mapping class group. Another approach followed by Charles in $[28, \S 7]$ is to consider the metaplectic correction, replacing $L^{p}$ by $L^{p} \otimes K_{\mathscr{M}}^{1 / 2}$ for all $p \in \mathbb{N}^{*}$. However, the corresponding trace does not coincide with the Witten-Reshetikhin-Turaev invariant, and this approach does not imply Theorem 1.3 nor Theorem 1.4, even in the case $\operatorname{dim} \mathscr{M}_{f}=0$, which is the only one handled in [28, Th.1.2]. By Lemma 5.8, we recover [28, Th.1.2] as a consequence of the general version of Theorem 1.2.

The Verlinde bundle with its canonical connection is defined for much more general moduli spaces, the one considered above being the standard smooth model. The above approach applies as soon as the moduli space has a natural smooth structure, and Theorems 1.1 and 1.2 should extend to the case of orbifolds, so that one could get the expansion (1.10) for much more general quantum representations of the mapping class group. In the case when $f \in \operatorname{Diff}^{+}(\Sigma, D)$ preserves $\sigma \in \mathscr{T}_{\Sigma}$, so that $\varphi: \mathscr{M} \rightarrow$ $\mathscr{M}$ preserves $J_{\sigma} \in \operatorname{End}(T \mathscr{M})$, Andersen applies in [3, Th.1.1] a singular version of 
the equivariant Riemann-Roch-Hirzebruch formula (2.13) to establish the asymptotic expansion for general singular moduli spaces, and Andersen and Himpel identify the first coefficient with (1.13) in [5, Th.1.6]. In [6, Th.1.2], Andersen and Petersen work in the case of Hitchin connections of Section 5.2, but under a weaker assumption on the fixed point set, to get the asymptotic expansion (1.10) for a larger class of mapping tori. However, they do not compute the first coefficient.

This paper is based on the theory of Berezin-Toeplitz operators of Ma and Marinescu in [43], and can be seen as an extension of this theory to families. More specifically, we work in the context of the Berezin-Toeplitz quantization introduced in [35] of general prequantized symplectic manifolds, using the spaces $\mathscr{H}_{p}$ of almost holomorphic sections of $L^{p}$ for all $p \in \mathbb{N}^{*}$ large enough, defined as the direct sum of the eigenspaces associated with the small eigenvalues of the renormalized Bochner Laplacian (2.10) of Guillemin and Uribe [32]. These spaces coincide with the space of holomorphic sections for $p \in \mathbb{N}^{*}$ large enough in the Kähler case, and Theorems 3.16 and 4.3, which are the general versions of Theorems 1.1 and 1.2, are proved in this generality. A family version of Berezin-Toeplitz quantization has first been studied by Ma and Zhang in [44], where they show that the curvature of the quantum bundle with respect to the $L^{2}$-connection is a Toeplitz operator, and compute the first two coefficients.

The theory of Berezin-Toeplitz operators in the Kähler case was first developed by Bordemann, Meinreken and Schlichenmaier in [23] and Schlichenmaier in [47]. Their approach is based on the work of Boutet de Monvel and Sjöstrand on the Szegö kernel in [25], and the theory of Toeplitz structures developed by Boutet de Monvel and Guillemin in [24]. In this context, Zelditch first introduced in [51] a quantization of symplectic maps based on a unitary version of [24], which does not depend on the choice of a path of complex structures. This quantization is defined up to a phase function in its symbol, while the amplitude [51, (3.10)] of its symbol coincides with the amplitude (3.21) of the local model in flat space on the diagonal, which does not depend on a path of complex structures. This shows via Lemma 5.3 that the first coefficient (1.5) in Theorem 1.1 also depends on the path only up to a phase function. The local model of Section 3.1 has also been considered in another form by Kirwin and $\mathrm{Wu}$ in [37], where they deal with paths of complex structures on flat space given by geodesics in the Siegel upper half-space. In particular, we recover [37, Th.3.4] as a special case of Proposition 3.2.

In Section 2, we introduce the basic notions of fibrations used in this paper, and recall the results from the theory of the Bergman kernel of [41] in this setting. In Section 3, we study the composition of Bergman kernels associated with different complex structures, introduce the corresponding generalization of a Toeplitz operator and establish the general version of Theorem 1.1. In Section 4, we use this result to establish the general version of Theorem 1.2. In Section 5, we introduce the notion of a Hitchin connection and relate it to our notion of Toeplitz connection, then apply our results to establish Theorems 1.3 and 1.4 .

Acknowledgements. The author wants to thank Prof. Xiaonan Ma for his constant support, and Long Mai for a useful discussion on the last section of this paper. The 
author also wants to thank the anonymous referee for useful comments and suggestions. This work was supported by the grant DIM-RDF from Région Ile-de-France.

\section{Prequantized fibrations and Bergman kernels}

In Section 2.1, we introduce the notion of a prequantized fibration and construct the associated quantum bundle over its base. In Section 2.2, we recall the results of [41] in this setting. In Section 2.3, we specialize these notions in the case of a trivialized fibration.

\subsection{Setting}

Let $\pi: M \rightarrow B$ be a submersion of smooth manifolds with compact fibre $X$ such that $\operatorname{dim} X=2 n$, and assume $B$ connected. Let $T X$ be the associated relative tangent bundle over $M$, defined as the kernel of $d \pi$ inside the tangent bundle $T M$ of $M$.

Definition 2.1. A submersion $\pi: M \rightarrow B$ as above together with a Hermitian line bundle with Hermitian connection $\left(L, h^{L}, \nabla^{L}\right)$ over $M$ is called a prequantized fibration if the restriction of the curvature $R^{L} \in \Omega^{2}(M, \mathbb{C})$ of $\nabla^{L}$ to $T X \subset T M$ is non degenerate.

The relative symplectic form $\omega \in \Omega^{2}(M, \mathbb{R})$ of the fibration is defined by (1.1), and induces by restriction a symplectic structure on the fibres of $\pi$. For any $x \in M$, let $T_{x}^{H} M$ be the subspace of $T_{x} M$ defined by

$$
T_{x}^{H} M=\left\{v \in T_{x} M \mid \omega(v, u)=0, \forall u \in T_{x} X\right\} .
$$

Then (2.1) defines a subbundle $T^{H} M$ of $T M$, called the horizontal tangent bundle. We have

$$
T M=T X \oplus T^{H} M
$$

and $d \pi$ induces an isomorphism between $T^{H} M$ and $\pi^{*} T B$ over $M$. For any $x \in M$ and $v \in T_{\pi(x)} B$, we write $v_{x}^{H} \in T_{x}^{H} M$ for its horizontal lift via (2.2), so that $d \pi \cdot v_{x}^{H}=v$. Let $P^{X}, P^{H}$ be the projections from $T M$ to $T X, T^{H} M$, and for any form $\alpha \in \Omega^{\bullet}(M, \mathbb{C})$, write $\alpha^{X}, \alpha^{H}$ for the restriction of $\alpha$ to $T X, T^{H} M$. Then (2.1) implies $\omega=\omega^{X}+\omega^{H}$ via $(2.2)$.

Let $J \in \operatorname{End}(T X)$ over $M$ satisfying $J^{2}=-\operatorname{Id}_{T X}$ be compatible with $\omega$, so that $\omega$ is $J$-invariant and that $g^{T X}:=\omega(\cdot, J \cdot)$ defines an Euclidean metric on $T X$ over $M$. We call $J \in \operatorname{End}(T X)$ a relative almost complex structure over $M$ compatible with $\omega$, and $g^{T X}$ the associated relative Riemannian metric.

Let $g^{T B}$ be a metric on $T B$, which we lift to a metric on $T^{H} M$ via $d \pi$. Using (2.2), we endow $T M$ with the unique metric $g^{T X} \oplus g^{T B}$ restricting to $g^{T X}, g^{T B}$ on $T X, T B$ and for which $T X$ and $T^{H} M$ are orthogonal. Write $\nabla^{T M}$ for the associated Levi-Civita connection on $T M$, and following Bismut in [14, Def. 1.6], define the vertical Levi-Civita connection $\nabla^{T X}$ on $T X$ by the formula

$$
\nabla^{T X}=P^{X} \nabla^{T M} P^{X}
$$


Then $\nabla^{T X}$ induces the Levi-Civita connection of $g^{T X}$ on the fibres of $\pi$, and by [17, Th.1.2], this definition does not depend on the choice of $g^{T B}$.

If $h^{F}$ is a Hermitian metric on a complex vector bundle $F$, we write $\langle\cdot, \cdot\rangle_{F}$ for the associated Hermitian product. Let $T X_{\mathbb{C}}=T X \otimes_{\mathbb{R}} \mathbb{C}$ be the complexification of $T X$. The complex structure $J$ on $T X$ induces a splitting

$$
T X_{\mathbb{C}}=T^{(1,0)} X \oplus T^{(0,1)} X
$$

into the eigenspaces of $J$ corresponding to the eigenvalues $\sqrt{-1}$ and $-\sqrt{-1}$ respectively. We denote by $P^{(1,0)}, P^{(0,1)}$ the corresponding projections from $T X_{\mathbb{C}}$ to $T^{(1,0)} X, T^{(0,1)} X$. Let $h^{T^{(1,0)} X}, h^{T^{(0,1)}} X$ be the Hermitian metrics on $T^{(1,0)} X, T^{(0,1)} X$ induced by $g^{T X}$ via (2.4), and define Hermitian connections on $\left(T^{(1,0)} X, h^{T^{(1,0)} X}\right),\left(T^{(0,1)} X, h^{T^{(0,1)} X}\right)$ by

$$
\begin{aligned}
& \nabla^{T^{(1,0)} X}=P^{(1,0)} \nabla^{T X} P^{(1,0)} \\
& \nabla^{T^{(0,1)} X}=P^{(0,1)} \nabla^{T X} P^{(0,1)} .
\end{aligned}
$$

The relative canonical line bundle is the line bundle $K_{X}=\operatorname{det}\left(T^{(1,0) *} X\right)$ over $M$, equipped with $h^{K_{X}}, \nabla^{K_{X}}$ induced by $h^{T^{(0,1)} X}, \nabla^{T^{(0,1)} X}$. Let $\left(E, h^{E}, \nabla^{E}\right)$ be a Hermitian vector bundle with Hermitian connection over $M$. For any $p \in \mathbb{N}^{*}$, write $L^{p}$ for the $p$-th tensor power of $L$, and set

$$
E_{p}=L^{p} \otimes E
$$

Let $h^{E_{p}}, \nabla^{E_{p}}$ be induced by $h^{L}, h^{E}$ and $\nabla^{L}, \nabla^{E}$ on $E_{p}$.

For any $b \in B$, set $X_{b}=\pi^{-1}(b)$. Let $\omega_{b}$ and $g_{b}^{T X}$ be the symplectic form and Riemannian metric induced on $X_{b}$ by restriction of $\omega$ and $g^{T X}$, and let $d v_{X_{b}}$ be the volume form on $X_{b}$ defined by

$$
d v_{X_{b}}=\frac{\omega_{b}^{n}}{n !}
$$

Then $d v_{X_{b}}$ is the Riemannian volume form of $\left(X_{b}, g_{b}^{T X}\right)$ compatible with the orientation induced by $\omega_{b}$. Let $L_{b}, E_{b}, E_{p, b}, T^{(1,0)} X_{b}$ be the vector bundles over $X_{b}$ induced by restriction of $L, E, E_{p}, T^{(1,0)} X$. We can then see $\mathscr{C}^{\infty}\left(M, E_{p}\right)$ as the space of smooth sections of an infinite dimensional vector bundle with fibre $\mathscr{C}^{\infty}\left(X_{b}, E_{p, b}\right)$ at $b \in B$. For any $p \in \mathbb{N}^{*}$, we endow it with the $L^{2}$-Hermitian product $\langle\cdot, \cdot\rangle_{p}$, defined for any $b \in B$ and $s_{1}, s_{2} \in \mathscr{C}^{\infty}\left(X_{b}, E_{p, b}\right)$ by

$$
\left\langle s_{1}, s_{2}\right\rangle_{p, b}=\int_{X_{b}}\left\langle s_{1}(x), s_{2}(x)\right\rangle_{E_{p, b}} d v_{X_{b}}(x)
$$

For any $p \in \mathbb{N}^{*}$, the Bochner Laplacian $\Delta^{E_{p}}$ is the differential operator acting on $\mathscr{C}^{\infty}\left(M, E_{p}\right)$ by the formula

$$
\Delta^{E_{p}}=-\sum_{j=1}^{2 n}\left[\left(\nabla_{e_{j}}^{E_{p}}\right)^{2}-\nabla_{\nabla_{e_{j}}^{T X} e_{j}}^{E_{p}}\right]
$$


where $\left\{e_{j}\right\}_{j=1}^{2 n}$ is any local orthonormal frame of $\left(T X, g^{T X}\right)$. For any $p \in \mathbb{N}^{*}$ and any Hermitian endomorphism $\Psi \in \mathscr{C}^{\infty}(M, \operatorname{End}(E))$, the renormalized Bochner Laplacian is the second order differential operator $\Delta_{p, \Psi}$ acting on $\mathscr{C}^{\infty}\left(M, E_{p}\right)$ by

$$
\Delta_{p, \Psi}=\Delta^{E_{p}}-2 \pi n p-\Psi
$$

where $\Psi$ denotes the operator of pointwise multiplication by $\Psi$. We fix the endomorphism $\Psi \in \mathscr{C}^{\infty}(M, \operatorname{End}(E))$ and simply write $\Delta_{p}$ for the associated renormalized Bochner Laplacian.

For any $b \in B$ and $p \in \mathbb{N}^{*}$, let $L^{2}\left(X_{b}, E_{p, b}\right)$ be the completion of $\mathscr{C}^{\infty}\left(X_{b}, E_{p, b}\right)$ with respect to $\langle\cdot, \cdot\rangle_{p, b}$. Then $\Delta_{p}$ induces by restriction an elliptic self-adjoint operator $\Delta_{p, b}$ on $L^{2}\left(X_{b}, E_{p, b}\right)$, which by standard elliptic theory has discrete spectrum contained in $\mathbb{R}$. The following theorem comes essentially from [40, Cor.1.2], and can be deduced from the manifest uniformity in the parameters of its proof in $[40, \S 3]$.

Theorem 2.2. For any $U \subset \subset B$, there exist $\widetilde{C}, C>0$ such that for any $b \in U$ and $p \in \mathbb{N}^{*}$,

$$
\operatorname{Spec}\left(\Delta_{p, b}\right) \subset[-\widetilde{C}, \widetilde{C}] \cup[4 \pi p-C,+\infty[.
$$

Furthermore, the direct sum

$$
\mathscr{H}_{p, b}=\bigoplus_{\substack{\lambda \in \operatorname{Spec}\left(\Delta_{p, b}\right) \\ \lambda \in[-\widetilde{C}, \widetilde{C}]}} \operatorname{Ker}\left(\lambda-\Delta_{p, b}\right)
$$

is naturally included in $\mathscr{C}^{\infty}\left(X_{b}, E_{p, b}\right)$, and there is $p_{0} \in \mathbb{N}$ such that for any $b \in U$ and $p \geqslant p_{0}$, we have

$$
\operatorname{dim} \mathscr{H}_{p, b}=\int_{X} \operatorname{Td}\left(T^{(1,0)} X_{b}\right) \operatorname{ch}\left(E_{b}\right) \exp \left(p \omega_{b}\right)
$$

where $\operatorname{Td}\left(T^{(1,0)} X_{b}\right)$ represents the Todd class of $T^{(1,0)} X_{b}$ and $\operatorname{ch}\left(E_{b}\right)$ represents the Chern character of $E_{b}$.

The right hand side of (2.13) is an integer depending smoothly on $b \in B$, thus locally constant in $b \in B$. In view of Theorem 2.2, we will assume $B$ compact from now on, and fix $p_{0} \in \mathbb{N}$ with $\widetilde{C}<4 \pi p_{0}-C$ and such that (2.13) is satisfied for all $p \geqslant p_{0}$ and $b \in B$. As $B$ is connected, this implies that $\operatorname{dim} \mathscr{H}_{p, b}$ does not depend on $b \in B$. Following $[13, \S 9.2]$, we can define the orthogonal projection operator $P_{p, b}$ from $\mathscr{C}^{\infty}\left(X_{b}, E_{p, b}\right)$ to $\mathscr{H}_{p, b}$ with respect to the $L^{2}$-Hermitian product $(2.8)$ by the following contour integral in the complex plane,

$$
P_{p, b}=\int_{\Gamma}\left(\lambda-\Delta_{p, b}\right)^{-1} d \lambda
$$

where $\Gamma$ is a circle of center 0 and radius $a>0$ such that $\widetilde{C}<a<4 \pi p-C$. This shows that the projection operators $P_{p, b}$ depend smoothly on $b \in B$, and as the dimension of $\operatorname{Im}\left(P_{p, b}\right)=\mathscr{H}_{p, b}$ is constant in $b \in B$, this defines a finite dimensional bundle over $B$ 
with fibre $\mathscr{H}_{p, b}$ at $b \in B$. We write this bundle $\mathscr{H}_{p}$, and we endow it with the Hermitian structure $h^{\mathscr{H}_{p}}$ given by the $L^{2}$-Hermitian product (2.8) restricted to $\mathscr{H}_{p, b}$ for all $b \in B$. By convention, we take $\mathscr{H}_{p, b}=\{0\}$ for all $b \in B$, so that $\mathscr{H}_{p}$ is the trivial bundle over $B$, whenever $p<p_{0}$. The family $\left\{\mathscr{H}_{p}\right\}_{p \in \mathbb{N}^{*}}$ of Hermitian vector bundles over $B$ is called the quantum bundle of $\pi: M \rightarrow B$.

As $P_{p, b}$ is a projection operator on a finite dimensional space for all $p \in \mathbb{N}^{*}$, it has smooth Schwartz kernel with respect to $d v_{X_{b}}$, depending smoothly on $b \in B$. Specifically, let $M \times_{B} M$ be the fibred product of $M$ with itself over $B$, and write $\pi_{1}, \pi_{2}$ for the first and second projections from $M \times_{B} M$ to $M$. For any vector bundles $F_{1}$ and $F_{2}$ over $M$, we define the fibred tensor product of $F_{1}$ and $F_{2}$ over $B$ as a vector bundle $F_{1} \otimes_{B} F_{2}$ over $M \times_{B} M$ by the formula

$$
F_{1} \otimes_{B} F_{2}=\pi_{1}^{*} F_{1} \otimes \pi_{2}^{*} F_{2} .
$$

Then there exists $P_{p}(.,.) \in \mathscr{C}^{\infty}\left(M \times_{B} M, E_{p} \bigotimes_{B} E_{p}^{*}\right)$ such that for all $b \in B, s \in$ $\mathscr{C}^{\infty}\left(X_{b}, E_{p, b}\right)$ and $x \in X_{b}$, we get

$$
\left(P_{p, b} s\right)(x)=\int_{X_{b}} P_{p}(x, y) s(y) d v_{X_{b}}(y) .
$$

We call $P_{p}(\cdot, \cdot)$ the relative Bergman kernel of $E_{p}$ over $\pi: M \rightarrow B$. Taking $s \in$ $\mathscr{C}^{\infty}\left(M, E_{p}\right)$ in $(2.16)$, this defines in turn a projection operator $P_{p}$ from $\mathscr{C}^{\infty}\left(M, E_{p}\right)$ to $\operatorname{Ker}\left(\Delta_{p}\right)=\mathscr{C}^{\infty}\left(B, \mathscr{H}_{p}\right)$, called the relative Bergman projection on $\mathscr{H}_{p}$ over $\pi: M \rightarrow B$. On the other hand, for any $K_{1, p}(\cdot, \cdot), K_{2, p}(.,.) \in \mathscr{C}^{\infty}\left(M \times_{B} M, E_{p} \bigotimes_{B} E_{p}^{*}\right)$, we write $K_{1, p} K_{2, p}(.,.) \in \mathscr{C}^{\infty}\left(M \times_{B} M, E_{p} \bigotimes_{B} E_{p}^{*}\right)$ for the kernel defined for any $b \in B, x, y \in X_{b}$ by

$$
K_{1, p} K_{2, p}(x, y)=\int_{X} K_{1, p}(x, w) K_{2, p}(w, y) d v_{X_{b}}(w) .
$$

For any $v \in \mathscr{C}^{\infty}(B, T B)$, consider $\nabla_{v^{H}}^{E_{p}}$ as a first order differential operator acting on $\mathscr{C}^{\infty}\left(M, E_{p}\right)$. This can be seen as the contraction with $v \in \mathscr{C}^{\infty}(B, T B)$ of a connection on the infinite dimensional vector bundle of fibrewise smooth sections. The $L^{2}$-Hermitian connection on $\mathscr{H}_{p}$ over $B$ is defined for any $v \in \mathscr{C}^{\infty}(B, T B)$ by

$$
\nabla_{v}^{\mathscr{H}_{p}}=P_{p} \nabla_{v^{H}}^{E_{p}} P_{p} .
$$

By an argument of [17, Th.1.14], $\nabla_{v^{H}}^{E_{p}}$ preserves the $L^{2}$-Hermitian product (2.8), so that $\nabla^{\mathscr{H}_{p}}$ is a Hermitian connection on $\left(\mathscr{H}_{p}, h^{\mathscr{H}_{p}}\right)$.

A prequantized fibration is called holomorphic if $\pi: M \rightarrow B$ is a holomorphic submersion between complex manifolds, and if $\left(L, h^{L}\right),\left(E, h^{E}\right)$ are holomorphic Hermitian bundles equipped with their Chern connections $\nabla^{L}, \nabla^{E}$, which are the unique Hermitian connections preserving the holomorphic structure. The relative complex structure $J \in \operatorname{End}(T X)$ induced by the natural holomorphic structure in the fibres is then compatible with $\omega$, and this makes $\pi: M \rightarrow B$ into a Kähler fibration in the sense of [17, Def.1.4]. Set $\Psi=-\sqrt{-1} \Lambda_{\omega} R^{E}$ in (2.10), where $\Lambda_{\omega}$ denotes the contraction by $\omega$. For a proof of the following proposition, we refer to [41, §1.4.3], [22, Th.1.1], [20, Th.3.2] and the references therein. 
Proposition 2.3. Let $\pi: M \rightarrow B$ be a holomorphic prequantized fibration with $B$ compact. Then there exists $p_{0} \in \mathbb{N}$ such that for any $p \geqslant p_{0}$ and $b \in B$, the space $\mathscr{H}_{p, b}$ coincides with the space of holomorphic sections of $E_{p, b}$ inside $\mathscr{C}^{\infty}\left(X_{b}, E_{p, b}\right)$, and the bundle $\mathscr{H}_{p}$ over $B$ has a natural holomorphic structure, for which the $L^{2}$-Hermitian connection $\nabla^{\mathscr{H}_{p}}$ on $\mathscr{H}_{p}$ is the Chern connection of $\left(\mathscr{H}_{p}, h^{\mathscr{H}_{p}}\right)$.

Let us finally set the following convention : for any $k \in \mathbb{N}$, if $A, B \in \operatorname{End}\left(\mathbb{R}^{k}\right)$ are symmetric matrices with $A$ positive, we define $\operatorname{det} \frac{1}{2}(A+\sqrt{-1} B)$ to be the square root of $\operatorname{det}(A+\sqrt{-1} B)$ determined by analytic continuation along the path $t \mapsto A+t \sqrt{-1} B$ for $t \in[0,1]$.

\section{$2.2 \quad$ Bergman kernels}

In this section, we will use systematically the fact that all the estimates described in [41, Chap.4, §8.3.2] are uniform with respect to parameters. Let $\pi: M \rightarrow B$ be a prequantized fibration equipped with a relative almost complex structure $J \in \operatorname{End}(T X)$ compatible with $\omega$. We assume $B$ compact, and fix $p_{0} \in \mathbb{N}$ as in Theorem 2.2 for $U=B$.

For any $m \in \mathbb{N}$ and all $p \in \mathbb{N}^{*}$, we denote by $|\cdot| \mathscr{C}^{m}$ the $\mathscr{C}^{m}$-norm on $E_{p} \bigotimes_{B} E_{p}^{*}$ induced by $h^{L}, h^{E}, \nabla^{L}, \nabla^{E}$. Recall that $X_{b}$ denotes the fibre of $\pi: M \rightarrow B$ at $b \in B$, and $g_{b}^{T X}$ the restriction of $g^{T X}$ to $X_{b}$. Let $d^{X_{b}}(\cdot, \cdot)$ be the Riemannian distance on $\left(X_{b}, g_{b}^{T X}\right)$. The following result comes essentially from $[39,(2.5)]$, built on arguments of $[41, \S 8.3 .3],[42, \S 1.1]$.

Proposition 2.4. For any $m, k \in \mathbb{N}, \varepsilon>0$ and $\theta \in] 0,1[$, there is $C>0$ such that for all $p \in \mathbb{N}^{*}, b \in B$ and $x, x^{\prime} \in X_{b}$ satisfying $d^{X_{b}}\left(x, x^{\prime}\right)>\varepsilon p^{-\frac{\theta}{2}}$, the following estimate holds,

$$
\left|P_{p}\left(x, x^{\prime}\right)\right|_{\mathscr{C}^{m}} \leqslant C p^{-k}
$$

Proposition 2.4 shows that the study of the asymptotics in $p \in \mathbb{N}^{*}$ of the Bergman kernel localizes around the diagonal of the fibres. To describe asymptotic estimates for the Bergman kernel near the diagonal, we will need the following definition.

Definition 2.5. Let $\psi$ be a smooth map from the total space of $T X$ to $M$, and for any $x \in M$, write $\psi_{x}: T_{x} X \rightarrow M$ for the restriction of $\psi$ to $T_{x} M$. We say that $\psi$ is a smooth family of vertical charts if for any $x \in M$, the image of $\psi_{x}$ is included in $X_{\pi(x)}$, such that $\psi_{x}(0)=x$ and its differential $d \psi_{x, 0}: T_{x} X \rightarrow T_{x} M$ at 0 induces the canonical injection of $T_{x} X$ into $T_{x} M$.

Using partitions of unity, it is easy to see that smooth families of vertical charts always exist. We fix one of them for the rest of the section. For any $x \in M$ and $\varepsilon>0$, let $|\cdot|_{x}$ be the norm of $\left(T_{x} X, g^{T_{x} X}\right)$ and let $B^{T_{x} X}(0, \varepsilon)$ be the open ball in $T_{x} X$ of radius $\varepsilon$. Choose $\varepsilon_{0}>0$ so small that for any $x \in M$, the map $\psi_{x}$ induces a diffeomorphism between $B^{T_{x} X}\left(0, \varepsilon_{0}\right)$ and a neighborhood $U_{x}$ of $x$ in $X_{\pi(x)}$. Identify $L, E$ over $U_{x}$ with $L_{x}, E_{x}$ through parallel transport with respect to $\nabla^{L}, \nabla^{E}$ along radial lines of $B^{T_{x} X}\left(0, \varepsilon_{0}\right)$ in the chart induced by $\psi_{x}$. Pick a unit section $e_{x} \in L_{x}$ and 
use it to identify $L_{x}$ with $\mathbb{C}$. In this way, the vector bundle $E_{p}$ is identified with $E_{x}$ over $U_{x}$ for all $p \in \mathbb{N}^{*}$, and this identification can be made smoothly in $x \in M$. Under the natural isomorphism $\operatorname{End}\left(L^{p}\right) \simeq \mathbb{C}$, our formulas do not depend on this identification.

For any $p \in \mathbb{N}^{*}$ and any kernel $K_{p}(\cdot, \cdot) \in \mathscr{C}^{\infty}\left(M \times_{B} M, E_{p} \bigotimes_{B} E_{p}^{*}\right)$, we write $K_{p, x}\left(Z, Z^{\prime}\right) \in \operatorname{End}\left(E_{x}\right)$ for its image in this trivialization evaluated at $Z, Z^{\prime} \in B^{T_{x} X}\left(0, \varepsilon_{0}\right)$. Note that $K_{p, x}$ is a section of the pullback bundle $\pi_{0}^{*} \operatorname{End}(E)$ over an open set of the fibred product $\pi_{0}: T X \times_{M} T X \rightarrow M$ of the total space of $T X$ with itself over $M$. On the other hand, if $\mathscr{K} \in \pi_{0}^{*} \operatorname{End}(E)$ is such a section and $F_{x}\left(Z, Z^{\prime}\right) \in \operatorname{End}\left(E_{x}\right)$ is polynomial in $Z, Z^{\prime} \in T_{x} X$ for any $x \in M$, we write

$$
F \mathscr{K}_{x}\left(Z, Z^{\prime}\right)=F_{x}\left(Z, Z^{\prime}\right) \mathscr{K}_{x}\left(Z, Z^{\prime}\right)
$$

Recalling that two Riemannian metrics induce equivalent distances in a continuous way with respect to parameters, we can precise Proposition 2.4 in the following way.

Corollary 2.6. For any $m, k \in \mathbb{N}, \varepsilon>0$ and $\theta \in] 0,1[$, there $i s C>0$ such that for all $p \in \mathbb{N}^{*}$ and $x \in X, Z, Z^{\prime} \in B^{T_{x} X}\left(0, \varepsilon_{0}\right)$ such that $\left|Z-Z^{\prime}\right|_{x}>\varepsilon p^{-\frac{\theta}{2}}$, the following estimate holds,

$$
\left|P_{p, x}\left(Z, Z^{\prime}\right)\right|_{\mathscr{C}^{m}} \leqslant C p^{-k}
$$

We use the following explicit local model for the Bergman kernel from [43, (3.25)], for any $x \in M, Z, Z^{\prime} \in T_{x} X$,

$$
\mathscr{P}_{x}\left(Z, Z^{\prime}\right)=\exp \left(-\frac{\pi}{2}\left|Z-Z^{\prime}\right|_{x}^{2}-\pi \sqrt{-1} \omega_{x}^{X}\left(Z, Z^{\prime}\right)\right)
$$

Let $|\cdot|_{\mathscr{C}^{m}(M)}$ denote the $\mathscr{C}^{m}$-norm on $\pi_{0}^{*} \operatorname{End}(E)$ over $T X \times_{M} T X$ induced by $h^{E}$ and by derivation by $\nabla^{\pi_{0}^{*} \operatorname{End}(E)}$ in the direction of $M$ via the Levi-Civita connection. We can now state the following fundamental result on the near diagonal expansion of the Bergman kernel, which was first established in [29, Th.4.18'] for $\operatorname{spin}^{c}$ Dirac operators, hence in particular in the Kähler case. In this form, it comes essentially from [39, Th.2.1].

Proposition 2.7. There is a family $\left\{J_{r, x}\left(Z, Z^{\prime}\right) \in \operatorname{End}\left(E_{x}\right)\right\}_{r \in \mathbb{N}}$ of polynomials in $Z, Z^{\prime} \in T_{x} X$ of the same parity as $r$ and smooth in $x \in M$, such that for any $\varepsilon>$ $0, k, m, m^{\prime} \in \mathbb{N}$ and $\left.\delta \in\right] 0,1[$, there is $\theta \in] 0,1[$ and $C>0$ such that for all $x \in M, p \in$ $\mathbb{N}^{*},|Z|,\left|Z^{\prime}\right|<\varepsilon_{0} p^{-\frac{\theta}{2}}$,

$$
\begin{aligned}
\sup _{|\alpha|+\left|\alpha^{\prime}\right| \leqslant m} \mid \frac{\partial^{\alpha}}{\partial Z^{\alpha}} \frac{\partial^{\alpha^{\prime}}}{\partial Z^{\prime \alpha^{\prime}}}\left(p^{-n} P_{p, x}\left(Z, Z^{\prime}\right)\right. & \\
& \left.-\sum_{r=0}^{k-1} J_{r} \mathscr{P}_{x}\left(\sqrt{p} Z, \sqrt{p} Z^{\prime}\right) p^{-\frac{r}{2}}\right)\left.\right|_{\mathscr{C}^{m^{\prime}}(M)} \leqslant C p^{-\frac{k-m}{2}+\delta} .
\end{aligned}
$$

Furthermore, for all $x \in M, Z, Z^{\prime} \in T_{x} X$, we have $J_{0, x}\left(Z, Z^{\prime}\right)=\operatorname{Id}_{E_{x}}$. 
We will often consider expansions of this type in the sequel. To this end, we introduce the following notation.

Notation 2.8. For any family $\left\{K_{p}(., .) \in \mathscr{C}^{\infty}\left(M \times_{B} M, E_{p} \bigotimes_{B} E_{p}^{*}\right)\right\}_{p \in \mathbb{N}^{*}}$, we write

$$
p^{-n} K_{p, x}\left(Z, Z^{\prime}\right) \cong \sum_{r=0}^{\infty} \mathcal{Q}_{r} \mathscr{K}_{x}\left(\sqrt{p} Z, \sqrt{p} Z^{\prime}\right) p^{-\frac{r}{2}}+\mathscr{O}\left(p^{-\infty}\right)
$$

for $\mathscr{K}_{x}\left(Z, Z^{\prime}\right) \in \operatorname{End}\left(E_{x}\right)$ smooth in $x \in M, Z, Z^{\prime} \in T_{x} X$, and $\left\{\mathcal{Q}_{r, x}\left(Z, Z^{\prime}\right) \in \operatorname{End}\left(E_{x}\right)\right\}_{r \in \mathbb{N}}$ a family of polynomials in $Z, Z^{\prime} \in T_{x} X$, smooth in $x \in M$, if there exists $\varepsilon>0$ such that for any $k, m, m^{\prime} \in \mathbb{N}$ and $\left.\theta \in\right] 0,1[$, there is $C>0$, such that for all $x \in M$ and $Z, Z^{\prime} \in B^{T_{x} X}\left(0, \varepsilon_{0}\right)$ with $\left|Z-Z^{\prime}\right|_{x}>\varepsilon p^{-\frac{\theta}{2}}$,

$$
\left|K_{p, x}\left(Z, Z^{\prime}\right)\right|_{\mathscr{C}^{m}} \leqslant C p^{-k}
$$

and such that for all $\delta \in] 0,1[$, there is $\theta \in] 0,1[$ and $C>0$ such that for all $x \in$ $M,|Z|,\left|Z^{\prime}\right|<\varepsilon p^{-\frac{\theta}{2}}$,

$$
\begin{aligned}
\sup _{|\alpha|+\left|\alpha^{\prime}\right| \leqslant m} \mid \frac{\partial^{\alpha}}{\partial Z^{\alpha}} \frac{\partial^{\alpha^{\prime}}}{\partial Z^{\prime \alpha^{\prime}}}\left(p^{-n} K_{p, x}\left(Z, Z^{\prime}\right)\right. & \\
& \left.\quad-\sum_{r=0}^{k-1} \mathcal{Q}_{r} \mathscr{K}_{x}\left(\sqrt{p} Z, \sqrt{p} Z^{\prime}\right) p^{-\frac{r}{2}}\right)\left.\right|_{\mathscr{C}^{m^{\prime}}(M)} \leqslant C p^{-\frac{k-m}{2}+\delta} .
\end{aligned}
$$

For any $f \in \mathscr{C}^{\infty}(M, \operatorname{End}(E))$, the Berezin-Toeplitz quantization of $f$ is the family of endomorphisms of $\mathscr{H}_{p}$ for all $p \in \mathbb{N}^{*}$, defined for any $b \in B$ by the formula

$$
P_{p, b} f P_{p, b}: \mathscr{C}^{\infty}\left(X_{b}, E_{p, b}\right) \rightarrow \mathscr{C}^{\infty}\left(X_{b}, E_{p, b}\right)
$$

where $f$ denotes the operator of pointwise multiplication by $f$. By (2.17), this operator admits a smooth Schwarz kernel with respect to $d v_{X_{b}}$. The following asymptotic expansion in $p \in \mathbb{N}^{*}$ is a consequence of Corollary 2.6 and Proposition 2.7. It was first established in [41, Lem.4.6] for $\operatorname{spin}^{c}$ Dirac operators, hence in particular in the Kähler case. In this form, it comes essentially from [35, Lem.3.3].

Lemma 2.9. There is a family $\left\{\mathcal{Q}_{r, x}(f)\left(Z, Z^{\prime}\right) \in \operatorname{End}\left(E_{x}\right)\right\}_{r \in \mathbb{N}}$ of polynomials in $Z, Z^{\prime} \in T_{x} X$ of the same parity as $r$, smooth in $x \in M$, such that

$$
p^{-n}\left(P_{p, b} f P_{p, b}\right)\left(Z, Z^{\prime}\right) \cong \sum_{r=0}^{\infty} \mathcal{Q}_{r}(f) \mathscr{P}_{x}\left(\sqrt{p} Z, \sqrt{p} Z^{\prime}\right) p^{-\frac{r}{2}}+\mathscr{O}\left(p^{-\infty}\right)
$$

Furthermore, for all $x \in M, Z, Z^{\prime} \in T_{x} X$, we have $\mathcal{Q}_{0, x}(f)\left(Z, Z^{\prime}\right)=f(x)$. 


\subsection{Trivialization of fibrations}

In Section 3.3, we will be mainly concerned with the study of prequantized fibrations restricted over paths parametrized by $t \in[0,1]$. Keeping this in mind, we consider in this section the case of a prequantized fibration $\pi: M \rightarrow[0,1]$.

Let $\partial_{t}$ be the canonical vector field of $[0,1]$ and recall that $\partial_{t}^{H}$ denotes its horizontal lift to $T^{H} M$ in $T M$. Set $X:=\pi^{-1}(0)$. Then there is a unique diffeomorphism $\tau$ between $[0,1] \times X$ and $M$ such that for any $t \in[0,1]$ and $x_{0} \in X$,

$$
\tau\left(0, x_{0}\right)=x_{0} \quad \text { and } \quad \frac{\partial}{\partial t} \tau\left(t, x_{0}\right)=\partial_{t, x_{0}}^{H} .
$$

The fibration coincides via $\tau$ with the first projection $\pi:[0,1] \times X \rightarrow X$. By (1.1) and (2.1), for any vertical vector field $v \in \mathscr{C}^{\infty}(M, T X)$, we have

$$
R^{L}\left(\partial_{t}^{H}, v\right)=0 .
$$

This shows parallel transport with respect to $\nabla^{L}$ along horizontal paths of $[0,1] \times X$ via $\tau$ identifies $\left(L, h^{L}, \nabla^{L}\right)$ over $[0,1] \times X$ with the pullback of a fixed Hermitian line bundle with connection over $X$, which we still denote by $\left(L, h^{L}, \nabla^{L}\right)$. In particular, we have

$$
\nabla_{\partial_{t}^{H}}^{L}=\frac{\partial}{\partial t} \quad \text { and } \quad\left[\frac{\partial}{\partial t}, \nabla^{L}\right]=0
$$

By (1.1), we deduce that $\omega$ is identified via $\tau$ with the pullback of a fixed symplectic form $\omega^{X} \in \Omega^{2}(X, \mathbb{R})$ over $X$, so that the restriction of $\omega$ to $X_{t} \simeq X$ does not depend on $t \in[0,1]$. Then the volume form on the fibre $X$ induced by $\omega$ as in $(2.7)$ does not depend on $t \in[0,1]$ either. We write $J_{t}, g_{t}^{T X}, E_{t}$ for the restriction of $J, g^{T X}, E$ to $X \simeq X_{t}$ over $t \in[0,1]$. Let $\tau_{t}^{E}: E_{0} \rightarrow E_{t}$ be the vector bundle isomorphism induced by parallel transport with respect to $\nabla^{E}$ along horizontal curves of $[0,1] \times X$.

Let $\psi$ be a smooth family of vertical charts over $\pi:[0,1] \times X \rightarrow[0,1]$ such that its restriction $\psi_{t}: T X \rightarrow X$ over the fibre at $t \in[0,1]$ does not depend on $t$. Fix $x_{0} \in X$, and consider the trivialization around $x_{0}$ as in Section 2.2. Identify $T_{x_{0}} X$ with $\mathbb{R}^{2 n}$ using an orthonormal basis $\left\{e_{j}\right\}_{j=1}^{n}$ of $\left(T_{x_{0}} X, g_{0}^{T X}\right)$ such that

$$
J_{0} e_{2 j}=e_{2 j+1} \quad \text { and } \quad J_{0} e_{2 j+1}=-e_{2 j} .
$$

Then $\omega_{x_{0}}^{X}$ induces the standard symplectic form $\Omega$ on $\mathbb{R}^{2 n}$ in this identification. Write $|\cdot|_{t}$ for the norm on $\mathbb{R}^{2 n}$ induced by $g_{t}^{T X}$, so that in particular $|\cdot|_{0}$ is the standard norm of $\mathbb{R}^{2 n}$. Then the local model (2.22) of the Bergman kernel on $\left(T_{x_{0}} X, J_{t}\right)$ becomes

$$
\left.\mathscr{P}_{t, x_{0}}\left(Z, Z^{\prime}\right)=\exp \left(-\frac{\pi}{2}\left|Z-Z^{\prime}\right|_{t}^{2}-\pi \sqrt{-1} \Omega\left(Z, Z^{\prime}\right)\right)\right),
$$

for all $Z, Z^{\prime} \in \mathbb{R}^{2 n}$ and $t \in[0,1]$.

We end this section with the following definition, which generalizes the setting described above. 
Definition 2.10. A prequantized fibration $\pi: M \rightarrow B$ is said to be tautological if there is a fibration map $M \simeq B \times X$ such that the associated line bundle $\left(L, h^{L}, \nabla^{L}\right)$ over $M$ is the pullback of a Hermitian line bundle with connection over $X$ by the second projection $\pi_{2}: B \times X \rightarrow X$.

If $J \in \operatorname{End}(T X)$ is a relative compatible almost complex structure over a tautological fibration, we write $J_{b}$ for the restriction of $J$ to $X \simeq X_{b}$ over $b \in B$. Then $J$ can be seen as a family of compatible almost complex structures on a fixed symplectic manifold $\left(X, \omega^{X}\right)$, depending smoothly on $b \in B$.

\section{Toeplitz operators}

In Section 3.1, we study the local model for the composition of Bergman kernels associated with different complex structures via the identifications of Section 2.3. In Section 3.2, we study the corresponding generalization of a Toeplitz operator. In Section 3.3, we use these results to show that the parallel transport in the quantum bundle over a path of complex structures is a such a Toeplitz operator, proving Theorem 1.1.

\subsection{Local model}

Let $Z:=\left(Z_{1}, \ldots, Z_{2 n}\right) \in \mathbb{R}^{2 n}$ denote the real coordinates of $\mathbb{R}^{2 n}$. Let $\langle\cdot, \cdot\rangle$ be the canonical scalar product on $\mathbb{R}^{2 n}$, and write $|\cdot|$ for the associated norm. We write $J_{0} \in \operatorname{End}\left(\mathbb{R}^{2 n}\right)$ for the complex structure defined for all $1 \leqslant j \leqslant n$ by

$$
J_{0} \frac{\partial}{\partial Z_{2 j}}=-\frac{\partial}{\partial Z_{2 j-1}} \quad \text { and } \quad J_{0} \frac{\partial}{\partial Z_{2 j-1}}=\frac{\partial}{\partial Z_{2 j}} .
$$

Then $\langle\cdot, \cdot\rangle$ is $J_{0}$-invariant, and $\Omega(\cdot, \cdot)=\left\langle J_{0} \cdot, \cdot\right\rangle$ defines a $J_{0}$-invariant antisymmetric non-degenerate form on $\mathbb{R}^{2 n}$, called the canonical symplectic form.

Let now $J_{t} \in \operatorname{End}\left(\mathbb{R}^{2 n}\right)$ satisfying $J_{t}^{2}=-\operatorname{Id}_{\mathbb{R}^{2 n}}$ be a smooth one-parameter family of compatible complex structures on $\mathbb{R}^{2 n}$, so that $\Omega$ is $J_{t}$-invariant and the formula $\langle\cdot, \cdot\rangle_{t}=\Omega\left(\cdot, J_{t} \cdot\right)$ defines a scalar product on $\mathbb{R}^{2 n}$, for all $t \in \mathbb{R}$. Note that $\langle\cdot, \cdot\rangle_{t}$ and $\langle\cdot, \cdot\rangle$ are related by

$$
\langle\cdot, \cdot\rangle_{t}=\left\langle\left(-J_{0} J_{t}\right) \cdot, \cdot\right\rangle
$$

In particular $-J_{0} J_{t} \in \operatorname{End}\left(\mathbb{R}^{2 n}\right)$ is positive symmetric, as well as its inverse $-J_{t} J_{0}$. We write $|\cdot|_{t}$ for the norm induced on $\mathbb{R}^{2 n}$ by $\langle\cdot, \cdot\rangle_{t}$.

Recall the local model (2.33) for the Bergman kernel in $\mathbb{R}^{2 n}$ associated to $J_{t}$ for any $t \in \mathbb{R}$, which is given for any $Z, Z^{\prime} \in \mathbb{R}^{2 n}$ by

$$
\begin{aligned}
\mathscr{P}_{t}\left(Z, Z^{\prime}\right) & \left.=\exp \left(-\frac{\pi}{2}\left|Z-Z^{\prime}\right|_{t}^{2}-\pi \sqrt{-1} \Omega\left(Z, Z^{\prime}\right)\right)\right) \\
& \left.=\exp \left(-\frac{\pi}{2}\left\langle\left(-J_{0} J_{t}\right)\left(Z-Z^{\prime}\right),\left(Z-Z^{\prime}\right)\right\rangle-\pi \sqrt{-1} \Omega\left(Z, Z^{\prime}\right)\right)\right) .
\end{aligned}
$$


In particular, we have $\mathscr{P}_{t}\left(Z, Z^{\prime}\right)=\overline{\mathscr{P}_{t}\left(Z^{\prime}, Z\right)}$. Note that the canonical Lebesgue measure $d Z$ of $\mathbb{R}^{2 n}$ is induced by the Liouville form of $\Omega$, and thus corresponds to the Riemannian volume form of $\langle\cdot, \cdot\rangle_{t}$ for all $t \in[0,1]$. For any $t \in \mathbb{R}$, let $\mathscr{H}_{t} \subset L^{2}\left(\mathbb{R}^{2 n}\right)$ be defined by

$$
\mathscr{H}_{t}:=\left\{f \in L^{2}\left(\mathbb{R}^{2 n}\right) \mid Z \mapsto f(Z) e^{\frac{\pi}{2}|Z|^{2}} \text { is holomorphic for } J_{t}\right\}
$$

Then as explained in $[42, \S 1.4], \mathscr{P}_{t}(\cdot, \cdot)$ is the Schwartz kernel with respect to $d Z$ of the orthogonal projection $\mathscr{P}_{t}: L^{2}\left(\mathbb{R}^{n}\right) \rightarrow \mathscr{H}_{t}$, and in particular we have $\mathscr{P}_{t} \mathscr{P}_{t}=\mathscr{P}_{t}$. The Schwartz kernel of the composition $\mathscr{P}_{t} \mathscr{P}_{0}$ is given for any $Z, Z^{\prime} \in \mathbb{R}^{2 n}$ by the formula

$$
\mathscr{P}_{t} \mathscr{P}_{0}\left(Z, Z^{\prime}\right)=\int_{\mathbb{R}^{2 n}} \mathscr{P}_{t}(Z, \widetilde{Z}) \mathscr{P}_{0}\left(\widetilde{Z}, Z^{\prime}\right) d \widetilde{Z}
$$

Let $\mathbb{C}^{2 n}=V_{t}^{(1,0)} \oplus V_{t}^{(0,1)}$ be the splitting of $\mathbb{C}^{2 n}=\mathbb{R}^{2 n} \otimes_{\mathbb{R}} \mathbb{C}$ into the eigenspaces of $J_{t}$ corresponding to the eigenvalues $\sqrt{-1}$ and $-\sqrt{-1}$. The corresponding projections $P_{t}^{(1,0)}, P_{t}^{(0,1)}$ from $\mathbb{C}^{2 n}$ to $V_{t}^{(1,0)}, V_{t}^{(0,1)}$ are given by the formulas

$$
P_{t}^{(1,0)}=\frac{1-\sqrt{-1} J_{t}}{2} \text { and } P_{t}^{(0,1)}=\frac{1+\sqrt{-1} J_{t}}{2} .
$$

For any $t \in \mathbb{R}$, define the symmetric positive endomorphisms

$$
A_{t}^{0}=\left(\frac{\mathrm{Id}-J_{0} J_{t}}{2}\right)^{-1} \quad \text { and } \quad A_{0}^{t}=\left(\frac{\mathrm{Id}-J_{t} J_{0}}{2}\right)^{-1} .
$$

and set

$$
\Pi_{t}^{0}=A_{t}^{0} P_{0}^{(1,0)} \quad \text { and } \quad \Pi_{0}^{t}=A_{0}^{t} P_{t}^{(1,0)} .
$$

Then we have the following identities,

$$
\begin{array}{ccc}
\Pi_{t}^{0} P_{t}^{(1,0)}=P_{t}^{(1,0)} & \text { and } \quad \Pi_{0}^{t} P_{0}^{(1,0)}=P_{0}^{(1,0)} \\
P_{t}^{(1,0)} \Pi_{t}^{0}=\Pi_{t}^{0} & \text { and } & P_{0}^{(1,0)} \Pi_{0}^{t}=\Pi_{0}^{t},
\end{array}
$$

which, together with (3.8), show that $\Pi_{t}^{0}, \Pi_{0}^{t} \in \operatorname{End}\left(\mathbb{C}^{2 n}\right)$ are precisely the projection operators onto $V_{t}^{(1,0)}, V_{0}^{(1,0)}$ with kernel $V_{0}^{(0,1)}, V_{t}^{(0,1)}$. In particular, they induce a splitting

$$
\mathbb{C}^{2 n}=V_{t}^{(1,0)} \oplus V_{0}^{(0,1)}
$$

for all $t \in \mathbb{R}$. Their complex conjugates $\overline{\Pi_{t}^{0}}, \overline{\Pi_{0}^{t}} \in \operatorname{End}\left(\mathbb{C}^{2 n}\right)$ are the projection operators onto $V_{t}^{(0,1)}, V_{0}^{(0,1)}$ with kernel $V_{0}^{(1,0)}, V_{t}^{(1,0)}$, and induce a splitting of $\mathbb{C}^{2 n}$ which is complex conjugate to (3.10). Note that these projections all have positive symmetric real part given by $(3.7)$.

The following result is an explicit computation of the kernel (3.5), which is going to be our local model in the next section. 
Lemma 3.1. For any $Z, Z^{\prime} \in \mathbb{R}^{2 n}$, the following formula holds

$$
\mathscr{P}_{t} \mathscr{P}_{0}\left(Z, Z^{\prime}\right)=\operatorname{det}\left(A_{t}^{0}\right)^{\frac{1}{2}} \exp \left(-\pi\left[\left\langle\Pi_{0}^{t}\left(Z-Z^{\prime}\right),\left(Z-Z^{\prime}\right)\right\rangle+\sqrt{-1} \Omega\left(Z, Z^{\prime}\right)\right]\right) .
$$

Furthermore, for any $F(Z) \in \mathbb{C}[Z]$ homogeneous, there exists $\mathcal{Q}(F)(Z) \in \mathbb{C}[Z]$ of the same parity such that for any $Z, Z^{\prime} \in \mathbb{R}^{2 n}$,

$$
\int_{\mathbb{R}^{2 n}} \mathscr{P}_{t}(Z, \widetilde{Z}) F(\widetilde{Z}) \mathscr{P}_{0}\left(\widetilde{Z}, Z^{\prime}\right) d \widetilde{Z}=\mathcal{Q}(F)(Z) \mathscr{P}_{t} \mathscr{P}_{0}\left(Z, Z^{\prime}\right) .
$$

Finally, for any $B \in \operatorname{End}\left(\mathbb{C}^{2 n}\right)$ and $Z, Z^{\prime} \in \mathbb{R}^{2 n}$, the following formulas hold,

$$
\begin{aligned}
\int_{\mathbb{R}^{2 n}} \mathscr{P}_{t}(Z, \tilde{Z}) & \langle B(Z-\widetilde{Z}),(Z-\widetilde{Z})\rangle \mathscr{P}_{0}\left(\widetilde{Z}, Z^{\prime}\right) d \widetilde{Z} \\
= & \left(\left\langle B \overline{\Pi_{t}^{0}}\left(Z-Z^{\prime}\right), \overline{\Pi_{t}^{0}}\left(Z-Z^{\prime}\right)\right\rangle+\frac{1}{2 \pi} \operatorname{Tr}\left[A_{t}^{0} B\right]\right) \mathscr{P}_{t} \mathscr{P}_{0}\left(Z, Z^{\prime}\right) \\
\int_{\mathbb{R}^{2 n}} \mathscr{P}_{t}(Z, \widetilde{Z}) & \left\langle B\left(Z^{\prime}-\widetilde{Z}\right),\left(Z^{\prime}-\widetilde{Z}\right)\right\rangle \mathscr{P}_{0}\left(\widetilde{Z}, Z^{\prime}\right) d \widetilde{Z} \\
= & \left(\left\langle B \Pi_{0}^{t}\left(Z-Z^{\prime}\right), \Pi_{0}^{t}\left(Z-Z^{\prime}\right)\right\rangle+\frac{1}{2 \pi} \operatorname{Tr}\left[A_{0}^{t} B\right]\right) \mathscr{P}_{t} \mathscr{P}_{0}\left(Z, Z^{\prime}\right) .
\end{aligned}
$$

Proof. Let $Z, Z^{\prime} \in \mathbb{R}^{2 n}$ be fixed, and recall that $\Omega(\cdot, \cdot)=\left\langle J_{0} \cdot, \cdot\right\rangle$. Through the change of variable $\widetilde{Z} \mapsto \widetilde{Z}+Z$ and using (3.5), we get

$$
\begin{aligned}
& \mathscr{P}_{t} \mathscr{P}_{0}\left(Z, Z^{\prime}\right)=\int_{\mathbb{R}^{2 n}} \exp \left(-\frac{\pi}{2}\left[\left\langle\left(-J_{0} J_{t}\right) \widetilde{Z}, \widetilde{Z}\right\rangle+\left|\widetilde{Z}+\left(Z-Z^{\prime}\right)\right|^{2}\right.\right. \\
&+\left.\left.2 \sqrt{-1} \Omega(Z, \widetilde{Z})+2 \sqrt{-1} \Omega\left(\widetilde{Z}+Z, Z^{\prime}\right)\right]\right) d \widetilde{Z} \\
&=\int_{\mathbb{R}^{2 n}} \exp \left(-\frac{\pi}{2}\left[\left\langle\left(\mathrm{Id}-J_{0} J_{t}\right) \widetilde{Z}, \widetilde{Z}\right\rangle+4\left\langle\widetilde{Z}, P_{0}^{(0,1)}\left(Z-Z^{\prime}\right)\right\rangle\right]\right) d \widetilde{Z} \\
& \exp \left(-\frac{\pi}{2}\left[\left|Z-Z^{\prime}\right|^{2}+2 \sqrt{-1} \Omega\left(Z, Z^{\prime}\right)\right]\right) .
\end{aligned}
$$

By (3.7) and the classical formula for Gaussian integrals, we then get

$$
\begin{aligned}
\mathscr{P}_{t} \mathscr{P}_{0}\left(Z, Z^{\prime}\right)=\operatorname{det}\left(A_{t}^{0}\right)^{\frac{1}{2}} \exp \left(\pi\left\langle A_{t}^{0} P_{0}^{(0,1)}\left(Z-Z^{\prime}\right), P_{0}^{(0,1)}\left(Z-Z^{\prime}\right)\right\rangle\right) \\
\exp \left(-\frac{\pi}{2}\left[\left|Z-Z^{\prime}\right|^{2}+2 \sqrt{-1} \Omega\left(Z, Z^{\prime}\right)\right]\right) .
\end{aligned}
$$

From (3.7), we know that $-J_{0} A_{t}^{0} J_{0}=\left(-J_{0} J_{t}\right) A_{t}^{0}=A_{0}^{t}$, and in particular, we get the identities

$$
\begin{aligned}
A_{t}^{0}+A_{0}^{t} & =\left(1+\left(-J_{0} J_{t}\right)\right) A_{t}^{0}=2 \operatorname{Id}_{\mathbb{R}^{2 n}} \\
A_{t}^{0} J_{0} & =J_{0} A_{0}^{t}=A_{0}^{t} J_{t}, \\
P_{0}^{(1,0)} A_{t}^{0} P_{0}^{(1,0)} & =\frac{1}{4}\left(A_{t}^{0}-A_{0}^{t}-\sqrt{-1} J_{0} A_{t}^{0}+\sqrt{-1} A_{t}^{0} J_{0}\right) .
\end{aligned}
$$


Recall that $A_{t}^{0}$ and $A_{0}^{t}$ are symmetric. Using (3.6), (3.8) and these identities, we can rewrite (3.15) into

$$
\begin{aligned}
& \mathscr{P}_{t} \mathscr{P}_{0}\left(Z, Z^{\prime}\right)=\operatorname{det}\left(A_{t}^{0}\right)^{\frac{1}{2}} \exp \left(-\frac{\pi}{4}\left\langle\left(A_{0}^{t}-A_{t}^{0}\right)\left(Z-Z^{\prime}\right),\left(Z-Z^{\prime}\right)\right\rangle\right) \\
& \exp \left(-\frac{\pi}{4}\left[-2 \sqrt{-1}\left\langle A_{t}^{0} J_{0}\left(Z-Z^{\prime}\right), Z-Z^{\prime}\right\rangle\right]\right) \\
& \exp \left(-\frac{\pi}{4}\left[\left\langle\left(A_{0}^{t}+A_{t}^{0}\right)\left(Z-Z^{\prime}\right), Z-Z^{\prime}\right\rangle+4 \sqrt{-1} \Omega\left(Z, Z^{\prime}\right)\right]\right) \\
& =\operatorname{det}\left(A_{t}^{0}\right)^{\frac{1}{2}} \exp \left(-\pi\left[\left\langle\Pi_{0}^{t}\left(Z-Z^{\prime}\right),\left(Z-Z^{\prime}\right)\right\rangle+\sqrt{-1} \Omega\left(Z, Z^{\prime}\right)\right]\right) .
\end{aligned}
$$

This implies (3.11). The computations leading to (3.12) and (3.13) are analogous to (3.14)-(3.17), applying the classical formula for the integral of a Gaussian function multiplied by a polynomial. Note that the second equality of (3.13) can be deduced from the first using $\mathscr{P}_{t}\left(Z, Z^{\prime}\right)=\overline{\mathscr{P}_{t}\left(Z^{\prime}, Z\right)}$ and exchanging the roles of 0 and $t$.

We recover from (3.11) with $t=0$ the identity $\mathscr{P}_{0} \mathscr{P}_{0}=\mathscr{P}_{0}$ characterizing projection operators. For $t=0$, the formulas (3.12) and (3.13) are consequences of [43, §2]. For any $t \in \mathbb{R}$, set

$$
\mu_{t}=\exp \left(\int_{0}^{t} \frac{1}{4} \operatorname{Tr}\left[\Pi_{u}^{0} \frac{\partial}{\partial u}\left(-J_{0} J_{u}\right)\right] d u\right) .
$$

We deduce from Lemma 3.1 the following local model for the parallel transport in the bundle of holomorphic sections along the path $t \mapsto J_{t}$ for $t \in \mathbb{R}$.

Proposition 3.2. For any $t \in[0,1]$, the following formula holds,

$$
\mathscr{P}_{t}\left(\frac{\partial}{\partial t} \mathscr{P}_{t} \mathscr{P}_{0}\right)=-\frac{1}{4} \operatorname{Tr}\left[\Pi_{t}^{0} \frac{\partial}{\partial t}\left(-J_{0} J_{t}\right)\right] \mathscr{P}_{t} \mathscr{P}_{0}
$$

In particular, we have

$$
\mathscr{P}_{t} \frac{\partial}{\partial t}\left(\mu_{t} \mathscr{P}_{t} \mathscr{P}_{0}\right)=0
$$

Furthermore, the following equality holds,

$$
\mathscr{P}_{t} \mathscr{P}_{0}(0,0)=\left|\mu_{t}\right|^{-2} \text {. }
$$

Proof. First note that for any $Z, Z^{\prime} \in \mathbb{R}^{2 n}$, we have

$$
\begin{aligned}
\frac{\partial}{\partial t} \mathscr{P}_{t}\left(Z, Z^{\prime}\right) & =-\frac{\pi}{2}\left\langle\frac{\partial}{\partial t}\left(-J_{0} J_{t}\right)\left(Z-Z^{\prime}\right),\left(Z-Z^{\prime}\right)\right\rangle \mathscr{P}_{t}\left(Z, Z^{\prime}\right) \\
& =-\frac{\pi}{2}\left\langle\left(-J_{t} \frac{\partial}{\partial t} J_{t}\right)\left(Z-Z^{\prime}\right),\left(Z-Z^{\prime}\right)\right\rangle_{t} \mathscr{P}_{t}\left(Z, Z^{\prime}\right) .
\end{aligned}
$$

Differentiating the identity $J_{t}^{2}=-\mathrm{Id}$ with respect to $t \in \mathbb{R}$, we get the formulas $P_{t}^{(0,1)}\left(\frac{\partial}{\partial t} J_{t}\right)=\left(\frac{\partial}{\partial t} J_{t}\right) P_{t}^{(1,0)}$ and $\operatorname{Tr}\left[-J_{t} \frac{\partial}{\partial t} J_{t}\right]=0$. On the other hand, in (3.6)-(3.9) and 
in Lemma 3.1, we are free to replace 0 by $u$ for any $u \in \mathbb{R}$. Setting $t=u$, we get $A_{t}^{t}=\operatorname{Id}_{\mathbb{R}^{2 n}}, \overline{\Pi_{t}^{t}}=P_{t}^{(0,1)}, \mathscr{P}_{t} \mathscr{P}_{u}=\mathscr{P}_{t}$, so that using $(3.13)$, we get

$$
\begin{aligned}
& \int_{\mathbb{R}^{2 n}} \mathscr{P}_{t}(Z, \widetilde{Z}) \frac{\partial}{\partial t} \mathscr{P}_{t}\left(\widetilde{Z}, Z^{\prime}\right) d \widetilde{Z} \\
& =-\frac{\pi}{2}\left\langle\left(-J_{t} \frac{\partial}{\partial t} J_{t}\right) P_{t}^{(1,0)}\left(Z-Z^{\prime}\right), P_{t}^{(1,0)}\left(Z-Z^{\prime}\right)\right\rangle_{t} \mathscr{P}_{t}\left(Z, Z^{\prime}\right) \\
& -\frac{1}{4} \operatorname{Tr}\left[-J_{t} \frac{\partial}{\partial t} J_{t}\right] \mathscr{P}_{t}\left(Z, Z^{\prime}\right) \\
& =-\frac{\pi}{2}\left\langle\left(\frac{\partial}{\partial t}\left(-J_{0} J_{t}\right)\right) P_{t}^{(1,0)}\left(Z-Z^{\prime}\right),\left(Z-Z^{\prime}\right)\right\rangle \mathscr{P}_{t}\left(Z, Z^{\prime}\right) .
\end{aligned}
$$

This can also be deduced by the analogous computations in $[43, \S 2]$. We then deduce (3.19) from (3.13) and (3.23), using the fact from (3.9) that $P_{t}^{(1,0)} \overline{\Pi_{t}^{0}}=0$ and the fact from (3.7) that $A_{t}^{0} J_{0}=J_{t} A_{t}^{0}$, so that (3.8) implies $\Pi_{t}^{0}=P_{t}^{(0,1)} A_{t}^{0}$. Then (3.20) is a straightforward consequence of (3.18) and (3.19) using $\mathscr{P}_{t} \mathscr{P}_{t}=\mathscr{P}_{t}$. Finally, recall from (3.8) that $A_{0}^{t}=\Pi_{0}^{t}+\overline{\Pi_{0}^{t}}$, and note that

$$
\frac{\partial}{\partial t} \operatorname{det}\left(A_{t}^{0}\right)^{\frac{1}{2}}=-\frac{1}{4} \operatorname{Tr}\left[A_{t}^{0} \frac{\partial}{\partial t}\left(-J_{0} J_{t}\right)\right] \operatorname{det}\left(A_{t}^{0}\right)^{\frac{1}{2}} .
$$

Then as $A_{0}^{0}=\mathrm{Id}$, formula (3.21) follows from (3.11) and (3.18) by integrating (3.24).

We end this section with a study of the holomorphic properties of our local model. Let $z=\left(z_{1}, \ldots, z_{n}\right) \in \mathbb{C}^{n}$ denote the complex coordinates of $\mathbb{C}^{n} \simeq \mathbb{R}^{2 n}$, defined by $z_{j}=Z_{2 j}+\sqrt{-1} Z_{2 j+1}$ for all $1 \leqslant j \leqslant n$. Then by the results of [43, $\left.\S 2\right]$, or by explicit computations from (3.3), we have the following.

Lemma 3.3. For any $F(Z) \in \mathbb{C}[Z]$, there is $\widetilde{\mathcal{Q}}(F)\left(z, \bar{z}^{\prime}\right) \in \mathbb{C}\left[z, \bar{z}^{\prime}\right]$ in the complex coordinates above such that

$$
\int_{\mathbb{R}^{2 n}} \mathscr{P}_{0}(Z, \widetilde{Z}) F(\widetilde{Z}) \mathscr{P}_{0}\left(\widetilde{Z}, Z^{\prime}\right) d \widetilde{Z}=\widetilde{\mathcal{Q}}(F)\left(z, \bar{z}^{\prime}\right) \mathscr{P}_{0}\left(Z, Z^{\prime}\right)
$$

An important remark at this point is that the statement of Lemma 3.3 does not depend on the choice of real coordinates $Z, Z^{\prime} \in \mathbb{R}^{2 n}$, so that Lemma 3.3 holds replacing $J_{0}$ by $J_{t}$ in all that precedes, for any $t \in \mathbb{R}$. In fact, the complex coordinates of $\left(\mathbb{R}^{2 n}, J_{0}\right)$ described above correspond to the basis of $V_{0}^{(1,0)} \subset \mathbb{C}^{2 n}$ given by

$$
\frac{\partial}{\partial z_{j}}=P_{0}^{(1,0)} \frac{\partial}{\partial Z_{2 j}}, \quad \text { for all } \quad 1 \leqslant j \leqslant n .
$$

For any $t \in \mathbb{R}$, recall (3.10) and set

$$
\frac{\partial}{\partial z_{t, j}}=\Pi_{t}^{0} \frac{\partial}{\partial Z_{2 j}}, \quad \text { for all } \quad 1 \leqslant j \leqslant n .
$$


Let $z_{t}=\left(z_{t, 1}, \ldots, z_{t, n}\right)$ be the associated complex coordinates of $\left(\mathbb{R}^{2 n}, J_{t}\right)$. Then using an appropriate change of basis, we have the following straightforward generalization of Lemma 3.3.

Lemma 3.4. For any $F(Z) \in \mathbb{C}[Z]$ and for any $t \in \mathbb{R}$, there is $\widetilde{\mathcal{Q}}_{t}(F)\left(z_{t}, \bar{z}_{t}^{\prime}\right) \in \mathbb{C}\left[z_{t}, \bar{z}_{t}^{\prime}\right]$ in the complex coordinates of $\left(\mathbb{R}^{2 n}, J_{t}\right)$ defined above such that

$$
\int_{\mathbb{R}^{2 n}} \mathscr{P}_{t}(Z, \widetilde{Z}) F(\widetilde{Z}) \mathscr{P}_{t}\left(\widetilde{Z}, Z^{\prime}\right) d \widetilde{Z}=\widetilde{\mathcal{Q}}_{t}(F)\left(z_{t}, \bar{z}_{t}^{\prime}\right) \mathscr{P}_{0}\left(Z, Z^{\prime}\right)
$$

By the description of $\Pi_{t}^{0}$ as the projection operator on $V_{t}^{(1,0)}$ with kernel $V_{0}^{(0,1)}$, we see that (3.27) defines a basis of $V_{t}^{(1,0)}$, so that

$$
\left\{\frac{\partial}{\partial z_{t, j}}, \frac{\partial}{\partial \bar{z}_{j}}\right\}_{j=1}^{n}
$$

defines a basis of $\mathbb{C}^{n}=V_{t}^{(1,0)} \oplus V_{0}^{(0,1)}$ as in (3.10).

For any $t \in \mathbb{R}$ and $F \in \mathbb{C}\left[Z, Z^{\prime}\right]$, write $F \mathscr{P}_{t}$ the operator on $L^{2}\left(\mathbb{R}^{2 n}\right)$ whose Schwartz kernel with respect to $d Z$ is given by $F \mathscr{P}_{t}\left(Z, Z^{\prime}\right)=F\left(Z, Z^{\prime}\right) \mathscr{P}_{t}\left(Z, Z^{\prime}\right)$. In the same way, we write $F \mathscr{P}_{t} \mathscr{P}_{0}$ for the operator with Schwartz kernel $F\left(Z, Z^{\prime}\right) \mathscr{P}_{t} \mathscr{P}_{0}\left(Z, Z^{\prime}\right)$. We can now state the following fundamental property of our local model.

Proposition 3.5. For any $F\left(Z, Z^{\prime}\right) \in \mathbb{C}\left[Z, Z^{\prime}\right]$, there exists $Q_{t}(F)\left(z_{t}, \bar{z}^{\prime}\right) \in \mathbb{C}\left[z_{t}, \bar{z}^{\prime}\right]$ of the same parity as $F$ for any $t \in \mathbb{R}$, such that

$$
\mathscr{P}_{t}\left(F \mathscr{P}_{t} \mathscr{P}_{0}\right) \mathscr{P}_{0}=Q_{t}(F) \mathscr{P}_{t} \mathscr{P}_{0}
$$

Proof. The existence of a polynomial $Q_{t}(F)$ in $Z, Z^{\prime} \in \mathbb{R}^{2 n}$ of the same parity as $F$ satisfying (3.30) follows from two applications of (3.12) and the fact that $\mathscr{P}_{t} \mathscr{P}_{t}=\mathscr{P}_{t}$. This together with the definition (3.30) of $Q_{t}(F)$ gives

$$
\mathscr{P}_{t}\left(Q_{t}(F) \mathscr{P}_{t} \mathscr{P}_{0}\right)=\mathscr{P}_{t}\left(F \mathscr{P}_{t} \mathscr{P}_{0}\right) \mathscr{P}_{0}=Q_{t}(F) \mathscr{P}_{t} \mathscr{P}_{0}
$$

Thus considering $Q_{t}(F)\left(Z, Z^{\prime}\right)$ as a polynomial in $Z \in \mathbb{R}^{2 n}$, we get from Lemma 3.4 that $Q_{t}(F)$ depends only on $z_{t}$ and $Z^{\prime}$. Using

$$
\left(Q_{t}(F) \mathscr{P}_{t} \mathscr{P}_{0}\right) \mathscr{P}_{0}=\mathscr{P}_{t}\left(F \mathscr{P}_{t} \mathscr{P}_{0}\right) \mathscr{P}_{0}=Q_{t}(F) \mathscr{P}_{t} \mathscr{P}_{0}
$$

we deduce in the same way that $Q_{t}(F)$ depends only on $z_{t}$ and $\bar{z}^{\prime}$, from which we deduce Proposition 3.5.

\subsection{Criterion for Toeplitz operators}

Consider the setting and notations of Section 2.3, and let $p_{0} \in \mathbb{N}$ as in Theorem 2.2 for $U=B$ be fixed. Recall that we identified $E_{p}$ over $[0,1] \times X$ with $E_{p, t}=E_{t} \otimes L^{p}$ over $X$ for any $t \in[0,1]$, where $\left(L, h^{L}, \nabla^{L}\right)$ does not depend on $t$. Then for any $t \in[0,1]$ and 
$g_{t} \in \mathscr{C}^{\infty}\left(X, E_{t} \otimes E_{0}^{*}\right)$, we can define the Berezin-Toeplitz quantization of $g_{t}$ as a family indexed by $p \in \mathbb{N}^{*}$ of linear maps from $\mathscr{H}_{p, 0}$ to $\mathscr{H}_{p, t}$ by the formula

$$
P_{p, t} g_{t} P_{p, 0}: \mathscr{C}^{\infty}\left(X, E_{p, 0}\right) \rightarrow \mathscr{C}^{\infty}\left(X, E_{p, t}\right)
$$

As $\operatorname{dim} \mathscr{H}_{p, t}<\infty$ for all $p \in \mathbb{N}^{*}$ and $t \in \mathbb{R}$, this operator admits a smooth Schwartz kernel in $\mathscr{C}^{\infty}\left(X \times X, E_{p, t}^{*} \otimes E_{p, 0}\right)$ with respect to $d v_{X}$. Recalling Notation 2.8, we then have the following result.

Lemma 3.6. There is a family $\left\{\mathcal{Q}_{r, t, x_{0}}(g)\left(Z, Z^{\prime}\right) \in E_{t, x_{0}} \otimes E_{0, x_{0}}^{*}\right\}_{r \in \mathbb{N}}$ of polynomials in $Z, Z^{\prime} \in \mathbb{R}^{2 n}$ of the same parity as $r$, smooth in $x_{0} \in X$ and $t \in[0,1]$, such that the following asymptotic expansion holds,

$$
p^{-n}\left(P_{p, t} g_{t} P_{p, 0}\right)_{x_{0}}\left(Z, Z^{\prime}\right) \cong \sum_{r=0}^{\infty} \mathcal{Q}_{r, t, x_{0}}(g) \mathscr{P}_{t, x_{0}} \mathscr{P}_{0}\left(\sqrt{p} Z, \sqrt{p} Z^{\prime}\right) p^{-\frac{r}{2}}+\mathscr{O}\left(p^{-\infty}\right) .
$$

Furthermore, for all $x_{0} \in X, t \in[0,1], Z, Z^{\prime} \in \mathbb{R}^{2 n}$, we have

$$
\mathcal{Q}_{0, t, x_{0}}(g)\left(Z, Z^{\prime}\right)=g_{t}\left(x_{0}\right) \text {. }
$$

Proof. Using the results of Section 3.1, and in particular Lemma 3.1, the proof of (3.34) and (3.35) is a straightforward adaptation of the proof of Lemma 2.9 in [35, Lem.3.3], using the asymptotic expansion (2.23) of the Bergman kernel.

For any $t \in \mathbb{R}$ and $p \in \mathbb{N}^{*}$, let $\|\cdot\|_{p, 0, t}$ be the operator norm induced by $\|\cdot\|_{p, 0}$ and $\|\cdot\|_{p, t}$ on the space $L\left(\mathscr{H}_{p, 0}, \mathscr{H}_{p, t}\right)$ of bounded operators from $\mathscr{H}_{p, 0}$ to $\mathscr{H}_{p, t}$. The following fundamental result is a converse to Lemma 3.6, and gives a criterion for a sequence of operators in $L\left(\mathscr{H}_{p, 0}, \mathscr{H}_{p, t}\right)$ for all $p \in \mathbb{N}^{*}$ to behave like a Toeplitz operator, that is to admit an asymptotic expansion as $p \rightarrow+\infty$ in terms of Berezin-Toeplitz operators (3.33).

Theorem 3.7. Let $\left\{T_{p, t} \in L\left(\mathscr{H}_{p, 0}, \mathscr{H}_{p, t}\right)\right\}_{p \in \mathbb{N}^{*}}$ be a family of bounded operators from $\mathscr{H}_{p, 0}$ to $\mathscr{H}_{p, t}$, smooth in $t \in[0,1]$, and assume that for any $p \in \mathbb{N}^{*}$ and $t \in \mathbb{R}$, the induced operator $T_{p, t}=P_{p, t} T_{p, t} P_{p, 0}: \mathscr{C}^{\infty}\left(X, E_{p, 0}\right) \rightarrow \mathscr{C}^{\infty}\left(X, E_{p, t}\right)$ satisfies

$$
p^{-n} T_{p, t}\left(Z, Z^{\prime}\right) \cong \sum_{r=0}^{\infty} Q_{r, t, x_{0}} \mathscr{P}_{t, x_{0}} \mathscr{P}_{0}\left(\sqrt{p} Z, \sqrt{p} Z^{\prime}\right) p^{-\frac{r}{2}}+\mathscr{O}\left(p^{-\infty}\right),
$$

for a family $\left\{\mathcal{Q}_{r, t, x_{0}}\left(Z, Z^{\prime}\right) \in E_{t, x_{0}} \otimes E_{0, x_{0}}^{*}\right\}_{r \in \mathbb{N}}$ of polynomials in $Z, Z^{\prime} \in \mathbb{R}^{2 n}$ of the same parity as $r$, smooth in $x_{0} \in X$ and $t \in[0,1]$.

Then there exist a family $\left\{g_{l, t} \in \mathscr{C}^{\infty}\left(X, E_{t} \otimes E_{0}^{*}\right)\right\}_{l \in \mathbb{N}}$, smooth in $t \in[0,1]$, such that for all $k \geqslant 0$, there exists $C_{k}>0$ such that

$$
\left\|T_{p, t}-\sum_{l=0}^{k-1} p^{-l} P_{p, t} g_{l, t} P_{p, 0}\right\|_{p, 0, t} \leqslant C_{k} p^{-k}
$$

for all $p \in \mathbb{N}^{*}$ and $t \in[0,1]$. 
The proof of Theorem 3.7 is parallel to the proof of the analogous results in [43, $\S 4.2],[35, \S 4]$, and will occupy the rest of this section. The main additional difficulty is that we are working with two sets $z, z_{t}$ of complex coordinates of $\mathbb{R}^{2 n}$ for two different complex structures $J_{0}$ and $J_{t}$ as in Section 3.1. As it will appear in the proof of Lemma 3.12, this is solved using the fact that the spaces $V_{0}^{(1,0)}$ and $V_{t}^{(0,1)}$ in $(3.10)$ are transverse in $\mathbb{C}^{2 n}$. Another difference is that we can't assume $T_{p, t}$ to be self-adjoint in this context.

Following $[43, \S 4.2]$, we will construct inductively the sequence $\left\{g_{l, t} \in \mathscr{C}^{\infty}\left(X, E_{t} \otimes\right.\right.$ $\left.\left.E_{0}^{*}\right)\right\}_{l \in \mathbb{N}}$ for any $t \in[0,1]$ such that $(3.37)$ holds. Let us start with the case $k=0$ in (3.37). For any $t \in[0,1]$ and $x_{0} \in X$, we set

$$
g_{0, t}\left(x_{0}\right)=\mathcal{Q}_{0, t, x_{0}}(0,0) \in E_{t, x_{0}} \otimes E_{0, x_{0}}^{*} .
$$

Then $g_{0, t}\left(x_{0}\right)$ is smooth in $t \in[0,1]$. We will show that

$$
T_{p, t}=P_{p, t} g_{0, t} P_{p, 0}+O\left(p^{-1}\right) .
$$

The proof of (3.39) is the result of Proposition 3.8 and Proposition 3.14. In the proof of these propositions, we fix $t \in[0,1]$.

Proposition 3.8. In the conditions of Theorem 3.7, we have

$$
\mathcal{Q}_{0, t, x_{0}}\left(Z, Z^{\prime}\right)=\mathcal{Q}_{0, t, x_{0}}(0,0) \in E_{t, x_{0}} \otimes E_{0, x_{0}}^{*}
$$

for all $t \in[0,1], x_{0} \in X$ and $Z, Z^{\prime} \in \mathbb{R}^{2 n}$.

Proof. The proof is divided in the series of Lemmas Lemma 3.9 - Lemma 3.13. Recall from Section 3.1 that $z^{\prime}$ denotes the holomorphic coordinate in $Z^{\prime} \in \mathbb{R}^{2 n}$ associated to $J_{0, x_{0}}$ as in (3.26) and that $z_{t}$ denotes the holomorphic coordinate in $Z \in \mathbb{R}^{2 n}$ associated to $J_{t, x_{0}}$ as in (3.27). Our first observation is as follows.

Lemma 3.9. $\mathcal{Q}_{0, t, x_{0}}$ only depends on $z_{t}, \bar{z}^{\prime}$, so that there is $\mathcal{Q}_{t, x_{0}}\left(z_{t}, \bar{z}^{\prime}\right) \in E_{t, x_{0}} \otimes E_{0, x_{0}}^{*}$, polynomial in $z_{t}, \bar{z}^{\prime}$, such that for all $Z, Z^{\prime} \in \mathbb{R}^{2 n}$,

$$
\mathcal{Q}_{0, t, x_{0}}\left(z, \bar{z}^{\prime}\right)=\mathcal{Q}_{0, t, x_{0}}\left(Z, Z^{\prime}\right) .
$$

Proof. By (3.36), we know that

$$
p^{-n} T_{p, t, x_{0}}\left(Z, Z^{\prime}\right) \cong \mathcal{Q}_{r, t, x_{0}} \mathscr{P}_{t, x_{0}} \mathscr{P}_{x_{0}}\left(\sqrt{p} Z, \sqrt{p} Z^{\prime}\right)+\mathscr{O}\left(p^{-\frac{1}{2}}\right) .
$$

By Proposition 2.7 in the context of Section 2.3, we thus get in the notations of Section 3.1,

$$
p^{-n}\left(P_{p, t} T_{p, t} P_{p, 0}\right)_{x_{0}}\left(Z, Z^{\prime}\right) \cong \mathscr{P}_{t, x_{0}}\left(\mathcal{Q}_{0, t, x_{0}} \mathscr{P}_{t, x_{0}} \mathscr{P}_{0}\right) \mathscr{P}_{0}\left(\sqrt{p} Z, \sqrt{p} Z^{\prime}\right)+\mathscr{O}\left(p^{-\frac{1}{2}}\right) .
$$

On the other hand, from (3.42), (3.43) and the formula $P_{p, t} T_{p, t}=T_{p, t}$, we deduce

$$
\mathcal{Q}_{0, t, x_{0}} \mathscr{P}_{t, x_{0}} \mathscr{P}_{0, x_{0}}=\mathscr{P}_{t, x_{0}}\left(\mathcal{Q}_{0, t, x_{0}} \mathscr{P}_{t, x_{0}} \mathscr{P}_{0}\right) \mathscr{P}_{0}
$$

From Proposition 3.5, this implies that $\mathcal{Q}_{0, t, x_{0}}$ only depends on $z_{t}, \bar{z}^{\prime}$. 
Recall from Section 2.3 that $\tau_{t}^{E} \in E_{t} \otimes E_{0}^{*}$ is induced by parallel transport with respect to $\nabla^{E}$ along horizontal lines of $[0,1] \times X$ going from 0 to $t \in[0,1]$. For all $Z, Z^{\prime} \in \mathbb{R}^{2 n}$, let us write

$$
\mathcal{Q}_{x_{0}}\left(z_{t}, \bar{z}^{\prime}\right)=\tau_{t, x_{0}}^{E,-1} \mathcal{Q}_{0, t, x_{0}}\left(z_{t}, \bar{z}^{\prime}\right) \in \operatorname{End}\left(E_{0, x_{0}}\right)
$$

so that 3.9 implies $\mathcal{Q}_{x_{0}}\left(Z, Z^{\prime}\right)=\mathcal{Q}_{x_{0}}\left(z_{t}, \bar{z}^{\prime}\right)$. For any $x_{0} \in X$, let $\mathcal{Q}_{x_{0}}=\sum_{i \geqslant 0} \mathcal{Q}_{x_{0}}^{(i)}$ be the decomposition of $\mathcal{Q}_{x_{0}}$ in homogeneous polynomials $\mathcal{Q}_{x_{0}}^{(i)}$ of degree $i$. We will show that $\mathcal{Q}_{x_{0}}^{(i)}$ vanishes identically for $i>0$, that is

$$
\mathcal{Q}_{x_{0}}^{(i)}\left(z_{t}, \bar{z}^{\prime}\right)=0 \text { for all } x_{0} \in X, i>0 .
$$

The first step is to prove

$$
\mathcal{Q}_{x_{0}}^{(i)}\left(z_{t}, 0\right)=0 \text { for all } x_{0} \in X, i>0 .
$$

Recall the smooth family of vertical charts $\psi$ in Section 2.3. For $x \in X, Z^{\prime} \in \mathbb{R}^{2 n} \simeq T_{x} X$ and $y=\psi_{x}\left(Z^{\prime}\right)$, set

$$
\begin{aligned}
& F^{(i)}(x, y)=\mathcal{Q}_{x}^{(i)}\left(0, \bar{z}^{\prime}\right) \in \operatorname{End}\left(E_{0, x}\right), \\
& \widetilde{F}^{(i)}(x, y)=\left(F^{(i)}(y, x)\right)^{*} \in \operatorname{End}\left(E_{0, y}\right) .
\end{aligned}
$$

Then $F^{i}$ and $\widetilde{F}^{(i)}$ define smooth sections on a neighborhood of the diagonal of $X \times X$. Clearly, the $\widetilde{F}^{(i)}(x, y)$ 's need not be polynomials in $z_{t}$ and $\bar{z}^{\prime}$.

Denote by $d(\cdot, \cdot)$ the Riemannian distance on $\left(X, g_{0}^{T X}\right)$. Since we wish to define global operators induced by these kernels, we use a cut-off function in the neighborhood of the diagonal. Pick a smooth function $\eta \in \mathscr{C}^{\infty}(\mathbb{R})$ such that $\eta(u)=1$ for $|u| \leqslant \varepsilon_{0} / 2$ and $\eta(u)=0$ for $|u| \geqslant \varepsilon_{0}$. We denote by $P_{p, t} P_{p, 0} F^{(i)}$ and $\widetilde{F}^{(i)} P_{p, 0} P_{p, t}$ the operators defined by the kernels

$$
\eta(d(x, y))\left(P_{p, t} \tau_{t}^{E} P_{p, 0}\right)(x, y) F^{(i)}(x, y) \text { and } \eta(d(x, y)) \widetilde{F}^{(i)}(x, y)\left(P_{p, 0} \tau_{t}^{E,-1} P_{p, t}\right)(x, y)
$$

with respect to $d v_{X}(y)$. Set

$$
\mathscr{T}_{p}=T_{p, t}-\sum_{i \geqslant 0}\left(P_{p, t} P_{p, 0} F^{(i)}\right) p^{\frac{i}{2}}
$$

From (3.34), (3.36) and (3.50), we deduce that in the sense of (2.26) for $Z=0$ and any $x_{0} \in X$, we have

$$
p^{-n} \mathscr{T}_{p, x_{0}}\left(0, Z^{\prime}\right) \cong \sum_{r=1}^{\infty}\left(R_{r} \mathscr{P}_{t, x_{0}} \mathscr{P}_{0}\right)\left(0, \sqrt{p} Z^{\prime}\right) p^{-\frac{r}{2}}+\mathscr{O}\left(p^{-\infty}\right),
$$

for some polynomials $R_{r, x_{0}}$ of the same parity as $r$. 
Lemma 3.10. There exists $C>0$ such that for any $p \in \mathbb{N}^{*}$ and $s \in \mathscr{C}^{\infty}\left(X, E_{p, 0}\right)$, we have

$$
\begin{aligned}
\left\|\mathscr{T}_{p} s\right\|_{p, t} & \leqslant C p^{-\frac{1}{2}}\|s\|_{p, 0}, \\
\left\|\mathscr{T}_{p}^{*} s\right\|_{p, t} & \leqslant C p^{-\frac{1}{2}}\|s\|_{p, 0} .
\end{aligned}
$$

Proof. This is a consequence of the vanishing of the term of order 0 in (3.51). The proof is the same than the proof of the analogous result in [43, Lem.4.13].

For any $x_{0} \in X$ and $Z, Z^{\prime} \in \mathbb{R}^{2 n} \simeq T_{x_{0}} X$ such that $|Z|,\left|Z^{\prime}\right|<\varepsilon_{0}$, recall that $\widetilde{F}_{x_{0}}^{(i)}\left(Z, Z^{\prime}\right) \in \operatorname{End}\left(E_{0, x_{0}}\right)$ denotes the image of $\widetilde{F}^{(i)}(x, y) \in \operatorname{End}\left(E_{0, x_{0}}\right)$, with $x=\psi_{x_{0}}(Z)$, $y=\psi_{x_{0}}\left(Z^{\prime}\right)$ in the trivialization around $x_{0} \in X$ defined in Section 2.3. Let us consider the following Taylor expansion, for any $k \in \mathbb{N}$,

$$
\widetilde{F}_{x_{0}}^{(i)}\left(0, Z^{\prime}\right)=\sum_{|\alpha| \leqslant k} \frac{\partial^{|\alpha|} \widetilde{F}_{x_{0}}^{(i)}}{\partial Z^{\prime \alpha}}(0,0) \frac{\left(\sqrt{p} Z^{\prime}\right)^{\alpha}}{\alpha !} p^{-\frac{|\alpha|}{2}}+O\left(\left|Z^{\prime}\right|^{k+1}\right) .
$$

The next step of the proof of Proposition 3.8 is the following.

Lemma 3.11. For any $x_{0} \in X$, we have

$$
\frac{\partial^{|\alpha|} \widetilde{F}_{x_{0}}^{(i)}}{\partial Z^{\prime \alpha}}(0,0)=0 \text { for } i-|\alpha|>0
$$

Proof. The definition (3.50) of $\mathscr{T}_{p}$ shows that

$$
\mathscr{T}_{p}^{*}=T_{p, t}^{*}-\sum_{i \geqslant 0} p^{\frac{i}{2}}\left(\widetilde{F}^{(i)} P_{p, 0} P_{p, t}\right)
$$

Pick $x_{0} \in X$, and let us develop the sum on the right-hand side. Combining the Taylor expansion (3.53) with the expansion (3.34) where the roles of $J_{0}$ and $J_{t}$ are swapped, for any $k \geqslant \operatorname{deg} \mathcal{Q}_{x_{0}}+1$ we obtain

$$
\begin{array}{r}
p^{-n} \sum_{i \geqslant 0}\left(\widetilde{F}^{(i)} P_{p, 0} P_{p, t}\right)_{x_{0}}\left(0, Z^{\prime}\right) p^{\frac{i}{2}} \cong \sum_{i \geqslant 0} \sum_{|\alpha|, r \leqslant k} \mathcal{Q}_{r, t, x_{0}}\left(\tau^{E,-1}\right) \mathscr{P}_{0} \mathscr{P}_{t, x_{0}}\left(0, \sqrt{p} Z^{\prime}\right) \\
\frac{\partial^{|\alpha|} \widetilde{F}_{x_{0}}^{(i)}}{\partial Z^{\prime \alpha}}(0,0) \frac{\left(\sqrt{p} Z^{\prime}\right)^{\alpha}}{\alpha !} p^{\frac{i-|\alpha|-r}{2}}+\mathscr{O}\left(p^{\frac{\operatorname{deg} \mathcal{Q}_{x_{0}-k-1}}{2}}\right) .
\end{array}
$$

Having in mind the second inequality of (3.52), this is only possible if for every $j>0$, the coefficients of $p^{\frac{j}{2}}$ in the right-hand side of (3.56) vanish. Thus, we have for any $j>0$,

$$
\sum_{i=j}^{\operatorname{deg} \mathcal{Q}_{x_{0}}} \sum_{j+r=i-|\alpha|} \mathcal{Q}_{r, t, x_{0}}\left(\tau^{E,-1}\right)\left(0, \sqrt{p} Z^{\prime}\right) \frac{\partial^{|\alpha|} \widetilde{F}_{x_{0}}^{(i)}}{\partial Z^{\prime \alpha}}(0,0) \frac{\left(\sqrt{p} Z^{\prime}\right)^{\alpha}}{\alpha !}=0
$$


Note that (3.48) implies that $\widetilde{F}^{(i)}=0$ for $i>\operatorname{deg} \mathcal{Q}_{x_{0}}$, so that (3.54) automatically holds in that case. From (3.57), we will prove by a descending recurrence on $j>0$ that (3.54) holds for $i-|\alpha|>j$. As the first step of the recurrence, let us take $j=\operatorname{deg} \mathcal{Q}_{x_{0}}$ in (3.57). Since $\mathcal{Q}_{0, t, x_{0}}\left(\tau^{E,-1}\right)=\tau_{t, x_{0}}^{E,-1}$ is invertible, we get immediately $\widetilde{F}_{x_{0}}^{(j)}(0,0)=0$ in that case. Hence (3.54) holds for $i-|\alpha| \geqslant \operatorname{deg} \mathcal{Q}_{x_{0}}$. Assume that (3.54) holds for $i-|\alpha|>j_{0}>0$. Then for $j=j_{0}$, the coefficient with $r>0$ in (3.57) is zero. By the invertibility of $\mathcal{Q}_{0, t, x_{0}}\left(\tau^{E,-1}\right)=\tau_{t, x_{0}}^{E,-1}$ once again, (3.57) reads

$$
\sum_{\alpha \in \mathbb{N}^{2 n}} \frac{\partial^{|\alpha|} \widetilde{F}_{x_{0}}^{\left(j_{0}+|\alpha|\right)}}{\partial Z^{\prime \alpha}}\left(x_{0}, 0\right) \frac{\left(\sqrt{p} Z^{\prime}\right)^{\alpha}}{\alpha !}=0,
$$

which entails (3.54) for $i-|\alpha| \geqslant j_{0}$. The proof of (3.54) is complete.

Lemma 3.12. For any $x_{0} \in X$, we have

$$
\frac{\partial^{|\alpha|} \mathcal{Q}_{x_{0}}^{(i)}}{\partial z_{t}^{\alpha}}(0,0)=0, \text { for all }|\alpha| \leqslant i .
$$

Therefore $\mathcal{Q}_{x_{0}}^{(i)}\left(z_{t}, 0\right)=0$ for all $i>0$ and $Z \in \mathbb{R}^{2 n}$, so that (3.47) holds true. Moreover,

$$
\mathcal{Q}_{x_{0}}^{(i)}\left(0, \bar{z}^{\prime}\right)=0 \text { for all } x_{0} \in X, i>0 \text { and all } Z \in \mathbb{R}^{2 n} .
$$

Proof. Let us start with some preliminary observations. From Lemma 3.11 and (3.56), we get for any $x_{0} \in X$,

$$
\begin{aligned}
& p^{-n} \sum_{i \geqslant 0}\left(\widetilde{F}^{(i)} P_{p, 0} P_{p, t}\right)_{x_{0}}\left(0, Z^{\prime}\right) p^{\frac{i}{2}} \\
& \cong \sum_{|\alpha|=i} \frac{\partial^{|\alpha|} \widetilde{F}_{x_{0}}^{(i)}}{\partial Z^{\prime \alpha}}(0,0) \frac{\left(\sqrt{p} Z^{\prime}\right)^{\alpha}}{\alpha !} \tau_{t, x_{0}}^{E,-1} \mathscr{P}_{0} \mathscr{P}_{t, x_{0}}\left(0, \sqrt{p} Z^{\prime}\right)+\mathscr{O}\left(p^{-\frac{1}{2}}\right) .
\end{aligned}
$$

On the other hand, taking the adjoint of (3.36) and using (3.41), (3.45) we get

$$
p^{-n} T_{p, x_{0}}^{*}\left(0, Z^{\prime}\right) \cong\left(\mathcal{Q}_{x_{0}}\left(\sqrt{p} z_{t}^{\prime}, 0\right)\right)^{*} \tau_{t, x_{0}}^{E,-1} \mathscr{P}_{0} \mathscr{P}_{t, x_{0}}\left(0, \sqrt{p} Z^{\prime}\right)+\mathscr{O}\left(p^{-\frac{1}{2}}\right) .
$$

In view of Lemma 3.10, comparing (3.55) with (3.61) and (3.62) for any $x_{0} \in X$ gives

$$
\widetilde{F}_{x_{0}}^{(i)}\left(0, Z^{\prime}\right)=\left(\mathcal{Q}_{x_{0}}^{(i)}\left(z_{t}^{\prime}, 0\right)\right)^{*}+O\left(\left|Z^{\prime}\right|^{i+1}\right) .
$$

By definition (3.48) of $\widetilde{F}^{(i)}$, we take the adjoint of (3.63) and get

$$
F_{x_{0}}^{(i)}(Z, 0)=\mathcal{Q}_{x_{0}}^{(i)}\left(z_{t}, 0\right)+O\left(|Z|^{i+1}\right) .
$$

Thus in order to prove the Lemma it suffices to show that

$$
\frac{\partial^{|\alpha|}}{\partial z^{\alpha}} F_{x_{0}}^{(i)}(0,0)=0, \text { for all } x_{0} \in X \text { and }|\alpha| \leqslant i .
$$


We prove this by induction over $|\alpha|$. The case $|\alpha|=0$ immediately follows from the fact that $\mathcal{Q}_{x_{0}}^{(i)}\left(z_{t}, \bar{z}^{\prime}\right)$ is a homogeneous polynomial of degree $i>0$. For the induction step, assume that (3.65) holds for $|\alpha|=i_{0}<i$ at all $x_{0} \in X$. Together with (3.64), this implies in particular that all derivatives in $x$ up to order $i_{0}$ of $F^{(i)}(x, y)$ vanish for $x=y$. On the other hand, we know by definition (3.48) of $F^{(i)}$ that

$$
\frac{\partial}{\partial z_{j}^{\prime}} F_{x_{0}}^{(i)}\left(0, Z^{\prime}\right)=0, \text { for all } x_{0} \in X \text { and }\left|Z^{\prime}\right|<\varepsilon_{0} \text {. }
$$

Let us write $j_{\Delta}: \mathbb{R}^{2 n} \rightarrow \mathbb{R}^{2 n} \times \mathbb{R}^{2 n}$ for the diagonal injection. Using the fact that $\widetilde{F}^{(i)}\left(\psi_{x}(Z), \psi_{x}\left(Z^{\prime}\right)\right)=\widetilde{F}_{x}^{(i)}\left(Z, Z^{\prime}\right)$ for all $|Z|,\left|Z^{\prime}\right|<\varepsilon_{0}$ and $x \in X$ in the coordinate charts $\psi_{x}: T_{x} X \rightarrow X$, we then deduce from the induction hypothesis that for any $1 \leqslant j \leqslant n$ and $x \in X$

$$
\frac{\partial^{|\alpha|+1} F_{x}^{(i)}}{\partial z^{\alpha} \partial z_{j}}(0,0)=\left(\frac{\partial}{\partial z_{j}} j_{\Delta}^{*} \frac{\partial^{|\alpha|} F_{x}^{(i)}}{\partial z^{\alpha}}\right)(0)-\frac{\partial^{|\alpha|}}{\partial z^{\alpha}} \frac{\partial F_{x}^{(i)}}{\partial z_{j}^{\prime}}(0,0)=0 .
$$

This gives (3.65). Now recall from (3.29) that the set of vectors $\left\{\partial / \partial z_{j}, \partial / \partial \bar{z}_{j, t}\right\}_{1 \leqslant j \leqslant n}$ form a basis of $\mathbb{C}^{2 n}$, so that (3.64) and (3.65) implies (3.59), and this is equivalent to (3.47). The argument for (3.60) is analogous, setting $\widetilde{F}^{(i)}(x, y):=\left(\mathcal{Q}^{(i)}\left(z_{t}, 0\right)\right)^{*}$ and $F^{(i)}(x, y):=\left(\widetilde{F}^{(i)}(y, x)\right)^{*}$ in $(3.48)$ and swapping their roles in the rest of the argument. This finishes the proof of Lemma 3.12.

Lemma 3.13. We have $\mathcal{Q}_{x_{0}}^{(i)}\left(z_{t}, \bar{z}^{\prime}\right)=0$ for all $x_{0} \in X$ and $i>0$.

Proof. For any $x_{0} \in X$, let us consider the operator

$$
\frac{1}{\sqrt{p}} P_{p, t}\left(\nabla_{v}^{E_{p}} T_{p, t}\right) P_{p, 0} \text { with } v \in \mathscr{C}^{\infty}\left(X, T X_{\mathbb{C}}\right), v_{x_{0}}=\frac{\partial}{\partial z_{t, j}} .
$$

By (2.26) and (3.45), the operator (3.68) admits an expansion as in (3.36) except for the condition on the parity of the polynomials, with leading term at $x_{0} \in X$ equal to

$$
\tau_{t, x_{0}}^{E,-1}\left(\frac{\partial \mathcal{Q}_{x_{0}}}{\partial z_{t, j}}\right)\left(\sqrt{p} z_{t}, \sqrt{p} \bar{z}^{\prime}\right) \mathscr{P}_{x_{0}}\left(\sqrt{p} Z, \sqrt{p} Z^{\prime}\right)
$$

On the other hand, note that the proofs of Lemmas 3.10 to 3.12 did not use the parity condition on the polynomials in Theorem 3.7, so that Lemma 3.12 holds for the operator (3.68). Following the notations above, we thus get for $i>0$,

$$
\frac{\partial \mathcal{Q}_{x_{0}}^{(i+1)}}{\partial z_{t, j}}\left(0, \bar{z}^{\prime}\right)=\left(\frac{\partial \mathcal{Q}_{x_{0}}}{\partial z_{t, j}}\right)^{(i)}\left(0, \bar{z}^{\prime}\right)=0 .
$$

Now (3.47) tells us that the constant term of $\left(\frac{\partial}{\partial z_{j, t}} \mathcal{Q}_{x_{0}}\right)\left(z_{t}, \bar{z}^{\prime}\right)$ vanishes, so that (3.70) holds as well for $i=0$. Then repeating this reasoning with the adjoint of (3.68), we further get for any $i>0$ and $1 \leqslant j \leqslant n$,

$$
\frac{\partial \mathcal{Q}_{x_{0}}^{(i)}}{\partial \bar{z}_{j}^{\prime}}\left(z_{t}, 0\right)=0
$$


By continuing this process, we show by induction that for all $x_{0} \in X, i>0$ and $\alpha \in \mathbb{N}^{n}$,

$$
\frac{\partial^{|\alpha|} \mathcal{Q}_{x_{0}}^{(i)}}{\partial z_{t}^{\alpha}}\left(0, \bar{z}^{\prime}\right)=\frac{\partial^{|\alpha|} \mathcal{Q}_{x_{0}}^{(i)}}{\partial \bar{z}^{\prime \alpha}}\left(z_{t}, 0\right)=0 .
$$

This proves Lemma 3.13 and (3.46) holds true.

Lemma 3.13 finishes the proof of Proposition 3.8.

Proposition 3.14. We have

$$
T_{p, t}=P_{p, t} g_{0, t} P_{p, 0}+O\left(p^{-1}\right),
$$

i.e., relation (3.39) holds true in the sense of (3.37).

Proof. Comparing the asymptotic expansions (3.36) and (3.34) of both sides of (3.73) up to order 2, as in the proof of the analogous result in [43, Prop.4.17], it suffices to prove that for any $x_{0} \in X$ and $Z, Z^{\prime} \in \mathbb{R}^{2 n}$,

$$
\left(\mathcal{Q}_{1, t, x_{0}}-\mathcal{Q}_{1, t, x_{0}}\left(g_{0}\right)\right)\left(Z, Z^{\prime}\right)=0 .
$$

The left hand side of (3.74) is the polynomial associated with the first coefficient of the expansion as in (3.36) of

$$
\sqrt{p}\left(T_{p, t}-P_{p, t} g_{0, t} P_{p}\right) .
$$

As before, we see that this operator satisfies the hypotheses of Lemmas 3.10 to 3.13, so that all the homogeneous components of degree $i>0$ of $\mathcal{Q}_{1, t, x_{0}}-\mathcal{Q}_{1, t, x_{0}}\left(g_{0}\right)$ vanish. Furthermore, $\mathcal{Q}_{1, t, x_{0}}-Q_{1, t, x_{0}}\left(g_{0}\right)$ is an odd polynomial, so that in particular its constant term vanishes as well. This shows (3.74).

By (3.73), the operator $p\left(T_{p, t}-P_{p, t} g_{0, t} P_{p, 0}\right)$ satisfies the hypotheses of Theorem 3.7, so that Proposition 3.8 and Proposition 3.14 applied to $p\left(T_{p, t}-P_{p, t} g_{0, t} P_{p, 0}\right)$ yield $g_{1, t} \in$ $\mathscr{C}^{\infty}\left(X, E_{t} \otimes E_{0}^{*}\right)$ such that

$$
T_{p, t}=P_{p, t} g_{0, t} P_{p, 0}+P_{p, t} g_{1, t} P_{p, 0}+O\left(p^{-2}\right)
$$

We can then continue this process to get (3.37) for any $q \in \mathbb{N}$ by induction. This completes the proof of Theorem 3.7.

\subsection{Parallel transport as a Toeplitz operator}

In this section, we show that the parallel transport in the quantum bundle of Section 2 satisfies the hypotheses of Theorem 3.7, and we compute the first coefficient of its asymptotic expansion (3.37) in terms of the local data of Section 3.1.

We first work in the setting of Section 2.2, so that $\pi: M \rightarrow B$ is a prequantized fibration equipped with a relative compatible complex structure $J \in \operatorname{End}(T X)$ and an auxiliary vector bundle $\left(E, h^{E}, \nabla^{E}\right)$ over $M$. We assume $B$ compact and fix $p_{0} \in \mathbb{N}$ in Theorem 2.2 with $U=B$. Recall the $L^{2}$-connection $\nabla^{\mathscr{H}_{p}}$ on $\mathscr{H}_{p}$ of $(2.18)$, for any $p \in \mathbb{N}^{*}$. 
Definition 3.15. A family of connections $\left\{\nabla^{p}\right\}_{p \in \mathbb{N}^{*}}$ on the quantum bundle $\left\{\mathscr{H}_{p}\right\}_{p \in \mathbb{N}^{*}}$ over $B$ is called a Toeplitz connection if it is of the form

$$
\nabla^{p}=\nabla^{\mathscr{H}_{p}}+K_{p}
$$

for any $p \in \mathbb{N}$, with $\left\{K_{p} \in \mathscr{C}^{\infty}\left(B, \operatorname{End}\left(\mathscr{H}_{p}\right) \otimes T^{*} B\right)\right\}_{p \in \mathbb{N}^{*}}$ such that there exists a family $\left\{\sigma_{l} \in \mathscr{C}^{\infty}\left(M, \text { End }(E) \otimes T^{*} M\right)\right\}_{l \in \mathbb{N}}$ smooth in $t \in[0,1]$, such that for all $k \geqslant 0$ and any $v \in \mathscr{C}^{\infty}(B, T B)$, there exists $C_{k}>0$ such that for all $b \in B$,

$$
\left\|K_{p}(v)-\sum_{l=0}^{k-1} p^{-l} P_{p} \sigma_{l}\left(v^{H}\right) P_{p}\right\|_{p, b} \leqslant C_{k} p^{-k} .
$$

From now on, we fix a Toeplitz connection $\left\{\nabla^{p}\right\}_{p \in \mathbb{N}^{*}}$ and a path $\gamma:[0,1] \rightarrow B$. Let $p \in \mathbb{N}^{*}$, and recall that $\partial_{t}$ denotes the canonical vector field on $[0,1]$. The parallel transport along $\gamma$ with respect to $\left\{\nabla^{p}\right\}_{p \in \mathbb{N}^{*}}$ is the family of endomorphisms

$$
\mathcal{T}_{p, t}: \mathscr{H}_{p, \gamma(0)} \rightarrow \mathscr{H}_{p, \gamma(t)}
$$

satisfying the following differential equation in $t \in[0,1]$ for any $s_{0} \in \mathscr{H}_{p, \gamma(0)}$ and $p \in \mathbb{N}^{*}$,

$$
\left\{\begin{array}{l}
\nabla_{\partial_{t}}^{p} \mathcal{T}_{p, t} s_{0}=0 \\
\mathcal{T}_{p, 0} s_{0}=s_{0}
\end{array}\right.
$$

Pulling back the fibration by $\gamma:[0,1] \rightarrow B$, we can assume that $B=[0,1]$ and work in the setting of Section 2.3, where $\left(L, h^{L}, \nabla^{L}\right)$ is identified over $[0,1] \times X$ with the pullback of a fixed Hermitian bundle with connection over $X$. Then by (2.31), for any $t \in[0,1],(2.18)$ becomes

$$
\nabla_{\partial_{t}}^{\mathscr{H}_{p}}=P_{p, t} \nabla_{\partial_{t}^{H}}^{E} P_{p, t} .
$$

Let $\sigma_{0} \in \mathscr{C}^{\infty}\left(M, \operatorname{End}(E) \otimes T^{*} M\right)$ be the first coefficient of the Toeplitz connection $\left\{\nabla^{p}\right\}_{p \in \mathbb{N}^{*}}$ in (3.78), and define a connection $\nabla^{E, \sigma}$ on $E$ over $M$ by

$$
\nabla^{E, \sigma}=\nabla^{E}+\sigma_{0}
$$

For any $t \in[0,1]$, let $\tau_{t}^{E, \sigma}$ be the parallel transport with respect to $\nabla^{E, \sigma}$ along horizontal curves of $[0,1] \times X$. For any $t \in[0,1]$, let $\mu_{t} \in \mathscr{C}^{\infty}(X, \mathbb{C})$ be the function with value at $x_{0} \in X$ given by (3.18) in the trivialization of Section 2.3 around $x_{0} \in X$. The following theorem is the central result of this paper.

Theorem 3.16. There exists a family $\left\{\mu_{l, t} \in \mathscr{C}^{\infty}\left(X, E_{t} \otimes E_{0}^{*}\right)\right\}_{l \in \mathbb{N}}$, smooth in $t \in[0,1]$, such that for all $k \geqslant 0$, there exists $C_{k}>0$ such that

$$
\left\|\mathcal{T}_{p, t}-\sum_{l=0}^{k-1} p^{-l} P_{p, t} \mu_{l, t} P_{p, 0}\right\|_{p, 0, t} \leqslant C_{k} p^{-k},
$$

for all $p \in \mathbb{N}^{*}$ and $t \in[0,1]$. Furthermore, its first coefficient $\mu_{0, t} \in \mathscr{C}^{\infty}\left(X, E_{p, t} \otimes E_{p, 0}^{*}\right)$ is given by

$$
\mu_{0, t}=\mu_{t} \tau_{t}^{E, \sigma}
$$


Proof. For any $g_{t} \in \mathscr{C}^{\infty}\left(X, E_{t} \otimes E_{0}^{*}\right)$, smooth in $t \in[0,1]$, and for all $p \in \mathbb{N}^{*}$, let us consider the operator

$$
\nabla_{\partial_{t}}^{p} P_{p, t} g_{t} P_{p, 0}: \mathscr{H}_{p, 0} \rightarrow \mathscr{H}_{p, t}
$$

Then by (3.19), (3.34), (3.77) and (3.81), the Schwartz kernel of (3.85) satisfies the assumptions of Theorem 3.7. Using (3.82), the first coefficient $Q_{0, t, x_{0}}$ of its expansion (3.36) for any $x_{0} \in X, t \in[0,1]$, is the constant polynomial equal to

$$
\left(\nabla_{\partial_{t}^{H}}^{E, \sigma} g_{t}\right)\left(x_{0}\right)-\frac{1}{4} \operatorname{Tr}\left[\Pi_{t, x_{0}}^{0} \frac{\partial}{\partial t}\left(-J_{0, x_{0}} J_{t, x_{0}}\right)\right] g_{t}\left(x_{0}\right)
$$

where $\Pi_{t, x_{0}}^{0} \in \operatorname{End}\left(T_{x_{0}} X_{\mathbb{C}}\right)$ is the projection to $T_{x_{0}}^{(1,0)} X_{t}$ with kernel $T_{x_{0}}^{(0,1)} X_{0}$. Let $\mu_{0, t} \in$ $\mathscr{C}^{\infty}\left(X, E_{t} \otimes E_{0}^{*}\right)$ be the section satisfying the following ordinary differential equation in $t \in[0,1]$,

$$
\left\{\begin{array}{l}
\nabla_{\partial_{t}^{H}}^{E, \sigma} \mu_{0, t}-\frac{1}{4} \operatorname{Tr}\left[\Pi_{t}^{0} \frac{\partial}{\partial t}\left(-J_{0} J_{t}\right)\right] \mu_{0, t}=0 \\
\mu_{0,0}=\operatorname{Id}_{E_{0}} .
\end{array}\right.
$$

Then he have $P_{p, 0} \mu_{0,0} P_{p, 0}=P_{p, 0}$ for all $p \in \mathbb{N}^{*}$, and the estimate $\nabla_{\partial_{t}}^{p} P_{p, t} \mu_{0, t} P_{p, 0}=$ $O\left(p^{-1}\right)$ holds in operator norm as $p \rightarrow+\infty$ by (3.86). For any $k \in \mathbb{N}^{*}$, let us assume by induction that we have sections $\mu_{l, t} \in \mathscr{C}^{\infty}\left(X, E_{t} \otimes E_{0}^{*}\right)$ with $\mu_{l, 0} \equiv 0$ for all $0<l \leqslant k-1$, satisfying

$$
\nabla_{\partial_{t}}^{p} \sum_{l=0}^{k-1} p^{-l} P_{p, t} \mu_{l, t} P_{p, 0}=O\left(p^{-k}\right)
$$

in operator norm as $p \rightarrow+\infty$. Then Theorem 3.7 applies to the left hand side of (3.88) multiplied by $p^{k}$. Let $g_{k} \in \mathscr{C}^{\infty}\left(X, E_{t} \otimes E_{0}^{*}\right)$ denote its first coefficient in the expansion (3.37), and let $\mu_{k, t} \in \mathscr{C}^{\infty}\left(M, E_{t} \otimes E_{0}^{*}\right)$ be the section satisfying the following ordinary differential equation in $t \in[0,1]$,

$$
\left\{\begin{array}{l}
\nabla_{\partial_{t}}^{E, \sigma} \mu_{k, t}-\frac{1}{4} \operatorname{Tr}\left[\Pi_{t}^{0} \frac{\partial}{\partial t}\left(-J_{0} J_{t}\right)\right] \mu_{k, t}+g_{k}=0 \\
\mu_{k, 0}=0 .
\end{array}\right.
$$

Then using Theorem 3.7 as above, we have $\nabla_{\partial_{t}}^{p} \sum_{l=1}^{k} p^{-l} P_{p, t} \mu_{l, t} P_{p, 0}=O\left(p^{-k-1}\right)$ in operator norm as $p \rightarrow+\infty$. We construct this way a sequence $\left\{\mu_{l, t} \in \mathscr{C}^{\infty}\left(X, E_{p, t} \otimes E_{p, 0}^{*}\right)\right\}_{l \in \mathbb{N}}$, with $\mu_{0,0} \equiv \operatorname{Id}_{E}$ and $\mu_{l, 0} \equiv 0$ for all $l \in \mathbb{N}^{*}$, satisfying (3.88) for all $k \in \mathbb{N}^{*}$. Then by (3.80) and (3.88), we have $\nabla_{\partial_{t}}^{p}\left(\mathcal{T}_{p, t}-\sum_{l=1}^{k-1} p^{-l} P_{p, t} \mu_{l, t} P_{p, 0}\right)=O\left(p^{-k}\right)$ in operator norm as $p \rightarrow+\infty$ for any $k \in \mathbb{N}^{*}$, so that there exists $C_{k}>0$ such that for any $p \in \mathbb{N}^{*}$, 


$$
\begin{aligned}
& s_{0} \in \mathscr{C}^{\infty}\left(X, E_{p, 0}\right) \text { and } t \in[0,1] \\
& \frac{\partial}{\partial t}\left\|\left(\mathcal{T}_{p, t}-\sum_{l=1}^{k-1} p^{-l} P_{p, t} \mu_{l, t} P_{p, 0}\right) s_{0}\right\|_{p, t}^{2} \\
& \quad=2 \operatorname{Re}\left\langle\nabla_{\partial_{t}}^{p}\left(\mathcal{T}_{p}-\sum_{l=1}^{k-1} p^{-l} P_{p} \mu_{l, t} P_{p, 0}\right) s_{0},\left(\mathcal{T}_{p}-\sum_{l=1}^{k-1} p^{-l} P_{p, t} \mu_{l, t} P_{p, 0}\right) s_{0}\right\rangle_{p, t} \\
& \quad \leqslant 2 C_{k} p^{-k}\left\|s_{0}\right\|_{p, 0}\left\|\left(\mathcal{T}_{p}-\sum_{l=1}^{k} p^{-l} P_{p, t} \mu_{l, t} P_{p, 0}\right) s_{0}\right\|_{p, t} .
\end{aligned}
$$

As $\mathcal{T}_{p, 0}=\sum_{l=1}^{k-1} p^{-l} P_{p, 0} \mu_{l, 0} P_{p, 0}=P_{p, 0}$, by (3.90) and using Grönwall's lemma, we conclude that $\mathcal{T}_{p}$ satisfies (3.37), with first coefficient $\mu_{0, t}$ equal to the solution of the ordinary differential equation (3.86), which is precisely (3.84) by definition (3.18) of $\mu_{t}$.

By Lemma 3.6, Theorem 3.16 implies in particular that for any $x_{0} \in X$, there exists a family $\left\{G_{r, x_{0}}\left(Z, Z^{\prime}\right) \in E_{1, x_{0}} \otimes E_{0, x_{0}}^{*}\right\}_{r \in \mathbb{N}}$ of polynomials in $Z, Z^{\prime} \in \mathbb{R}^{2 n}$ of the same parity as $r$ and smooth in $x_{0} \in X, t \in[0,1]$, such that

$$
\mathcal{T}_{p, t}\left(Z, Z^{\prime}\right) \cong p^{n} \sum_{r=0}^{\infty} G_{r, t, x_{0}} \mathscr{P}_{t, x_{0}} \mathscr{P}_{0}\left(\sqrt{p} Z, \sqrt{p} Z^{\prime}\right) p^{-\frac{r}{2}}+\mathscr{O}\left(p^{-\infty}\right)
$$

in the sense of Notation 2.8, with $G_{0, t, x_{0}}\left(Z, Z^{\prime}\right)=\mu_{t}\left(x_{0}\right) \tau_{t}^{E, \sigma}\left(x_{0}\right)$ for all $Z, Z^{\prime} \in \mathbb{R}^{2 n}$.

\section{Localization formulas}

In this section, we use the results of Section 3.3 to prove an asymptotic version of localization formulas of Lefschetz type for the action of symplectic maps, proving Theorem 1.2. In Section 4.1, we deal with the case of isolated fixed point. In Section 4.2, we then establish the general case.

Let $(X, \omega)$ be a compact symplectic manifold, and let $\varphi: X \rightarrow X$ be a diffeomorphism.

Definition 4.1. The fixed point set $X^{\varphi} \subset X$ of a diffeomorphism $\varphi: X \rightarrow X$ is said to be non degenerate if $X^{\varphi}$ is a closed submanifold such that $T X^{\varphi}=\operatorname{Ker}\left(\operatorname{Id}_{T X}-d \varphi\right)$ inside $T X$.

Assume that $(X, \omega)$ is equipped with a Hermitian line bundle $\left(L, h^{L}\right)$ together with a Hermitian connection $\nabla^{L}$ whose curvature satisfies the prequantization condition (1.1), and that $\varphi: X \rightarrow X$ lifts to a bundle map $\varphi^{L}: L \rightarrow L$ preserving metric and connection, so that in particular $\varphi$ preserves $\omega$. If $J_{0}$ is an almost complex structure on $X$ compatible with $\omega$, then the almost complex structure $J_{1}$ defined by

$$
J_{1}=d \varphi J_{0} d \varphi^{-1}
$$


is again compatible with $\omega$. As the space of almost complex structures compatible with $\omega$ is contractible, there exists a path $t \mapsto J_{t}$ joining $J_{0}$ to $J_{1}$ for $t \in[0,1]$, and we can consider the associated tautological fibration over [0,1] as in Section 2.3. If $\left(E, h^{E}, \nabla^{E}\right)$ is an auxiliary Hermitian vector bundle with Hermitian connection over $[0,1] \times X$, we suppose that $\varphi$ lifts to a bundle map $\varphi^{E}: E_{0} \rightarrow E_{1}$ over $X$, preserving metric and connection, and we write $\varphi_{p}$ for the induced map on $E_{p}$ for any $p \in \mathbb{N}^{*}$. The pullback of $s \in \mathscr{C}^{\infty}\left(X, E_{p}\right)$ by $\varphi_{p}$, defined for any $x \in X$ by

$$
\left(\varphi_{p}^{*} s\right)(x)=\varphi_{p}^{-1} \cdot s(\varphi(x))
$$

induces by restriction a linear map $\varphi_{p}^{*}: \mathscr{H}_{p, 1} \rightarrow \mathscr{H}_{p, 0}$ from the quantum space associated with $J_{1}$ to the one associated with $J_{0}$.

We omit in the sequel the subscript 1 for any object depending on $t$ evaluated at $t=1$, and consider the local endomorphisms of Section 3.1 as functions of $x_{0} \in X$ in the trivialization of Section 2.3. In particular, we write $\mu \in \mathscr{C}^{\infty}(X, \mathbb{C})$ for the function whose value at $x_{0} \in X$ is equal to $\mu_{1}$ in (3.18).

In this section, the notation $O\left(p^{-k}\right)$ is meant in its usual sense as $p$ tends to infinity, uniformly in $x \in X$ and $t \in[0,1]$. The notation $O\left(p^{-\infty}\right)$ means $O\left(p^{-k}\right)$ for all $k \in \mathbb{N}$.

\subsection{Isolated fixed points}

We first deal with the case of $\varphi: X \rightarrow X$ having only non-degenerate isolated fixed points. Recall the parallel transport operator $\left\{\mathcal{T}_{p, t}\right\}_{p \in \mathbb{N}^{*}}$ from $\mathscr{H}_{p, 0}$ to $\mathscr{H}_{p, t}$ with respect to a Toeplitz connection $\left\{\nabla^{p}\right\}_{p \in \mathbb{N}}$ defined by (3.80) for all $t \in[0,1]$.

For any $x \in X$ such that $\varphi(x)=x$, write $\lambda_{x}:=\varphi_{x}^{L,-1} \in \mathbb{C}$ through the canonical identification $\operatorname{End}(L) \simeq \mathbb{C}$. Recall the convention for square roots of complex determinants stated at the end of Section 2.1. The following theorem is the main result of this section.

Theorem 4.2. Suppose that the fixed point set $X^{\varphi} \subset X$ of $\varphi: X \rightarrow X$ is discrete and non-degenerate, and write $X^{\varphi}=\left\{x_{1}, \ldots, x_{q}\right\}, q \in \mathbb{N}^{*}$. Then for each $1 \leqslant j \leqslant q$ and $r \in \mathbb{N}$, there exists $a_{j, r} \in \mathbb{C}$, such that for any $k \in \mathbb{N}$ and as $p \in \mathbb{N}^{*}$ tends to infinity,

$$
\operatorname{Tr}_{\mathscr{H}_{p}}\left[\varphi_{p}^{*} \mathcal{T}_{p}\right]=\sum_{j=1}^{q} \lambda_{x_{j}}^{p} \sum_{r=0}^{k-1} p^{-r} a_{j, r}+O\left(p^{-k}\right)
$$

Furthermore, for any $1 \leqslant j \leqslant q$, the following formula holds,

$$
a_{j, 0}=\bar{\mu}^{-1}\left(x_{j}\right) \operatorname{Tr}_{E}\left[\varphi_{x_{j}}^{E,-1} \tau_{x_{j}}^{E, \sigma}\right] \operatorname{det}^{-\frac{1}{2}}\left[\left(\Pi_{0, x_{j}}^{1}-d \varphi_{x_{j}}^{-1} \overline{\Pi_{1, x_{j}}^{0}}\right)\left(\operatorname{Id}_{T_{x_{j}} X}-d \varphi_{x_{j}}\right)\right] .
$$

Proof. Recall that $\mathcal{T}_{p}$ admits a smooth Schwartz kernel with respect to $d v_{X}$ for all $p \in \mathbb{N}^{*}$ and $t \in[0,1]$, so that in particular,

$$
\operatorname{Tr}_{\mathscr{H}_{p}}\left[\varphi_{p}^{*} \mathcal{T}_{p}\right]=\int_{X} \operatorname{Tr}_{E_{p}}\left[\varphi_{p}^{-1} \cdot \mathcal{T}_{p}(\varphi(x), x)\right] d v_{X}(x)
$$


Let $\psi: T X \rightarrow[0,1] \times X$ be a smooth family of vertical charts constant along $[0,1]$ as in Section 2.3, and let $\varepsilon_{0}>0$ be such that $\psi$ restricted to $B^{T_{x} X}\left(0, \varepsilon_{0}\right)$ is a diffeomorphism on its image for any $x \in X$. For all $x \in X$ and $0<\varepsilon<\varepsilon_{0}$, we write $U_{x}(\varepsilon):=$ $\psi\left(B^{T_{x} X}(0, \varepsilon)\right)$ and we identify $Z \in B^{T_{x} X}(0, \varepsilon)$ with its image in $U_{x_{j}}\left(\varepsilon_{0}\right)$.

Let $\eta \in \mathbb{R}$ be the modulus of the smallest eigenvalue of $\operatorname{Id}_{T_{x_{j}} X}-d \varphi_{x_{j}} \in \operatorname{End}\left(T_{x_{j}} X\right)$ for any $1 \leqslant j \leqslant q$. Then by Definition 4.1, we know that $|\eta|>0$. Let us now consider $\varepsilon>0$ small enough so that $\varphi\left(U_{x_{j}}(\varepsilon)\right) \subset U_{x_{j}}\left(\varepsilon_{0}\right)$, for all $1 \leqslant j \leqslant q$. Then taking the Taylor expansion of $\varphi$, we get $C_{j}>0$ such that for any $p \in \mathbb{N}^{*}, \theta \in[0,1]$ and all $Z \in B^{T_{x_{j}} X}(0, \varepsilon) \simeq U_{x_{j}}(\varepsilon)$ outside $U_{x_{j}}\left(\varepsilon p^{-\frac{\theta}{2}}\right)$, we have

$$
\begin{aligned}
|Z-\varphi(Z)| & \geqslant\left|\left(\operatorname{Id}_{T_{x_{j}} X}-d \varphi_{x_{j}}\right) Z\right|-C|Z|^{2} \\
& \geqslant\left(\eta-C \varepsilon p^{-\frac{\theta}{2}}\right) \varepsilon p^{-\frac{\theta}{2}} .
\end{aligned}
$$

On the other hand, by Theorem 3.16, we know that (2.25) holds for $\mathcal{T}_{p}$, and we deduce from (4.5)-(4.6) that there exists $\varepsilon>0$ such that for all $p \in \mathbb{N}^{*}$,

$$
\operatorname{Tr}_{\mathscr{H}_{p}}\left[\varphi_{p}^{*} \mathcal{T}_{p}\right]=\sum_{j=1}^{q} \int_{U_{x_{j}}\left(\varepsilon p^{-\frac{\theta}{2}}\right)} \operatorname{Tr}_{E_{p}}\left[\varphi_{p}^{-1} \cdot \mathcal{T}_{p}(\varphi(x), x)\right] d v_{X}(x)+O\left(p^{-\infty}\right)
$$

Estimating (4.7) term by term, we assume from now on that $\varphi$ has only one fixed point, which we denote $x_{0} \in X$. Consider the trivialization around $x_{0}$ by parallel transport with respect to $\nabla^{L}, \nabla^{E}$ along radial lines as in Section 2.3, and identify $T_{x_{0}} X$ with $\mathbb{R}^{2 n}$ by (2.32). Given any $s \in \mathscr{C}^{\infty}\left(U_{x_{0}}\left(\varepsilon_{0}\right), L\right)$, there exists a smooth function $\lambda_{x_{0}} \in \mathscr{C}^{\infty}\left(B^{\mathbb{R}^{2 n}}(0, \varepsilon), \mathbb{C}\right)$, defined for all $Z \in B^{\mathbb{R}^{2 n}}(0, \varepsilon)$ in these coordinates by the formula

$$
\lambda_{x_{0}}(Z) s(Z)=\left(\varphi^{L, *} s\right)(Z):=\varphi_{Z}^{L,-1} \cdot s(\varphi(Z)) .
$$

In particular, as $\varphi\left(x_{0}\right)=x_{0}$, the unitary endomorphism $\varphi_{x_{0}}^{L,-1}$ acts on $L_{x_{0}}$ by multiplication by $\lambda:=\lambda_{x_{0}}(0) \in \mathbb{C}$ with $|\lambda|=1$.

Recall that for any $v \in \mathscr{C}^{\infty}(X, T X)$ and $s \in \mathscr{C}^{\infty}(X, L)$, we have by assumption,

$$
\nabla_{v}^{L}\left(\varphi^{L, *} s\right)=\varphi^{L, *}\left(\nabla_{v}^{L} s\right)
$$

Let $Z:=\sum_{j=1}^{2 n} Z_{j} \frac{\partial}{\partial Z_{j}} \in \mathscr{C}^{\infty}\left(B^{\mathbb{R}^{2 n}}(0, \varepsilon), T_{x_{0}} X\right)$ be the radial vector field of the coordinates above. It is a classical result, which can be found for example in [41, (1.2.31)], that in the trivialization of $L$ along radial lines, the connection $\nabla^{L}$ at $Z \in B^{\mathbb{R}^{2 n}}(0, \varepsilon)$ has the form

$$
\nabla^{L}=d+\frac{1}{2} R^{L}(Z, .)+O\left(|Z|^{2}\right)
$$

Let us take $s \in \mathscr{C}^{\infty}\left(U_{x_{0}}\left(\varepsilon_{0}\right), L\right)$ in $(4.8)$ to be $1 \in \mathbb{C}$ in our trivialization. Then $s$ is parallel along radial lines, and the right hand side of (4.9) vanishes for $v=Z$. Thus using (4.8) and (4.10), equation (4.9) becomes

$$
\begin{aligned}
Z_{j} \frac{\partial}{\partial Z_{j}} \lambda_{x_{0}}(Z) & =-\frac{1}{2} R^{L}(Z, Z) \lambda_{x_{0}}(Z)+O\left(|Z|^{3}\right) \lambda_{x_{0}}(Z) \\
& =O\left(|Z|^{3}\right) \lambda_{x_{0}}(Z)
\end{aligned}
$$


Solving the ordinary differential equation (4.11), we get a function $\zeta \in \mathscr{C}^{\infty}\left(B^{\mathbb{R}^{2 n}}(0, \varepsilon), \mathbb{C}\right)$ such that for all $Z \in B^{\mathbb{R}^{2 n}}(0, \varepsilon)$,

$$
\lambda_{x_{0}}(Z)=\lambda e^{\zeta(Z)} \quad \text { with } \quad \zeta(Z)=O\left(|Z|^{3}\right) .
$$

Let $\left\{G_{r, x_{0}}\left(Z, Z^{\prime}\right) \in E_{1, x_{0}} \otimes E_{0, x_{0}}^{*}\right\}_{r \in \mathbb{N}}$ be the sequence of polynomials in $Z, Z^{\prime} \in \mathbb{R}^{2 n}$ associated with $\left\{\mathcal{T}_{p}\right\}_{p \in \mathbb{N}^{*}}$ in (3.91). By (4.7), (4.8) and (4.12), for any $\left.\delta \in\right] 0,1[$ and $k \in \mathbb{N}$, we get a $\theta \in] 0,1\left[\right.$ such that for all $p \in \mathbb{N}^{*}$,

$$
\begin{array}{r}
\operatorname{Tr}_{\mathscr{H}_{p}}\left[\varphi_{p}^{*} \mathcal{T}_{p}\right]=p^{n} \lambda^{p} \sum_{r=0}^{k-1} p^{-\frac{r}{2}} \int_{B^{\mathbb{R} 2 n}\left(0, \varepsilon p^{\left.-\frac{\theta}{2}\right)}\right.} \operatorname{Tr}_{E}\left[\varphi_{Z}^{E,-1} G_{r, x_{0}} \mathscr{P}_{1, x_{0}} \mathscr{P}_{0}(\sqrt{p} \varphi(Z), \sqrt{p} Z)\right] \\
e^{p \zeta(Z)} d v_{X}(Z)+O\left(p^{-\frac{k}{2}+\delta}\right)
\end{array}
$$

Recall (3.11). Considering the Taylor expansion up to second order of $\varphi$, we know there exist smooth functions $h_{\alpha} \in \mathscr{C}^{\infty}\left(B^{\mathbb{R}^{2 n}}(0, \varepsilon), \mathbb{C}\right)$ for all $\alpha \in \mathbb{N}^{3}$ such that for any $Z \in B^{\mathbb{R}^{2 n}}(0, \varepsilon)$,

$$
\begin{aligned}
\frac{\pi}{2}\left(\left\langle\Pi_{0, x_{0}}^{1}(\varphi(Z)-Z),(\varphi(Z)-Z)\right\rangle+2 \sqrt{-1} \Omega(\varphi(Z), Z)\right) \\
=\frac{\pi}{2}\left(\left\langle\Pi_{0, x_{0}}^{1}\left(d \varphi_{x_{0}} \cdot Z-Z\right),\left(d \varphi_{x_{0}} \cdot Z-Z\right)\right\rangle+2 \sqrt{-1} \Omega\left(d \varphi_{x_{0}} \cdot Z, Z\right)\right) \\
+\sum_{|\alpha|=3} Z^{\alpha} h_{\alpha}(Z) .
\end{aligned}
$$

Using (2.22) and (4.14), we then get for any $Z \in B^{\mathbb{R}^{2 n}}(0, \varepsilon), k, p \in \mathbb{N}^{*}$,

$$
\begin{aligned}
\mathscr{P}_{1, x_{0}} & \mathscr{P}_{0}(\sqrt{p} \varphi(Z), \sqrt{p} Z) \\
= & \mathscr{P}_{1, x_{0}} \mathscr{P}_{0}\left(\sqrt{p} d \varphi_{x_{0}} \cdot Z, \sqrt{p} Z\right) \exp \left(-p \sum_{|\alpha|=3} Z^{\alpha} h_{\alpha}(Z)\right) \\
= & \mathscr{P}_{1, x_{0}} \mathscr{P}_{0}\left(\sqrt{p} d \varphi_{x_{0}} \cdot Z, \sqrt{p} Z\right) \\
& \left(\prod_{|\alpha|=3} \sum_{r=0}^{k-1}(-1)^{r} \frac{p^{-\frac{r}{2}}}{r !}(\sqrt{p} Z)^{r \alpha} h_{\alpha}(Z)^{r}+p^{-\frac{k}{2}} O\left(|\sqrt{p} Z|^{k}\right)\right) .
\end{aligned}
$$

Now for any multi-index $\alpha$ of length 3 and any $r \in \mathbb{N}$, we can consider Taylor expansion up to order $l \in \mathbb{N}$ of $h_{\alpha}^{r}$ as in (3.53) to get $a_{\beta} \in \mathbb{C}$ for all $\beta \in \mathbb{N}^{2 n}$ such that for any $Z \in B^{\mathbb{R}^{2 n}}(0, \varepsilon), k \in \mathbb{N}$ and $p \in \mathbb{N}^{*}$,

$$
\begin{aligned}
\mathscr{P}_{1, x_{0}} \mathscr{P}_{0}(\sqrt{p} \varphi(Z), \sqrt{p} Z)= & \mathscr{P}_{1, x_{0}} \mathscr{P}_{0}\left(\sqrt{p} d \varphi_{x_{0}} \cdot Z, \sqrt{p} Z\right) \\
& \left(1+\sum_{j=1}^{k-1} p^{-\frac{j}{2}} \sum_{|\beta|=j}(\sqrt{p} Z)^{\beta} a_{\beta}+p^{-\frac{k}{2}} O\left(|\sqrt{p} Z|^{k}\right)\right) .
\end{aligned}
$$


Considering again the Taylor expansion of $\varphi$, for any polynomial $G_{r, x_{0}}\left(Z, Z^{\prime}\right)$ in (4.7), we get a sequence $\left\{F_{r, j}(Z) \in E_{1, x_{0}} \otimes E_{0, x_{0}}^{*}\right\}_{j \in \mathbb{N}}$ of polynomials in $Z \in \mathbb{R}^{2 n}$ of the parity of $r+j$ and some $d_{k} \in \mathbb{N}$, such that

$$
G_{r, x_{0}}\left(\sqrt{p} \varphi^{-1}(Z), \sqrt{p} Z\right)=\sum_{j=0}^{k-1} p^{-\frac{j}{2}} F_{r, j}(\sqrt{p} Z)+p^{-\frac{k}{2}} O\left(|\sqrt{p} Z|^{d_{k}}\right) .
$$

Furthermore, by (3.84) we get that $F_{0,0}(Z)=\mu\left(x_{0}\right) \tau_{x_{0}}^{E, \sigma}$ for any $Z \in \mathbb{R}^{2 n}$. From (3.11) and (4.6), we see that the function $\mathscr{P}_{1, x_{0}} \mathscr{P}_{0}\left(d \varphi_{x_{0}} . Z, Z\right)$ is integrable over $\mathbb{R}^{2 n}$ and exponentially decreasing in $Z \in \mathbb{R}^{2 n}$. For any $k \in \mathbb{N}$, let $M_{k} \in \mathbb{N}$ be the sum of the degrees of all polynomials involved in (4.16) and (4.17), and write

$$
\delta^{\prime}=\delta+\left(M_{k}+k+1+d_{k}\right)(1-\theta) / 2 .
$$

Then considering the Taylor expansion of $\varphi^{E,-1}$ in (4.13), applying the reasonning of (4.15)-(4.16) to $e^{p \zeta(Z)}$ with $\zeta(Z)=O\left(|Z|^{3}\right)$ as in (4.12) and using (4.16)-(4.17), we deduce the existence of a sequence $\left\{H_{r, x_{0}}(Z) \in E_{1, x_{0}} \otimes E_{0, x_{0}}^{*}\right\}_{r \in \mathbb{N}}$ of polynomials in $Z \in \mathbb{R}^{2 n}$, of the parity of $r$, smooth in $x_{0}$ and with $H_{0, x_{0}}(Z)=\mu\left(x_{0}\right) \tau_{x_{0}}^{E, \sigma}$ for any $Z \in \mathbb{R}^{2 n}$, such that via the change of variable $Z \mapsto \sqrt{p} Z$, equation (4.13) becomes

$$
\begin{aligned}
\operatorname{Tr}_{\mathscr{H}_{p}}\left[\varphi_{p}^{*} \mathcal{T}_{p}\right]= & \lambda^{p} \sum_{r=0}^{k-1} p^{-\frac{r}{2}} \\
& \int_{\mathbb{R}^{2 n}} \operatorname{Tr}_{E}\left[\varphi_{x_{0}}^{E,-1} H_{r, x_{0}} \mathscr{P}_{1, x_{0}} \mathscr{P}_{0}\left(\sqrt{p} d \varphi_{x_{0}} . Z, \sqrt{p} Z\right)\right] d Z+O\left(p^{-\frac{k}{2}+\delta^{\prime}}\right) .
\end{aligned}
$$

Now by (3.11), we see that $\mathscr{P}_{1, x_{0}} \mathscr{P}_{0}\left(\sqrt{p} d \varphi_{x_{0}} . Z, \sqrt{p} Z\right)$ is even in $Z \in \mathbb{R}^{2 n}$, and as the polynomial $H_{2 r+1}$ is odd for any $r \in \mathbb{N}$, we get

$$
\int_{\mathbb{R}^{2 n}} \operatorname{Tr}_{E}\left[\varphi_{x_{0}}^{E,-1} H_{2 r+1, t} \mathscr{P}_{1, x_{0}} \mathscr{P}_{0}\left(\sqrt{p} d \varphi_{x_{0}} . Z, \sqrt{p} Z\right)\right] d Z=0 .
$$

Thus from (4.19) and (4.20), we get $b_{r} \in \mathbb{C}$ for all $r \in \mathbb{N}$ such that

$$
\operatorname{Tr}_{\mathscr{H}_{p}}\left[\varphi_{p}^{*} \mathcal{T}_{p}\right]=\sum_{r=0}^{k-1} p^{-r} \lambda^{p} b_{r}+O\left(p^{-\frac{k}{2}+\delta}\right)
$$

Using (4.7) and (4.21), we can extend the above reasonning to the case of $m$ fixed point to get (4.3) in general.

Let us now compute

$$
b_{0, x_{0}}=\int_{\mathbb{R}^{2 n}} \operatorname{Tr}_{E}\left[\varphi_{x_{0}}^{E,-1} \tau_{x_{0}}^{E, \sigma}\right] \mu\left(x_{0}\right) \mathscr{P}_{1, x_{0}} \mathscr{P}_{0}\left(d \varphi_{x_{0}} . Z, Z\right) d Z .
$$

Using (3.7)-(3.10), we know that $\Pi_{0}^{1}+\overline{\Pi_{1}^{0}}=\operatorname{Id}_{\mathbb{R}^{2 n}}$ and that $\Omega\left(\Pi_{0}^{1} \cdot, \cdot\right)=\Omega\left(\cdot, \overline{\Pi_{1}^{0}} \cdot\right)$. From (3.9), (3.11), using $\Omega(\cdot, \cdot)=\Omega\left(d \varphi_{x_{0}} \cdot d \varphi_{x_{0}} \cdot\right)$ and the identities above, for any $Z, Z^{\prime} \in \mathbb{R}^{2 n}$ 
we get

$$
\begin{aligned}
& \operatorname{det}\left(A_{1}^{0}\right)^{-\frac{1}{2}} \mathscr{P}_{1, x_{0}} \mathscr{P}_{0}\left(d \varphi_{x_{0}} \cdot Z, Z\right) \\
& =\exp \left[-\pi\left(\left\langle\Pi_{0, x_{0}}^{1}\left(d \varphi_{x_{0}} \cdot Z-Z\right),\left(d \varphi_{x_{0}} \cdot Z-Z\right)\right\rangle+\sqrt{-1} \Omega\left(d \varphi_{x_{0}} \cdot Z, Z\right)\right)\right] \\
& =\exp \left[\sqrt{-1} \pi\left(\Omega\left(\left(\Pi_{0, x_{0}}^{1} d \varphi_{x_{0}}-\Pi_{0, x_{0}}^{1}\right) \cdot Z, d \varphi_{x_{0}} \cdot Z-Z\right)+\Omega\left(Z, d \varphi_{x_{0}} \cdot Z-Z\right)\right)\right] \\
& =\exp \left[\sqrt{-1} \pi\left(\Omega\left(Z,\left(d \varphi_{x_{0}}^{-1} \overline{\Pi_{1, x_{0}}^{0}}+\Pi_{0, x_{0}}^{1}\right)\left(d \varphi_{x_{0}} \cdot Z-Z\right)\right)\right)\right] \\
& =\exp \left[-\pi\left\langle\left(\Pi_{0, x_{0}}^{1}-d \varphi_{x_{0}}^{-1} \overline{\Pi_{1, x_{0}}^{0}}\right)\left(\operatorname{Id}_{\mathbb{R}^{2 n}}-d \varphi_{x_{0}}\right) \cdot Z, Z\right\rangle\right] .
\end{aligned}
$$

For the last line, we used the fact that $J_{0} d \varphi_{x_{0}}^{-1}=d \varphi_{x_{0}}^{-1} J_{1}$ by definition. Using (3.24) as in the proof of (3.21), the formula (4.22) then becomes

$$
\begin{aligned}
b_{0, x_{0}}=\bar{\mu}^{-1}\left(x_{0}\right) \operatorname{Tr}_{E}\left[\varphi_{x_{0}}^{E,-1} \tau_{x_{0}}^{E, \sigma}\right] & \\
& \int_{\mathbb{R}^{2 n}} \exp \left[-\pi\left\langle\left(\Pi_{0, x_{0}}^{1}-d \varphi_{x_{0}}^{-1} \overline{\Pi_{1, x_{0}}^{0}}\right)\left(\operatorname{Id}_{\mathbb{R}^{2 n}}-d \varphi_{x_{0}}\right) Z, Z\right\rangle\right] d Z .
\end{aligned}
$$

Using once again the identities mentioned above, we see that the endomorphism inside the exponential of (4.24) is symmetric, and its real part is obviously positive as $\mathscr{P}_{1, x_{0}} \mathscr{P}_{0}(d \varphi \cdot Z, Z)$ decreases exponentially in $Z \in \mathbb{R}^{2 n}$. We can thus apply the classical formula for the Gaussian integral to get (4.4) from (4.24).

\subsection{Higher dimensional fixed points}

In this section, we extend the asymptotic expansion of Theorem 4.2 to the case when the fixed point set $X^{\varphi} \subset X$ of $\varphi$ is a submanifold of arbitrary dimension satisfying the non-degeneracy condition of Definition 4.1.

Let $N$ be a subbundle of $T X$ over $X^{\varphi}$ such that $\left.T X\right|_{X^{\varphi}}=N \oplus T X^{\varphi}$, and equip $N$ with the Euclidean metric $g^{N}=\left.g_{0}^{T X}\right|_{N}$. Let $|d v|_{N}$ be the density of $\left(N, g^{N}\right)$. We define a density $|d v|_{T X / N}$ over $X^{\varphi}$ by the formula

$$
|d v|_{T X}=|d v|_{N}|d v|_{T X / N}
$$

Note that if $N$ is the normal bundle of $X^{\varphi}$ in $X$, then $|d v|_{T X / N}$ is simply the Riemannian density of $\left(X^{\varphi},\left.g_{0}^{T X}\right|_{X^{\varphi}}\right)$. We write $P^{N}:\left.T X\right|_{X^{\varphi}} \rightarrow N$ for the orthogonal projection with respect to $g_{0}^{T X}$.

Theorem 4.3. Suppose that the fixed point set $X^{\varphi}$ of $\varphi: X \rightarrow X$ is non-degenerate, and write $X^{\varphi}=\coprod_{j=1}^{q} X_{j}^{\varphi}, q \in \mathbb{N}$, for its decomposition into connected components. Set $d_{j}=\operatorname{dim} X_{j}^{\varphi}$, and for any $1 \leqslant j \leqslant m$, let $\lambda_{j} \in \mathbb{C}$ be the constant value of $\varphi^{L,-1}$ restricted to $X_{j}^{\varphi}$. Then there are densities $\nu_{r}$ over $X^{\varphi}$ for any $r \in \mathbb{N}$ such that for any $k \in \mathbb{N}$ and as $p \rightarrow+\infty$,

$$
\operatorname{Tr}_{\mathscr{H}_{p}}\left[\varphi_{p}^{*} \mathcal{T}_{p}\right]=\sum_{j=1}^{q} p^{d_{j} / 2} \lambda_{j}^{p}\left(\sum_{r=0}^{k-1} p^{-r} \int_{X_{j}^{\varphi}} \nu_{r}(x)+O\left(p^{-k}\right)\right) .
$$


Furthermore, the following equality holds,

$$
\nu_{0}=\bar{\mu}^{-1} \operatorname{Tr}_{E}\left[\varphi^{E,-1} \tau^{E, \sigma}\right] \operatorname{det}_{N}^{-\frac{1}{2}}\left[P^{N}\left(\Pi_{0}^{1}-d \varphi^{-1} \overline{\Pi_{1}^{0}}\right)\left(\operatorname{Id}_{T X}-d \varphi\right) P^{N}\right]|d v|_{T X / N} .
$$

Proof. For any $1 \leqslant j \leqslant q$, take a tubular neighborhood $U_{j}$ of $X_{j}^{\varphi}$. Then by (2.25) and (3.91), we know that

$$
\operatorname{Tr}_{\mathscr{H}_{p}}\left[\varphi_{p}^{*} \mathcal{T}_{p}\right]=\sum_{j=1}^{q} \int_{U_{j}} \operatorname{Tr}_{E_{p}}\left[\varphi_{p}^{-1} \cdot \mathcal{T}_{p}(\varphi(x), x)\right] d v_{X}(x)+O\left(p^{-\infty}\right)
$$

Then as in the proof of Theorem 4.2, we can assume without loss of generality that $X^{\varphi}$ has only one connected component, and set $d=\operatorname{dim} X^{\varphi}$. Furthermore, as all the computations are local on $X^{\varphi}$, we can assume $X^{\varphi}$ oriented. Write $d v_{X^{\varphi}}$ for the Riemannian volume form of $\left(X^{\varphi},\left.g_{0}^{T X}\right|_{X^{\varphi}}\right)$.

Let $|\cdot|_{N}$ be the norm on the subbundle $\left.N \subset T X\right|_{X^{\varphi}}$ transverse to $T X^{\varphi}$ over $X^{\varphi}$ induced by $g^{N}=\left.g_{0}^{T X}\right|_{N}$ as above. Let $\psi$ be a smooth family of vertical charts constant along $[0,1]$ as in Section 2.3, and let $\varepsilon_{0}>0$ be such that $\psi$ restricted to the ball bundle $B^{N}\left(0, \varepsilon_{0}\right):=\left\{\left.w \in N|| w\right|_{N}<\varepsilon_{0}\right\}$ is a diffeomorphism on its image. Then $U\left(\varepsilon_{0}\right):=\psi\left(B^{N}\left(0, \varepsilon_{0}\right)\right)$ is a tubular neighborhood of $X^{\varphi}$ in $X$.

Let $d w$ be a Euclidean volume form on the fibres of $\left(N, g^{T N}\right)$ such that the volume form $d w d v_{X^{\varphi}}$ on the total space of $N$ is compatible with the orientation of $X^{\varphi}$. Let $d v_{T X / N}$ be the volume form over $X^{\varphi}$ such that for any $y \in X^{\varphi}$ and $w \in U_{y}\left(\varepsilon_{0}\right)$,

$$
d v_{X}(y, w)=h(y, w) d w d v_{X^{\varphi}}(y)
$$

for some function $h \in \mathscr{C}^{\infty}\left(U\left(\varepsilon_{0}\right), \mathbb{R}\right)$ satisfying $h(y, 0)=1$ for all $y \in X^{\varphi}$. Following the proof of Theorem 4.2, by Definition 4.1 and compactness of $X^{\varphi}$, we know that

$$
\inf _{w \in N,|w|_{N}=1}\left|\left(d \varphi-\operatorname{Id}_{T X}\right) w\right|_{g_{0}^{T X}}>0 .
$$

Let us consider $\varepsilon>0$ small enough so that $\varphi(U(\varepsilon)) \subset U\left(\varepsilon_{0}\right)$. As in (4.6), we get $\varepsilon^{\prime}>0$ such that for all $p \in \mathbb{N}^{*}$ and $x \in X \backslash U\left(\varepsilon p^{-\frac{\theta}{2}}\right)$, we have $\varphi(x) \in X \backslash U\left(\varepsilon^{\prime} p^{-\frac{\theta}{2}}\right)$. By Theorem 3.16 and (2.25) as in (4.7), for any $p \in \mathbb{N}^{*}$ we get

$$
\begin{array}{r}
\operatorname{Tr}_{\mathscr{H}_{p}}\left[\varphi_{p}^{*} \mathcal{T}_{p}\right]=\int_{U\left(\varepsilon p^{-\frac{\theta}{2}}\right)} \operatorname{Tr}_{E_{p}}\left[\varphi_{p}^{-1} \cdot \mathcal{T}_{p}(\varphi(x), x)\right] d v_{X}(x)+O\left(p^{-\infty}\right) \\
=\int_{y \in X^{\varphi}} \int_{B^{N_{y}\left(0, \varepsilon p^{\left.-\frac{\theta}{2}\right)}\right.}} \operatorname{Tr}_{E_{p}\left[\varphi_{p}^{-1} \cdot \mathcal{T}_{p}(\varphi(y, w),(y, w))\right]} \quad h(y, w) d w d v_{T X / N}(y)+O\left(p^{-\infty}\right)
\end{array}
$$

Recall that we assumed $X^{\varphi}$ connected. By (4.9), the unitary endomorphism $\varphi_{y}^{L,-1} \in$ $\operatorname{End}\left(L_{y}\right) \simeq \mathbb{C}$ identifies with a constant complex number $\lambda \in \mathbb{C}$ such that $|\lambda|=1$. Fix $x_{0} \in X^{\varphi}$. We will estimate the middle integral of the right hand side of (4.31) for $y=x_{0}$ in the coordinates and trivialization of Section 2.3 . From (3.91), (4.29) and following 
(4.8)-(4.13), we get a function $\zeta \in \mathscr{C}^{\infty}\left(B^{T_{x_{0}} X}(0, \varepsilon), \mathbb{C}\right)$ such that for any $\left.\delta \in\right] 0,1[$ and $k \in \mathbb{N}$, there is $\theta \in] 0,1\left[\right.$ such that for all $p \in \mathbb{N}^{*}$,

$$
\begin{aligned}
\int_{B^{N x_{0}}\left(0, \varepsilon p^{-\frac{\theta}{2}}\right)} \operatorname{Tr}_{E}\left[\varphi_{p, w}^{-1} \mathcal{T}_{p}(\varphi(w), w)\right] h\left(x_{0}, w\right) d w= \\
p^{n} \sum_{r=0}^{k-1} p^{-\frac{r}{2}} \lambda^{p} \int_{B^{N_{0}}\left(0, \varepsilon p^{-\frac{\theta}{2}}\right)} \operatorname{Tr}_{E}\left[\varphi_{w}^{E,-1} G_{r, x_{0}} \mathscr{P}_{1, x_{0}} \mathscr{P}_{0}(\sqrt{p} \varphi(w), \sqrt{p} w)\right] \\
e^{p \zeta(w)} d w+O\left(p^{-\frac{k}{2}+\delta}\right) .
\end{aligned}
$$

By the argument of the proof of Theorem 4.2, we deduce from (4.32) the existence of a sequence $\left\{H_{r, x_{0}}\left(Z, Z^{\prime}\right) \in E_{0, x_{0}} \otimes E_{1, x_{0}}^{*}\right\}_{r \in \mathbb{N}}$ of polynomials in $Z, Z^{\prime} \in \mathbb{R}^{2 n}$ of the same parity as $r$, depending smoothly in $x_{0} \in X$ and with $H_{0, x_{0}}\left(Z, Z^{\prime}\right)=\mu\left(x_{0}\right) \tau_{x_{0}}^{E, \sigma}$, such that for any $\delta \in] 0,1[$ and $k \in \mathbb{N}$, there is a $\theta \in] 0,1\left[\right.$ such that for all $p \in \mathbb{N}^{*}$,

$$
\begin{aligned}
& \int_{B^{N x_{0}}\left(0, \varepsilon p^{\left.-\frac{\theta}{2}\right)}\right.} \operatorname{Tr}_{E}\left[\varphi_{p, w}^{-1} \mathcal{T}_{p}(\varphi(w), w)\right] h\left(x_{0}, w\right) d w \\
& =p^{\frac{d}{2}} \sum_{r=0}^{k-1} p^{-\frac{r}{2}} \lambda^{p} \int_{N_{x_{0}}} \operatorname{Tr}_{E}\left[\varphi_{x_{0}}^{E,-1} H_{r, x_{0}} \mathscr{P}_{1, x_{0}} \mathscr{P}_{0}\left(d \varphi_{x_{0}} . w, w\right)\right] d w+p^{\frac{d}{2}} O\left(p^{-\frac{k}{2}+\delta}\right) .
\end{aligned}
$$

Recall that $\operatorname{Ker}\left(d \varphi_{x_{0}}-\operatorname{Id}_{T_{x_{0}} X}\right) \cap N_{x_{0}}=\{0\}$ by assumption, so that the integrand of (4.33) decreases exponentially in $w \in N_{x_{0}}$ by (3.11). Then repeating the arguments of (4.19)-(4.21) and by integrating with respect to $x_{0} \in X^{\varphi}$, we produce from (4.31) and (4.33) a sequence $\left\{\nu_{r}\right\}_{r \in \mathbb{N}}$ of densities over $X^{\varphi}$, such that under the assumption of a unique connected component of $X^{\varphi}$, we have

$$
\operatorname{Tr}_{\mathscr{H}_{p}}\left[\varphi_{p}^{*} \mathcal{T}_{p}\right]=\lambda^{p} p^{\frac{d}{2}} \sum_{r=0}^{k-1} p^{-r} \int_{X^{\varphi}} \nu_{r}+p^{\frac{d}{2}} O\left(p^{-\frac{k}{2}+\delta}\right)
$$

and for any $y \in X$,

$$
\nu_{0}(y)=\operatorname{Tr}_{E}\left[\varphi_{y}^{E,-1} \tau_{y}^{E, \sigma}\right] \mu(y)\left(\int_{N_{y}} \mathscr{P}_{1, y} \mathscr{P}_{0}\left(d \varphi_{y} \cdot w, w\right) d w\right)|d v|_{T X / N}(y) .
$$

Then the computation of (4.27) from (4.35) is analogous to (4.23). The case of multiple components follows by (4.28) and linearity.

\section{Applications}

In Section 5.1, we interpret the formulas found in Theorems 4.2 and 4.3, and show that they are compatible with the local equivariant index formula in the holomorphic case. In Section 5.2, we introduce the notion of a Hitchin connection, and relate it with the notion of Toeplitz connection introduced in Definition 3.15. In Section 5.3, we present an application to Witten's asymptotic expansion conjecture for the quantum representations of the mapping class group. 


\subsection{Geometric interpretation}

Recall from Section 2.1 that the relative canonical line bundle $K_{X}:=\operatorname{det}\left(T^{(1,0) *} X\right)$ of a prequantized fibration is endowed with the connection $\nabla^{K_{X}}$ induced by the vertical Levi-Civita connection $\nabla^{T X}$ defined by (2.3). In this section, we will make use of the isomorphism

$$
\begin{aligned}
& T^{(0,1)} X \longrightarrow T^{(1,0) *} X \\
& \bar{w} \longmapsto g^{T X}(\bar{w}, \cdot)
\end{aligned}
$$

of complex vector bundles over $X \times[0,1]$ induced by the relative Riemannian metric $g^{T X}$ seen as a $\mathbb{C}$-bilinear form over $T X_{\mathbb{C}}$. Via this isomorphism, the line bundle with connection $\left(K_{X}, \nabla^{K_{X}}\right)$ identifies with the line bundle $\operatorname{det}\left(T^{(0,1)} X\right)$ endowed with the connection $\nabla^{\operatorname{det}\left(T^{(0,1)} X\right)}$ induced by $\nabla^{T X}$. The following lemma gives a geometric interpretation of the function $\mu_{t} \in \mathscr{C}^{\infty}(X, \mathbb{C})$ defined by formula (3.18) in the local model, and allows to deduce Theorem 1.1 from Theorem 3.16.

Lemma 5.1. For any $t \in[0,1]$, we have following formula for the function $\mu_{t} \in$ $\mathscr{C}^{\infty}(X, \mathbb{C})$ appearing Theorem 3.16,

$$
\bar{\mu}_{t}^{2}=\operatorname{det}\left(\overline{\Pi_{t}^{0}}\right)^{-1} \tau_{t}^{K_{X}},
$$

where $\tau_{t}^{K_{X}}: K_{X, 0} \rightarrow K_{X, t}$ is the parallel transport in $K_{X}$ over horizontal lines of $[0,1] \times X$ with respect to $\nabla^{K_{X}}$ and $\operatorname{det}\left(\overline{\Pi_{t}^{0}}\right): K_{X, 0} \rightarrow K_{X, t}$ is the bundle isomorphism induced by $\overline{\Pi_{t}^{0}}$ via the isomorphism (5.1).

Proof. Consider the setting of Section 2.3. Using Koszul formula and the definition (2.3) of $\nabla^{T X}$, we know that

$$
\nabla_{\partial_{t}^{H}}^{T X}=\frac{\partial}{\partial t}+\frac{1}{2} J_{t}\left(\frac{\partial}{\partial t} J_{t}\right)
$$

in the tautological fibration $\pi:[0,1] \times X \rightarrow[0,1]$. Thus by $(2.5)$, for all $t \in[0,1]$ we have

$$
\nabla_{\partial_{t}^{H}}^{T^{(0,1)} X}=P_{t}^{(0,1)} \frac{\partial}{\partial t} P_{t}^{(0,1)}
$$

Recall the notations of Section 3.1, and for any $t \in[0,1]$, let $\overline{\Pi_{t}^{0}} \in \operatorname{End}\left(T X_{\mathbb{C}}\right)$ be the projection on $T^{(0,1)} X_{t}$ with kernel $T^{(1,0)} X_{0}$, which restricts to a natural bundle isomorphism between $T^{(0,1)} X_{0}$ and $T^{(0,1)} X_{t}$. Using (3.7)-(3.9), for any $w \in T^{(0,1)} X_{0}$, we compute

$$
\begin{aligned}
P_{t}^{(0,1)} \frac{\partial}{\partial t} P_{t}^{(0,1)} \overline{\Pi_{t}^{0}} \cdot w & =P_{t}^{(0,1)}\left(\frac{\partial}{\partial t} A_{t}^{0}\right) P_{0}^{(0,1)} \cdot w \\
& =\left(-\frac{1}{2} \overline{\Pi_{t}^{0}} \frac{\partial}{\partial t}\left(-J_{0} J_{t}\right)\right) \overline{\Pi_{t}^{0}} \cdot w .
\end{aligned}
$$

By the definition (3.18) of $\mu_{t}$ and via the isomorphism (5.1) induced by the relative Riemannian metric $g^{T X}$, this shows (5.2). 
Recall (3.82) and (4.25), and consider the context of Section 4. The following Lemma gives a geometric interpretation for the localization formula (4.27), and allows to deduce Theorem 1.2 from Theorem 4.3.

Lemma 5.2. Suppose that the tangent bundle $T X$ over the fixed point set $X^{\varphi}$ admits a decomposition $T X=T X^{\varphi} \oplus N$ preserved by $d \varphi$ and $J_{0}$. Then over any connected component of $X^{\varphi}$ of dimension $2 d$, the first coefficient (4.27) in Theorem 4.3 satisfies

$$
\nu_{0}=(-1)^{\frac{n-d}{2}} \operatorname{Tr}_{E}\left[\varphi^{E,-1} \tau^{E, \sigma}\right]\left(\varphi^{K_{X}} \tau^{K_{X},-1}\right)^{1 / 2}\left|\operatorname{det}_{N}\left(\operatorname{Id}_{T N}-\left.d \varphi\right|_{N}\right)\right|^{-\frac{1}{2}}|d v|_{T X / N},
$$

for some natural choice of square roots depending on $\varphi$ and the choice of the path.

Proof. Let $\varphi: X \rightarrow X$ be a diffeomorphism lifting to $\left(L, h^{L}, \nabla^{L}\right)$, sending $J_{0}$ to $J_{1}$ as in (4.1). Let $x \in X$ be a fixed point of $\varphi$. Using that $\Pi_{0, x}^{1}, \overline{\Pi_{1, x}^{0}} \in \operatorname{End}\left(T_{x} X_{\mathbb{C}}\right)$ are the projection operators on $T_{x}^{(1,0)} X_{0}, T_{x}^{(0,1)} X_{1}$ with kernel $T_{x}^{(0,1)} X_{1}, T_{x}^{(1,0)} X_{0}$, and that $d \varphi \cdot T_{x}^{(1,0)} X_{0}=T_{x}^{(1,0)} X_{1}$, we know that

$$
\begin{aligned}
& \left(\Pi_{0, x}^{1}-d \varphi_{x}^{-1} \overline{\Pi_{1, x}^{0}}\right) \cdot v=v \text { for any } v \in T_{x}^{(1,0)} X_{0}, \\
& \left(\Pi_{0, x}^{1}-d \varphi_{x}^{-1} \overline{\Pi_{1, x}^{0}}\right) d \varphi_{x} \cdot v=-v \text { for any } v \in T_{x}^{(0,1)} X_{0} .
\end{aligned}
$$

In particular, as $T^{(1,0)} X_{0}$ and $d \varphi \cdot T_{x}^{(1,0)} X_{0}=T_{x}^{(1,0)} X_{1}$ are transverse by (3.10), we get that $\left(\Pi_{0, x}^{1}-d \varphi_{x}^{-1} \overline{\Pi_{1, x}^{0}}\right)$ is invertible, and if $\left\{w_{j}\right\}_{j=1}^{n}$ is a basis of $T_{x}^{(1,0)} X_{0}$,

$$
\begin{aligned}
\operatorname{det}\left(\Pi_{0, x}^{1}-d \varphi_{x}^{-1} \bar{\Pi}_{1, x}^{0}\right)^{-1} & =\frac{w_{1} \wedge \cdots \wedge w_{n} \wedge\left(-d \varphi_{x}\right) \cdot \bar{w}_{1} \wedge \cdots \wedge\left(-d \varphi_{x}\right) \cdot \bar{w}_{n}}{w_{1} \wedge \cdots \wedge w_{n} \wedge \bar{w}_{1} \wedge \cdots \wedge \bar{w}_{n}} \\
& =(-1)^{n} \frac{d \varphi_{x} \cdot \bar{w}_{1} \wedge \cdots \wedge d \varphi_{x} \cdot \bar{w}_{n}}{\bar{\Pi}_{1, x}^{0} \bar{w}_{1} \wedge \cdots \wedge \bar{\Pi}_{1, x}^{0} \bar{w}_{n}} \\
& =(-1)^{n} \varphi_{x}^{K_{X}} \operatorname{det}\left(\overline{\Pi_{1}^{0}}\right)_{x}^{-1}
\end{aligned}
$$

with $\operatorname{det}\left(\overline{\Pi_{t}^{0}}\right): K_{X, 0} \rightarrow K_{X, t}$ as in Lemma 5.1 and $\varphi^{K_{X}}: K_{X, 0} \rightarrow K_{X, 1}$ the natural bundle map induced by $\varphi$ via the isomorphism (5.1), which commutes with the natural action of $\varphi$ as $g_{1}^{T X}(\cdot, \cdot)=g_{0}^{T X}(d \varphi \cdot d \varphi \cdot)$ by definition. Then in the notations of Section 4 , by (5.2) and (5.8) we get

$$
\begin{aligned}
\bar{\mu}^{-2}(x) \operatorname{det}\left(\Pi_{0, x}^{1}-d \varphi_{x}^{-1} \overline{\Pi_{1, x}^{0}}\right) & =(-1)^{n} \varphi_{x}^{K_{X}} \bar{\mu}^{-2}(x) \operatorname{det}\left(\overline{\Pi_{1}^{0}}\right)_{x}^{-1} \\
& =(-1)^{n} \varphi_{x}^{K_{X}}\left(\tau_{x}^{K_{X}}\right)^{-1} .
\end{aligned}
$$

Assume now that $d \varphi$ and $J_{0}$ preserve a decompostion $T X=T X^{\varphi} \oplus N$ over $X^{\varphi}$. Then by (5.7), we see that $\left(\Pi_{0}^{1}-d \varphi^{-1} \overline{\Pi_{1}^{0}}\right)$ over $X^{\varphi}$ preserves $N$ as well, and that its restriction to $N$ is invertible. Then by computations analogous to (5.8) and by (5.9), over any connected component of $X^{\varphi}$ of dimension $2 d$, we get

$$
\begin{aligned}
\bar{\mu}^{-2} \operatorname{det}_{N}\left[P ^ { N } \left(\Pi_{0}^{1}-\right.\right. & \left.\left.d \varphi^{-1} \overline{\Pi_{1}^{0}}\right)\left(\operatorname{Id}_{T X}-d \varphi\right) P^{N}\right]^{-1} \\
= & \bar{\mu}^{-2} \operatorname{det}_{N}\left[\left.\left(\Pi_{0}^{1}-d \varphi^{-1} \overline{\Pi_{1}^{0}}\right)\right|_{N}\right]^{-1} \operatorname{det}_{N}\left(\operatorname{Id}_{N}-\left.d \varphi\right|_{N}\right)^{-1} \\
= & (-1)^{n-d} \varphi^{K_{X}}\left(\tau^{K_{X}}\right)^{-1} \operatorname{det}_{N}\left(\operatorname{Id}_{N}-\left.d \varphi\right|_{N}\right)^{-1} .
\end{aligned}
$$


This together with (4.27) shows (5.6).

We see that formula (4.27) acquires a natural interpretation in terms of parallel transport in a square root of $K_{X}$, which always exists locally. In particular, if $c_{1}(T X)$ is even, so that we can take $E=: K_{X}^{1 / 2}$ whose square is equal to $K_{X}$ with induced metric and connection, and if there is a lift of $\varphi$ to $K_{X}^{1 / 2}$ squaring to $\varphi^{K_{X}}$ on $K_{X}$, Then (5.6) simplifies and we recover [27, Th.5.3.1] as a special case, when $(X, J, \omega)$ Kähler and $X^{\varphi}$ discrete. Consider now the context of Section 3.

Lemma 5.3. Let $\pi: B \times X \rightarrow B$ be a tautological fibration with relative complex structure $J \in \operatorname{End}(T X)$ and auxiliary vector bundle $\left(E, h^{E}, \nabla^{E}\right)$, and let $\gamma:[0,1] \rightarrow B$ be such that $\gamma(0)=\gamma(1)=b_{0} \in B$. Let $\mathcal{T}_{p} \in \operatorname{End}\left(\mathscr{H}_{p, b_{0}}\right)$ be the parallel transport in $\mathscr{H}_{p}$ along $\gamma$ with respect to a Toeplitz connection, for all $p \in \mathbb{N}^{*}$ big enough. Then there exists $C>0$ such that as $p \rightarrow+\infty$,

$$
\left\|\mathcal{T}_{p}-P_{p}\left(\tau^{K_{X}}\right)^{-\frac{1}{2}} \tau^{E, \sigma} P_{p}\right\|_{p, b_{0}}<C p^{-1}
$$

for an appropriate choice of square root of the parallel transport $\tau^{K_{X}} \in \operatorname{End}\left(K_{X}\right) \simeq$ $\mathscr{C}^{\infty}(X, \mathbb{C})$ in $K_{X}$ along $\gamma$.

Proof. Restricting the fibration over $\gamma$ as in Section 2.3, we see that the diffeomorphism $\tau_{1}: X_{0} \rightarrow X_{1}$ defined in (2.29) is the identity of $X \simeq X_{1}=X_{0}$, so that $J_{1}=J_{0}$, $P_{p, 1}=P_{p, 0}$ and $\operatorname{det}\left(\overline{\Pi_{1}^{0}}\right)=1$. Then (5.11) is a consequence of Theorem 3.16, comparing (3.84) with (5.2).

In the case $\gamma$ is contractible in $B$, if the fibration is holomorphic as in Proposition 2.3 and for the $L^{2}$-connection, this result is a consequence of the computations in [44, Th.2.1] of the curvature of $\nabla^{\mathscr{H}_{p}}$. Note that [44, Th.2.1] applies for general Kähler fibrations, which need not be tautological, and the same is true for Theorem 3.16. Taking $\varphi: X \rightarrow X$ to be the diffeomorphism identifying $X_{1}$ with $X_{0}$ via (2.29) on a loop $\gamma:[0,1] \rightarrow B$, we recover in general the first coefficient of $[44,(8)]$ as the contribution of $\varphi_{p}: L^{p} \rightarrow L^{p}$ through the description of the curvature as the derivative of the parallel transport.

\subsection{Hitchin connections}

In this section, we describe an important class of Toeplitz connections called Hitchin connections, which appear naturally in the context of geometric quantization of moduli spaces. These are the connections used in the application of Theorem 4.3 to Witten's asymptotic expansion conjecture, which we describe in Section 5.3.

Consider a holomorphic prequantized fibration $\pi: M \rightarrow B$, and fix $p_{0} \in \mathbb{N}$ as in Theorem 2.2 for $U=B$. Recall that $J \in \operatorname{End}(T X)$ over $M$ denotes the associated relative compatible complex structure. For any $v \in \mathscr{C}^{\infty}(B, T B)$, let $\tau_{t}^{v}$ be the flow on $M$ generated by its horizontal lift $v^{H} \in \mathscr{C}^{\infty}(M, T M)$ with respect to (2.2) at time 
$t \in \mathbb{R}$. Then $\tau_{t}^{v}$ preserves the fibres, so that its differential restricts to a bundle map $d \tau_{t}^{v}: T X \rightarrow T X$. Define the Lie derivative of $J$ by the formula

$$
L_{v} J=\left.\frac{\partial}{\partial t}\right|_{t=0} d \tau_{t}^{v} . J .\left(d \tau_{t}^{v}\right)^{-1} \in \operatorname{End}(T X)
$$

Then $L_{v} J \in \operatorname{End}(T X)$ exchanges $T^{(1,0)} X$ and $T^{(0,1)} X$ inside $T X$ as in (2.4). For any $w \in T X_{\mathbb{C}}$, recall that $\iota_{w} \in \operatorname{End}\left(\Lambda\left(T^{*} X_{\mathbb{C}}\right)\right)$ denotes the contraction by $w$. For any $w_{1}, w_{2} \in T X_{\mathbb{C}}$, we define

$$
\begin{aligned}
i_{w_{1} \otimes w_{2}}: \Lambda\left(T^{*} X_{\mathbb{C}}\right) & \rightarrow T X_{\mathbb{C}} \otimes \Lambda\left(T^{*} X_{\mathbb{C}}\right) \\
\alpha & \mapsto w_{1} \otimes \iota_{w_{2}} \alpha,
\end{aligned}
$$

and we extend this definition to all of $T X_{\mathbb{C}} \otimes T X_{\mathbb{C}}$ by linearity. Then for any $A \in$ $T X_{\mathbb{C}} \otimes T X_{\mathbb{C}}$, we can consider $i_{A} \omega \in T X_{\mathbb{C}} \otimes T^{*} X_{\mathbb{C}}$ as an element of $\operatorname{End}\left(T X_{\mathbb{C}}\right)$. Following $[2, \S 1]$, we define a section $G \in \mathscr{C}^{\infty}\left(M, \pi^{*} T^{*} B \otimes T^{(1,0)} X \otimes T^{(1,0)} X\right)$ by the following formula, for all $v \in T B$ and $w \in T^{(0,1)} X$,

$$
L_{v} J . w=2 \pi\left(i_{G(v)} \omega\right) . w .
$$

We still write $\nabla^{T^{(1,0)} X}$ for the connection on $T^{(1,0)} X \otimes T^{(1,0)} X$ induced by (2.5). The following definition can be found in [2, Def.1].

Definition 5.4. An holomorphic prequantized fibration is said to be rigid if for all $v \in \mathscr{C}^{\infty}(B, T B)$, we have

$$
\nabla^{T^{(1,0)} X} G(v)=0 .
$$

For any vector bundle with connection $\left(E, \nabla^{E}\right)$ over $M$ and any $A \in T^{(1,0)} M^{\otimes 2}$, let $\Delta_{A}^{E}$ be the second order differential operator in the fibres of $\pi: M \rightarrow B$, defined for any $w_{1}, w_{2} \in T^{(1,0)} X$ by the formula

$$
\Delta_{w_{1} \otimes w_{2}}^{E} s=\operatorname{Tr}\left[\nabla^{T^{(1,0)} X \otimes E}\left(w_{1} \otimes \nabla_{w_{2}}^{E} s\right)\right] .
$$

Recall (2.2), and suppose that there exists $k \in \mathbb{N}^{*}$ and a function $\rho \in \mathscr{C}^{\infty}(M, \mathbb{R})$ such that

$$
k \omega^{X}=\frac{\sqrt{-1}}{2 \pi}\left[\operatorname{Tr} R^{T^{(1,0)} X}+\bar{\partial} \partial \rho\right]^{X} .
$$

Using the notations of Section 2.1, we are now ready to state the following theorem, which is originally due to $[33, \S 3]$ and $[12, \S 4 . b]$. In this generality, it is due to [2, Th.1].

Theorem 5.5. Let $\pi: M=B \times X \rightarrow B$ be a rigid holomorphic tautological fibration with $E=\mathbb{C}$. Suppose that $X$ is simply connected and that (5.17) holds for some $\rho \in \mathscr{C}^{\infty}(X, \mathbb{R})$. For any $p \in \mathbb{N}^{*}$ and $v \in \mathscr{C}^{\infty}(B, T B)$, let $\nabla_{v}^{p}$ be the differential operator acting on $\mathscr{C}^{\infty}\left(M, L^{p}\right)$ by

$$
\nabla_{v}^{p}=\nabla_{v^{H}}^{L^{p}}+\frac{1}{4 p+2 k}\left(\Delta_{G(v)}^{L^{p}}-\nabla_{i_{G(v)} L^{p}}^{L^{2}}+2 p \partial \rho . v^{H}\right)
$$


Then $\nabla_{v}^{p}$ preserves holomorphicity in the fibres for any $v \in \mathscr{C}^{\infty}(B, T B)$ and $p \in \mathbb{N}^{*}$, and thus induces by restriction a family of connections $\left\{\nabla^{p}\right\}_{p \in \mathbb{N}^{*}}$ on the quantum bundle $\left\{\mathscr{H}_{p}\right\}_{p \in \mathbb{N}^{*}}$ over $B$.

In particular, using parallel transport with respect to (5.18), this shows that the dimension of the space of holomorphic sections is constant, so that the quantum bundle is well defined for any $p \in \mathbb{N}^{*}$. Recall Definition 3.15.

Lemma 5.6. Under the hypotheses of Theorem 5.5, the family of connections $\left\{\nabla^{p}\right\}_{p \in \mathbb{N}^{*}}$ defined by (5.18) is a Toeplitz connection. Furthermore, its first coefficient in (3.78) satisfies

$$
\sigma_{0}=\frac{1}{2} \partial \rho .
$$

Proof. By Theorem 5.5, the differential operator $\nabla_{v}^{p}$ defined in (5.18) for any $v \in$ $\mathscr{C}^{\infty}(B, T B)$ and $p \in \mathbb{N}^{*}$ preserves the fibrewise holomorphic sections, so that (5.18) rewrites

$$
\begin{aligned}
\nabla_{v}^{p} & =P_{p}\left[\nabla_{v^{H}}^{L_{p}}+\frac{1}{4 p+2 k}\left(\Delta_{G(v)}^{L^{p}}-\nabla_{i_{G(v)} d_{\rho}^{p}}^{L^{p}}+2 p \partial \rho . v^{H}\right)\right] P_{p} \\
& =\nabla_{v}^{\mathscr{H}_{p}}+\frac{1}{4 p+2 k} P_{p}\left(\Delta_{G(v)}^{L^{p}}-\nabla_{i_{G(v)} L^{p}}^{L^{p}}+2 p \partial \rho \cdot v^{H}\right) P_{p}
\end{aligned}
$$

Now by straightforward computations in the spirit of [49, Th.2.1], there exist functions $g, h \in \mathscr{C}^{\infty}(M, \mathbb{C})$ such that

$$
\begin{aligned}
P_{p} \Delta_{G(v)}^{L^{p}} P_{p} & =P_{p} g P_{p}, \\
P_{p} \nabla_{i_{G(v)} L_{\rho}}^{L^{p}} P_{p} & =P_{p} h P_{p} .
\end{aligned}
$$

We can then take the expansion in $p \in \mathbb{N}^{*}$ of the multiplicative constant in front of the second term of (5.20) to get the result by (3.78).

Let us now describe a variant of Theorem 5.5 including a square root of $K_{X}$, which is originally due to $[46, \S 3]$, and which in this generality is due to [4, Th.1.2].

Theorem 5.7. Under the assumptions of Theorem 5.5, with $c_{1}(T X)$ even and taking $E=K_{X}^{1 / 2}$, there exists a 1 -form $\beta \in \mathscr{C}^{\infty}\left(M, T^{*} M_{\mathbb{C}}\right)$ such that for any $p \in \mathbb{N}^{*}$ and $v \in \mathscr{C}^{\infty}(B, T B)$, the differential operator $\nabla_{v}^{p}$ acting on $\mathscr{C}^{\infty}\left(M, L^{p} \otimes K_{X}^{1 / 2}\right)$ defined by

$$
\nabla_{v}^{p}=\nabla_{v^{H}}^{E_{p}}+\frac{1}{4 p}\left(\Delta_{G(v)}^{E_{p}}+\beta\left(v^{H}\right)\right),
$$

preserves holomorphicity in the fibres, for any $v \in \mathscr{C}^{\infty}(B, T B)$ and $p \in \mathbb{N}^{*}$, and thus induces by restriction a family of connections $\left\{\nabla^{p}\right\}_{p \in \mathbb{N}^{*}}$ on the quantum bundle $\left\{\mathscr{H}_{p}\right\}_{p \in \mathbb{N}^{*}}$ over B.

We then have the following analogue of Lemma 5.6, whose proof is strictly analogous. 
Lemma 5.8. Under the hypotheses of Theorem 5.7, the family of connections $\left\{\nabla^{p}\right\}_{p \in \mathbb{N}^{*}}$ defined by (5.22) is a Toeplitz connection. Furthermore, its first coefficient in (3.78) satisfies $\sigma_{0}=0$.

Consider now a diffeomorphism $\varphi: X \rightarrow X$ lifting to a bundle map $\varphi: L \rightarrow L$ preserving metric and connection, and assume that the induced action of $\varphi$ on the space of compatible complex structures preserves $B$. Then by Lemma 5.6, if $\varphi$ has nondegenerate fixed point set, we can apply Theorem 4.3 to the situation of Theorem 5.5. We recover in this way the result of [6, Th.1.2], and moreover, we give an explicit formula for the first coefficient. On the other hand, by Lemma 5.8 we can apply Theorem 4.3 to the situation of Theorem 5.7, under the additional hypothesis that there exists a lift of $\varphi$ to $K_{X}^{1 / 2}$ squaring to $\varphi^{K_{X}}$. In the case $\operatorname{dim} X^{\varphi}=0$, this is the result of [28, Th.6.3, Th.6.4], with the corresponding formula (5.6) for the first coefficient.

\subsection{Witten's aymptotic conjecture}

In this section, we explain how the results of Section 4 apply to Witten's asymptotic expansion conjecture for quantum representation of the mapping class group described in the introduction.

Let $\Sigma$ be an oriented compact surface of genus $g \geqslant 2$, and let $P$ be the trivial $\mathrm{SU}(m)$-principal bundle over $\Sigma$, with $m \geqslant 2$. Let $D$ be a disk inside $\Sigma$, and choose $d \in(\mathbb{Z} / m \mathbb{Z})^{*}$. We consider the space $\mathscr{A}$ of flat connections on $P$ over $\Sigma \backslash D$ with holonomy along its boundary equal to $e^{\frac{2 \pi \sqrt{-1} d}{m}} \operatorname{Id}_{\mathbb{C}^{m}} \in \mathrm{SU}(m)$. The gauge group $\operatorname{Aut}(P)$ of automorphisms of $P$ acts naturally on $\mathscr{A}$, and it is a basic fact that all $A \in \mathscr{A}$ are irreducible, meaning that the stabiliser of $A$ in $\operatorname{Aut}(P)$ is the center of $\operatorname{Aut}(P)$, which identifies with the center of $\mathrm{SU}(m)$.

Let $\mathscr{A}^{\prime} \subset \mathscr{A}$ be the connections with some fixed standard form in a neighbourhood of the boundary of $\Sigma \backslash D$, and note that any $[A] \in \mathscr{A} / \operatorname{Aut}(P)$ has a representative in $\mathscr{A}^{\prime}$. If $t \mapsto A_{t} \in \mathscr{A}^{\prime}$ is smooth in $t \in \mathbb{R}$, we can extend $\left.\frac{d}{d t}\right|_{t=0} A_{t}$ by 0 over $D$ and consider it as an element of $\Omega^{1}(\Sigma, \mathfrak{s u}(m))$. Any $A \in \mathscr{A}^{\prime}$ induces a flat connection on the trivial adjoint bundle ad $P$ over $\Sigma \backslash D$ with trivial holonomy along the boundary, so that it extends to a covariant derivative $d_{A}$ on $\Omega^{\bullet}(\Sigma, \mathfrak{s u}(m))$ satisfying $d_{A}^{2}=0$. Let $H_{A}^{\bullet}(\Sigma)$ denote its cohomology. The following result is classical and is essentially due to [10, p. 587, Th.9.12, §14] and [31, §1.2, 1.8$]$.

Proposition 5.9. The quotient $\mathscr{M}=\mathscr{A} / \operatorname{Aut}(P)$ is simply connected and has a natural structure of a smooth compact manifold. For any $A \in \mathscr{A}^{\prime}$, there is a natural isomorphism

$$
T_{[A]} \mathscr{M} \simeq H_{A}^{1}(\Sigma)
$$

sending the tangent vector at $t=0$ of a smooth curve $t \mapsto A_{t} \in \mathscr{A}^{\prime}$ with $A_{0}=A$ to the cohomology class of $\left.\frac{d}{d t}\right|_{t=0} A_{t} \in \Omega^{1}(\Sigma, \mathfrak{s u}(m))$. Furthermore, for any $\alpha, \beta \in$ $\Omega^{1}(\Sigma, \mathfrak{s u}(m))$ with $d_{A} \alpha=d_{A} \beta=0$, the formula

$$
\omega^{\mathscr{M}}([\alpha],[\beta])=-\frac{m}{4 \pi^{2}} \int_{\Sigma} \operatorname{Tr}(\alpha \wedge \beta)
$$


defines a symplectic form $\omega^{\mathscr{M}}$ on $\mathscr{M}$ via (5.23).

Note that the symplectic form (5.24) is $m$ times the canonical symplectic form of $\mathscr{M}$, as defined in [10, p.587].

Let $f \in \operatorname{Diff}^{+}(\Sigma, D)$ be an orientation preserving diffeomorphism of $\Sigma$ fixing $D$ pointwise, inducing a diffeomorphism

$$
\begin{aligned}
\varphi: \mathscr{M} & \longrightarrow \mathscr{M} \\
{[A] } & \longmapsto\left[f^{*} A\right]
\end{aligned}
$$

preserving (5.24). The following result is classical and can be deduced from [21, §4.2] and $[45, \S 3.3, \S 4.3]$.

Proposition 5.10. There is a natural Hermitian line bundle with connection $\left(L, h^{L}, \nabla^{L}\right)$ over $\left(\mathscr{M}, \omega^{\mathscr{M}}\right)$ whose curvature satisfies the prequantization condition (1.1), and a natural lift $\varphi^{L}$ of (5.25) to L preserving metric and connection.

Note that this line bundle is the $m$-th power of the Chern-Simons bundle of [45, $\S 3.3]$, which is only an orbifold line bundle in general. The lift $\varphi^{L}$ is the one acting trivially on the second summand of $\mathscr{A} \times \mathbb{C}$ in the description of this line bundle as a quotient given at the end of $[45, \S 3.3]$.

Recall that the group Diff ${ }_{0}(\Sigma)$ of diffeomorphisms isotopic to the identity acts naturally on the space $\mathscr{J}_{\Sigma}$ of complex structures on $\Sigma$. As explained for example in [7, $\S 3-4]$, the Teichmüller space $\mathscr{T}_{\Sigma}:=\mathscr{J}_{\Sigma} / \operatorname{Diff}_{0}(\Sigma)$ has a natural structure of a complex manifold. Following [33, §2], we identify complex structures on $\Sigma$ with Hodge-star operators on $\Omega^{1}(\Sigma)$, and write $* \in \mathscr{J}_{\Sigma}$. Recall that $\alpha \in \Omega^{1}(\Sigma, \mathfrak{s u}(m))$ satisfying $d_{A} \alpha=* d_{A} \alpha=0$ is called harmonic. Recalling Definition 5.4, the following result follows from [33, (2.6), (2.15)] and [12, §2].

Proposition 5.11. The first projection $\pi: \mathscr{T}_{\Sigma} \times \mathscr{M} \rightarrow \mathscr{T}_{\Sigma}$ has a structure of a rigid holomorphic tautological fibration, with associated line bundle $\left(L, h^{L}, \nabla^{L}\right)$ as above. For any $* \in \mathscr{J}_{\Sigma}$ and $A \in \mathscr{A}^{\prime}$, the associated relative complex structure $J \in \operatorname{End}(T \mathscr{M})$ is defined over $([*],[A]) \in \mathscr{T}_{\Sigma} \times \mathscr{M}$ via (5.23) and Hodge theory by

$$
J \alpha=-* \alpha,
$$

where $\alpha \in \Omega^{1}(\Sigma, \mathfrak{s u}(m))$ is the unique harmonic representative of $[\alpha] \in H_{A}^{1}(\Sigma)$.

The associated relative Riemannian metric on the relative tangent bundle $T \mathscr{M}$ over $([*],[A]) \in \mathscr{T}_{\Sigma} \times \mathscr{M}$ is then given via (5.23) and Hodge theory by

$$
g^{T \mathscr{M}}([\alpha],[\beta])=\frac{m}{4 \pi^{2}} \int_{\Sigma}\langle\alpha, \beta\rangle_{T^{*} \Sigma} d v_{\Sigma}
$$

where $\alpha, \beta \in \Omega^{1}(\Sigma, \mathfrak{s u}(m))$ are the unique harmonic representative of $[\alpha],[\beta] \in H_{A}^{1}(\Sigma)$, for $\langle\cdot, \cdot\rangle_{T^{*} \Sigma}$ and $d v_{\Sigma}$ induced by any Riemannian metric $g^{T \Sigma}$ associated with $* \in \mathscr{J}_{\Sigma}$, which we take to be the associated hyperbolic metric. 
For any $A \in \mathscr{A}^{\prime}$ and $* \in \mathscr{J}_{\Sigma}$, let $d_{A}=: \partial_{A}+\bar{\partial}_{A}$ be the decomposition of $d_{A}$ with respect to the splitting (2.4) of $(T \Sigma, *)$. Consider the universal family of Riemann surfaces over $\mathscr{T}_{\Sigma}$ as in [7, Th.5.6], and give it the structure of a holomorphic prequantized fibration as in $[38, \S 5.1]$. By the construction of [18, Th.1.9], we can consider the holomorphic determinant line bundle $\operatorname{det}\left(\bar{\partial}_{A}\right)$ of the induced family of $\bar{\partial}$-operators over $\mathscr{M} \times \mathscr{T}_{\Sigma}$. The following theorem is a consequence of the curvature formula of [18, Th.1.9]. It follows from $[33, \S 2]$ and the computations of $[12,(4.17)]$.

Proposition 5.12. The canonical bundle $K_{\mathscr{M}}$ over $\mathscr{M} \times \mathscr{T}_{\Sigma}$ is isomorphic to the dual of the determinant line bundle $\operatorname{det}\left(\bar{\partial}_{A}\right)$ considered above. Furthermore, the Chern curvature of its natural Hermitian metric $h^{K \mathscr{M}}$ induced by (5.27) satisfies

$$
\frac{\sqrt{-1}}{2 \pi}\left[-R^{K \mathscr{M}}+\bar{\partial} \partial \rho\right]^{\mathscr{M}}=2 \omega^{\mathscr{M}},
$$

where $\rho \in \mathscr{C}^{\infty}\left(\mathscr{M} \times \mathscr{T}_{\Sigma}, \mathbb{R}\right)$ is the analytic torsion of the associated family of $\bar{\partial}$-operators induced by the hyperbolic metric associated with $* \in \mathscr{T}_{\Sigma}$.

The metric $h^{Q}:=e^{-\rho} h^{K_{\mathscr{M}}}$ on $K_{\mathscr{M}}$ is called the Quillen metric, and the Chern connection $\nabla^{Q}$ of $\left(K_{\mathscr{M}}, h^{Q}\right)$ satisfies

$$
\nabla^{Q}=\nabla^{K \mathscr{M}}-\partial \rho
$$

For $* \in \mathscr{J}_{\Sigma}$ and $f \in \operatorname{Diff}^{+}(\Sigma, D)$ as above, let $\gamma:[0,1] \rightarrow \mathscr{T}_{\Sigma}$ be a path joining $[*]$ to $\left[f^{*} *\right]$, and consider the pullback of the universal family of Riemann surfaces over $\mathscr{T}_{\Sigma}$ by $\gamma$. This induces a holomorphic prequantized fibration

$$
\pi_{f}: \Sigma_{f} \simeq \Sigma \times[0,1] /[(0, x) \sim(1, f(x))] \longrightarrow \mathbb{R} / \mathbb{Z},
$$

and we call $\Sigma_{f}$ the mapping torus of $f$. We write $(\Sigma \backslash D)_{f} \subset \Sigma_{f}$ for the mapping torus of $\left.f\right|_{\Sigma \backslash D}$.

By Propositions 5.9 to 5.12 , we can apply Theorem 5.5 with $k=2$ and consider the parallel transport with respect to the connection $\left\{\nabla^{p}\right\}_{p \in \mathbb{N}^{*}}$ of $(5.18)$ in the quantum bundle $\left\{\mathscr{H}_{p}\right\}_{p \in \mathbb{N}^{*}}$ associated with $\pi: \mathscr{T}_{\Sigma} \times \mathscr{M} \rightarrow \mathscr{T}_{\Sigma}$ along $\gamma:[0,1] \rightarrow \mathscr{T}_{\Sigma}$. This is precisely the canonical projectively flat connection of $[12, \S 4 . \mathrm{b}]$ on the Verlinde bundle. By Lemma 5.6, we are then precisely in the context of Section 4 , with $(X, \omega)=\left(\mathscr{M}, \omega^{\mathscr{M}}\right)$ and $\varphi: \mathscr{M} \rightarrow \mathscr{M}$ defined in (5.25).

Proof of Theorem 1.3 and Theorem 1.4. Let $\mathscr{A}_{f}$ be the space of flat $\mathrm{SU}(m)$-connections over $(\Sigma \backslash D)_{f}$ with holonomy around the boundary equal to $e^{\frac{2 \pi \sqrt{-1} d}{m}} \operatorname{Id}_{\mathbb{C}^{m}} \in \mathrm{SU}(m)$, and let $\mathscr{M}_{f}$ be the associated moduli space defined as above. As all $A \in \mathscr{A}$ are irreducible, the same is true for all $A_{f} \in \mathscr{A}_{f}$. By assumption, the analogue of Proposition 5.9 applies to $\mathscr{M}_{f}$, so that it is smooth and its tangent space at any $\left[A_{f}\right] \in \mathscr{M}_{f}$ identifies with the first cohomology group $H_{A_{f}}^{1}\left(\Sigma_{f}\right)$ of $\left(\Omega_{A_{f}}^{\bullet}\left(\Sigma_{f}\right), d_{A_{f}}\right)$ defined as above. Following [36, §5.2.1] and [28, §8.1], consider the map $r: \mathscr{M}_{f} \rightarrow \mathscr{M}$ defined by restriction over 
any fibre of (5.30), which satisfies $\operatorname{Im} r=\mathscr{M}^{\varphi}$ and $\# r^{-1}([A])=m$, where $\mathscr{M}^{\varphi}$ denotes the fixed point set of $\varphi: \mathscr{M} \rightarrow \mathscr{M}$ defined in (5.25).

For any $A_{f} \in \mathscr{A}_{f}$, let $A \in \mathscr{A}$ be the restriction of $A_{f}$ to any fibre of (5.30), so that $r\left(\left[A_{f}\right]\right)=[A]$. Recall as in [33, p. 359] that the irreducibility of $A \in \mathscr{A}$ implies that $H_{A}^{0}(\Sigma)=H_{A}^{2}(\Sigma)=\{0\}$. Let $d t$ be the canonical volume form of $\mathbb{R} / \mathbb{Z}$. Following $[3$, $(4.2)$ ], we have the following long exact sequence in cohomology

$$
0 \longrightarrow H_{A_{\varphi}}^{1}\left(\Sigma_{\varphi}\right) \stackrel{r^{*}}{\longrightarrow} H_{A}^{1}(\Sigma) \stackrel{\mathrm{Id}-f^{*}}{\longrightarrow} H_{A}^{1}(\Sigma) \stackrel{\bullet \wedge \pi_{f}^{*} d t}{\longrightarrow} H_{A_{\varphi}}^{2}\left(\Sigma_{\varphi}\right) \longrightarrow 0,
$$

where the first map is induced by restriction on any fibre of (5.30), and thus identifies with the differential of $r: \mathscr{M}_{f} \rightarrow \mathscr{M}$ via (5.23). This shows that $r: \mathscr{M}_{f} \rightarrow \mathscr{M}$ is a smooth immersion, and thus a smooth $m$-covering on its image $\operatorname{Im} r=\mathscr{M}^{\varphi}$, which is smooth as well. Furthermore, the exactness of (5.31) together with (5.23) and (5.25) implies that $T \mathscr{M}^{\varphi}=\operatorname{Ker}\left(\operatorname{Id}_{T \mathscr{M}}-d \varphi\right)$. Thus the fixed point set of $\varphi$ is non-degenerate, and we can apply Theorem 4.3 to this situation. The asymptotic expansion (1.10) is then an immediate consequence of Theorem 4.3, where the locally constant value of $\varphi^{L}$ restricted to $\mathscr{M}^{\varphi}$ has been computed in [28, Prop.8.4] to be equal to (1.9). The densities over $\mathscr{M}_{f}$ are obtained by pullback by $r: \mathscr{M}_{f} \rightarrow \mathscr{M}^{\varphi}$.

Let us now show (1.11). Restrict the prequantized fibration $\pi: \mathscr{T}_{\Sigma} \times \mathscr{M} \rightarrow \mathscr{T}_{\Sigma}$ over the path $\gamma:[0,1] \rightarrow \mathscr{T}_{\Sigma}$ as in Section 2.3, and let $[A] \in \mathscr{M}$ be fixed by $\varphi$. Then under the assumptions of Lemma 5.2, with $E=\mathbb{C}$ and $\tau^{E, \sigma}$ determined by Lemma 5.6, we get over any connected component of $\mathscr{M}^{\varphi}$ of dimension $2 d$ and for some coherent choices of square roots,

$$
\nu_{0}=(-1)^{\frac{n-d}{2}}\left(\varphi^{K \mathscr{M}} \tau^{Q,-1}\right)^{\frac{1}{2}}\left|\operatorname{det}_{N}\left(\operatorname{Id}_{N}-\left.d \varphi\right|_{N}\right)\right|^{-\frac{1}{2}}|d v|_{T \mathscr{M} / N}
$$

where $\tau^{Q}$ is the parallel transport on $K^{\mathscr{M}}$ with respect to $(5.29)$ and $|d v|_{T \mathscr{M} / N}$ is the density over $\mathscr{M}^{\varphi}$ satisfying (4.25). Following [5, Cor.4.3], the Reidemeister torsion $\tau_{\Sigma_{\varphi}}\left(\operatorname{ad} A_{\varphi}\right)$ of ad $A_{\varphi}$ over $\Sigma_{\varphi}$ is equal to the torsion of the complex (5.31), which is identified with an element of $\operatorname{det} H_{A_{\varphi}}^{1}\left(\Sigma_{\varphi}\right)^{-2}$ via Poincaré duality. Thus the absolute value $\left|\tau_{\Sigma_{\varphi}}\left(\operatorname{ad} A_{\varphi}\right)\right|^{\frac{1}{2}}$ can be identified with a density over $\mathscr{M}^{\varphi}$. Following [5, Th.5.6] and using the fact that $\mathrm{Id}-d \varphi$ preserves a transverse subbundle $N$ of $T \mathscr{M}^{\varphi}$ as in $[5$, Prop.5.4], we deduce from (4.25) and (5.31) that

$$
\left|\tau_{\Sigma_{\varphi}}\right|^{\frac{1}{2}}=\left|\operatorname{det}_{N}\left(\operatorname{Id}_{N}-\left.d \varphi\right|_{N}\right)\right|^{-\frac{1}{2}}|d v|_{T \mathscr{M} / N}
$$

Now let $d_{A}+d_{A}^{*}$ be the even signature operator on $\Omega_{A}^{\bullet}(\Sigma, \mathfrak{s u}(m))$, where $d_{A}^{*}$ is the dual of $d_{A}$ with respect to (5.27). By a construction of [16], we can consider the associated determinant line bundle over $\mathscr{T}_{\Sigma} \times \mathscr{M}$, equipped with the Bismut-Freed connection of [16, Def.1.17]. We then have the following lemma, which follows from an argument of $[8, \S 4,(4.12)]$ using Proposition 5.12 and [18].

Lemma 5.13. The square $K_{\mathscr{M}}^{2}$ of the relative canonical line bundle of $\pi: \mathscr{T}_{\Sigma} \times \mathscr{M} \rightarrow$ $\mathscr{T}_{\Sigma}$ is isomorphic to the determinant line bundle of the even signature operator over $\mathscr{T}_{\Sigma} \times \mathscr{M}$. Furthemore, this isomorphism sends the connection induced by $\nabla^{Q}$ on $K_{\mathscr{M}}^{2}$ to the Bismut-Freed connection. 
Then as explained in $[8, \S 4]$, the holonomy theorem of $[15$, Th.3.16] implies that the parallel transport with respect to the Bismut-Freed connection over the loop $(\gamma,[A])$ as above is given by $\exp \left(-\sqrt{-1} \pi \eta^{0}\left(A_{f}\right)\right)$, where $\eta^{0}\left(A_{f}\right)$ is the limit as $\varepsilon \rightarrow 0$ of the $\eta$-invariant of the odd signature operator $(-1)^{k}\left(d_{A_{f}} *_{\varepsilon}+*_{\varepsilon} d_{A_{f}}\right)$ acting on the odd forms $\oplus_{k=1}^{2} \Omega_{A_{f}}^{2 k-1}\left(\Sigma_{f}, \mathfrak{s u}(m)\right)$, where $*_{\varepsilon}$ is the Hodge-star operator of the metric $g^{T \Sigma} \oplus \varepsilon^{-2} g^{T \mathbb{R}}$ constructed via (2.2) over the fibration $\pi_{f}: \Sigma_{f} \rightarrow \mathbb{R} / \mathbb{Z}$. By Lemma 5.13, this shows that

$$
(-1)^{\frac{n-d}{2}}\left(\varphi^{K} \mathscr{M} \tau^{Q,-1}\right)^{\frac{1}{2}}=(\sqrt{-1})^{k} \exp \left(\frac{\sqrt{-1} \pi}{4} \eta^{0}\left(\operatorname{ad} A_{\varphi}\right)\right),
$$

with $k \in \mathbb{Z} / 4 \mathbb{Z}$ locally constant by continuity of the other terms. Then the integral over $\mathscr{M}_{f}$ descends to $m$ times an integral over $\mathscr{M}^{\varphi}$ via the $m$-covering $r: \mathscr{M}_{f} \rightarrow \mathscr{M}^{\varphi}$. This completes the proof of (1.11).

Let us finally show Theorem 1.4. Taking the expansion of the fractional power in $p \in$ $\mathbb{N}^{*}$, the associated asymptotic expansion as in (1.10) follows from Theorem 4.3 as above, and to compute the first coefficient (1.13), we can assume that $E=\pi^{*} \operatorname{det}\left(\bar{\partial}_{\Sigma}\right)^{-\frac{m^{2}-1}{2}}$. Using $[8, \S 4]$ and [18] as above, the analogue of Lemma 5.13 holds for $\operatorname{det}\left(\bar{\partial}_{\Sigma}\right)$ over $\mathscr{T}_{\Sigma}$ instead of $K_{\mathscr{M}}$, replacing $d_{A}$ by the usual exterior differential $d$ on $\Omega^{\bullet}(\Sigma, \mathbb{C})$. Thus the parallel transport in $E$ along $\gamma$ with respect to the connection induced by (5.29) is given by $(\sqrt{-1})^{k} \exp \left(-\frac{\sqrt{-1} \pi}{4}\left(m^{2}-1\right) \eta^{0}(0)\right)$ for some $k \in \mathbb{Z} / 4 \mathbb{Z}$, where $\eta^{0}(0)$ is the limit as $\varepsilon \rightarrow 0$ of the $\eta$-invariant of the usual odd signature operator $(-1)^{k}\left(d *_{\varepsilon}+*_{\varepsilon} d\right)$ acting on $\oplus_{k=1}^{2} \Omega^{2 k-1}\left(\Sigma_{f}, \mathbb{C}\right)$. By definition of the $\rho$-invariant in [11, Th.2.4] and using $\operatorname{dim} \mathfrak{s u}(m)=m^{2}-1$, we get

$$
\rho\left(\operatorname{ad} A_{\varphi}\right)=\eta^{0}\left(\operatorname{ad} A_{\varphi}\right)-\left(m^{2}-1\right) \eta^{0}(0) .
$$

This completes the proof of (1.13).

\section{References}

[1] J. E. Andersen, Asymptotic faithfulness of the quantum $S U(N)$ representations of the mapping class groups, Ann. of Math. 163 (2006), no. 2, 347-368.

[2] _ Hitchin's connection, Toeplitz operators, and symmetry invariant deformation quantization, Quantum Topol. 3 (2012), no. 3-4, 293-325.

[3] _ , The Witten-Reshetikhin-Turaev invariants of finite order mapping tori I, J. Reine Angew. Math. 681 (2013), 1-38.

[4] J. E. Andersen, N. L. Gammelgaard, and M. R. Lauridsen, Hitchin's connection in metaplectic quantization, Quantum Topol. 3 (2012), no. 3-4, 327-357.

[5] J. E. Andersen and B. Himpel, The Witten-Reshetikhin-Turaev invariants of finite order mapping tori II, Quantum Topol. 3 (2012), no. 3-4, 377-421.

[6] J. E. Andersen and W. E. Petersen, Asymptotic expansions of the WittenReshetikhin-Turaev Invariants of Mapping Tori I, ArXiv e-prints (2018), arxiv.org/abs/1803.09510v2. 
[7] E. Arbarello and M. Cornalba, Teichmüller space via Kuranishi families, Ann. Sc. Norm. Super. Pisa Cl. Sci. (5) 8 (2009), no. 1, 89-116.

[8] M. Atiyah, The logarithm of the Dedekind $\eta$-function, Math. Ann. 278 (1987), no. 1-4, 335-380.

[9] _ On framings of 3-manifolds, Topology 29 (1990), no. 1, 1-7.

[10] M. F. Atiyah and R. Bott, The Yang-Mills equations over Riemann surfaces, Philos. Trans. Roy. Soc. London Ser. A 308 (1983), no. 1505, 523-615.

[11] M. F. Atiyah, V. K. Patodi, and I. M. Singer, Spectral asymmetry and Riemannian geometry. II, Math. Proc. Cambridge Philos. Soc. 78 (1975), no. 3, 405-432.

[12] S. Axelrod, S. Della Pietra, and E. Witten, Geometric quantization of chern-simons gauge theory, J. Differential Geom. 33 (1991), no. 3, 787-902.

[13] N. Berline, E. Getzler, and M. Vergne, Heat kernels and Dirac operators, Grundlehren Text Editions, Springer-Verlag, Berlin, 2004, Corrected reprint of the 1992 original.

[14] J.-M. Bismut, The Atiyah-Singer index theorem for families of Dirac operators: two heat equation proofs, Invent. Math. 83 (1986), no. 1, 91-151.

[15] J.-M. Bismut and D. S. Freed, The analysis of elliptic families. I. Metrics and connections on determinant bundles, Comm. Math. Phys. 106 (1986), no. 1, 159176.

[16] - The analysis of elliptic families. II. Dirac operators, eta invariants, and the holonomy theorem, Comm. Math. Phys. 107 (1986), no. 1, 103-163.

[17] J.-M. Bismut, H. Gillet, and C. Soulé, Analytic torsion and holomorphic determinant bundles. II. Direct images and Bott-Chern forms, Comm. Math. Phys. 115 (1988), no. 1, 79-126.

[18] _ Analytic torsion and holomorphic determinant bundles. III. Quillen metrics on holomorphic determinants, Comm. Math. Phys. 115 (1988), no. 2, 301-351.

[19] J.-M. Bismut and S. Goette, Holomorphic equivariant analytic torsions, Geom. Funct. Anal. 10 (2000), no. 6, 1289-1422.

[20] J.-M. Bismut and K. Köhler, Higher analytic torsion forms for direct images and anomaly formulas, J. Algebraic Geom. 1 (1992), no. 4, 647-684.

[21] J.-M. Bismut and F. Labourie, Symplectic geometry and the Verlinde formulas, Surveys in differential geometry: differential geometry inspired by string theory, Surv. Differ. Geom., vol. 5, Int. Press, Boston, MA, 1999, pp. 97-311.

[22] J.-M. Bismut and E. Vasserot, The asymptotics of the Ray-Singer analytic torsion associated with high powers of a positive line bundle, Comm. Math. Phys. 125 (1989), no. 2, 355-367.

[23] M. Bordemann, E. Meinrenken, and M. Schlichenmaier, Toeplitz quantization of Kähler manifolds and $\mathrm{gl}(N), N \rightarrow \infty$ limits, Comm. Math. Phys. 165 (1994), no. 2, 281-296.

[24] L. Boutet de Monvel and V. Guillemin, The spectral theory of Toeplitz operators, Annals of Mathematics Studies, vol. 99, Princeton University Press, Princeton, NJ; University of Tokyo Press, Tokyo, 1981.

[25] L. Boutet de Monvel and J. Sjöstrand, Sur la singularité des noyaux de Bergman 
et de Szegö, (1976), 123-164. Astérisque, No. 34-35.

[26] L. Charles, Semi-classical properties of geometric quantization with metaplectic correction, Comm. Math. Phys. 270 (2007), no. 2, 445-480.

[27] _ A Lefschetz fixed point formula for symplectomorphisms, J. Geom. Phys. 60 (2010), no. 12, 1890-1902.

[28] _ Asymptotic properties of the quantum representations of the mapping class group, Trans. Amer. Math. Soc. 368 (2016), no. 10, 7507-7531.

[29] X. Dai, K. Liu, and X. Ma, On the asymptotic expansion of Bergman kernel, J. Differential Geom. 72 (2006), no. 1, 1-41.

[30] T. Foth and A. Uribe, The manifold of compatible almost complex structures and geometric quantization, Comm. Math. Phys. 274 (2007), no. 2, 357-379.

[31] W. M. Goldman, The symplectic nature of fundamental groups of surfaces, Adv. in Math. 54 (1984), no. 2, 200-225.

[32] V. Guillemin and A. Uribe, The Laplace operator on the $n$-th tensor power of a line bundle: eigenvalues which are uniformly bounded in n, Asymptotic Anal. 1 (1988), no. 2, 105-113.

[33] N. J. Hitchin, Flat connections and geometric quantization, Comm. Math. Phys. 131 (1990), no. 2, 347-380.

[34] L. Ioos, Geometric quantization of Hamiltonian flows and the Gutzwiller trace formula, Lett. Math. Phys. 110 (2020), 1585-1621.

[35] L. Ioos, W. Lu, X. Ma, and G. Marinescu, Berezin-toeplitz quantization for eigenstates of the bochner-laplacian on symplectic manifolds, J. Geom. Anal. 30 (2020), no. 3, 2615-2646.

[36] L. C. Jeffrey, Chern-Simons-Witten invariants of lens spaces and torus bundles, and the semiclassical approximation, Comm. Math. Phys. 147 (1992), no. 3, 563604.

[37] W. D. Kirwin and S. Wu, Geometric quantization, parallel transport and the Fourier transform, Comm. Math. Phys. 266 (2006), no. 3, 577-594.

[38] S. Klevtsov, X. Ma, G. Marinescu, and P. Wiegmann, Quantum Hall effect and Quillen metric, Comm. Math. Phys. 349 (2017), no. 3, 819-855.

[39] W. Lu, X. Ma, and G. Marinescu, Donaldson's Q-operators for symplectic manifolds, Sci. China Math. 60 (2017), no. 6, 1047-1056.

[40] X. Ma and G. Marinescu, The $\operatorname{Spin}^{c}$ Dirac operator on high tensor powers of a line bundle, Math. Z. 240 (2002), no. 3, 651-664.

[41] _ Holomorphic Morse inequalities and Bergman kernels, Progress in Mathematics, vol. 254, Birkhäuser Verlag, Basel, 2007.

[42] _ Generalized Bergman kernels on symplectic manifolds, Adv. Math. 217 (2008), no. 4, 1756-1815.

[43] - Toeplitz operators on symplectic manifolds, J. Geom. Anal. 18 (2008), no. 2, 565-611.

[44] X. Ma and W. Zhang, Superconnection and family Bergman kernels, C. R. Math. Acad. Sci. Paris 344 (2007), no. 1, 41-44.

[45] E. Meinrenken and C. Woodward, Canonical bundles for Hamiltonian loop group 
manifolds, Pacific J. Math. 198 (2001), no. 2, 477-487.

[46] P. Scheinost and M. Schottenloher, Metaplectic quantization of the moduli spaces of flat and parabolic bundles, J. Reine Angew. Math. 466 (1995), 145-219.

[47] M. Schlichenmaier, Deformation quantization of compact Kähler manifolds by Berezin-Toeplitz quantization, Conférence Moshé Flato 1999, Vol. II (Dijon), Math. Phys. Stud., vol. 22, Kluwer Acad. Publ., Dordrecht, 2000, pp. 289-306.

[48] G. Segal, The definition of conformal field theory, Topology, geometry and quantum field theory, London Math. Soc. Lecture Note Ser., vol. 308, Cambridge Univ. Press, Cambridge, 2004, pp. 421-577.

[49] G. M. Tuynman, Quantization: towards a comparison between methods, J. Math. Phys. 28 (1987), no. 12, 2829-2840.

[50] E. Witten, Quantum field theory and the Jones polynomial, Comm. Math. Phys. 121 (1989), no. 3, 351-399.

[51] S. Zelditch, Index and dynamics of quantized contact transformations, Ann. Inst. Fourier (Grenoble) 47 (1997), no. 1, 305-363.

Tel Aviv University - School of Mathematical Sciences, Ramat Aviv, Tel Aviv 69978, Israël

E-mail adress: louisioos@mail.tau.ac.il 